\title{
The Iron Yield of Normal Type II Supernovae
}

\author{
Ó. Rodríguez, ${ }^{1 \star}$ N. Meza, ${ }^{2}$ J. Pineda-García ${ }^{3}$ and M. Ramirez ${ }^{3}$ \\ ${ }^{1}$ School of Physics and Astronomy, Tel Aviv University, Tel Aviv 69978, Israel \\ ${ }^{2}$ Department of Physics, University of California, 1 Shields Avenue, Davis, CA 95616, USA \\ ${ }^{3}$ Departamento de Ciencias Fisicas, Universidad Andres Bello, Avda. Republica 252, Santiago, Chile
}

Accepted XXX. Received YYY; in original form ZZZ

\begin{abstract}
We present ${ }^{56} \mathrm{Ni}$ mass estimates for 110 normal Type II supernovae (SNe II), computed here from their luminosity in the radioactive tail. This sample consists of $\mathrm{SNe}$ from the literature, with at least three photometric measurements in a single optical band within 95-320 d since explosion. To convert apparent magnitudes to bolometric ones, we compute bolometric corrections (BCs) using $15 \mathrm{SNe}$ in our sample having optical and near-IR photometry, along with three sets of SN II atmosphere models to account for the unobserved flux. We find that the $I$ - and $i$-band are best suited to estimate luminosities through the $\mathrm{BC}$ technique. The ${ }^{56} \mathrm{Ni}$ mass distribution of our SN sample has a minimum and maximum of 0.005 and $0.177 \mathrm{M}_{\odot}$, respectively, and a selection-bias-corrected average of $0.037 \pm 0.005 \mathrm{M}_{\odot}$. Using the latter value together with iron isotope ratios of two sets of core-collapse (CC) nucleosynthesis models, we calculate a mean iron yield of $0.040 \pm 0.005 \mathrm{M}_{\odot}$ for normal SNe II. Combining this result with recent mean ${ }^{56} \mathrm{Ni}$ mass measurements for other CC SN subtypes, we estimate a mean iron yield $<0.068 \mathrm{M}_{\odot}$ for $\mathrm{CC} \mathrm{SNe}$, where the contribution of normal $\mathrm{SNe}$ II is $>36$ per cent. We also find that the empirical relation between ${ }^{56} \mathrm{Ni}$ mass and steepness parameter $(S)$ is poorly suited to measure the ${ }^{56} \mathrm{Ni}$ mass of normal SNe II. Instead, we present a correlation between ${ }^{56} \mathrm{Ni}$ mass, $S$, and absolute magnitude at $50 \mathrm{~d}$ since explosion. The latter allows to measure ${ }^{56} \mathrm{Ni}$ masses of normal SNe II with a precision around 30 per cent.
\end{abstract}

Key words: supernovae: general - nuclear reactions, nucleosynthesis, abundances

\section{INTRODUCTION}

Supernovae (SNe) explosions are important astrophysical objects for a wide range of research fields. Among them we mention their use to measure distances and cosmological parameters, their connection with stellar evolution, and their contribution to the energetics and chemical enrichment of the interstellar medium. Indeed, regarding the latter, SNe synthesize the bulk of all the mass in the Universe residing in elements from oxygen to the iron group. Therefore, to understand chemical evolution, it is critical to determine how much elements are produced by every kind of SN: Type Ia and core-collapse (CC) SNe (see Hoeflich 2017 and Burrows \& Vartanyan 2021 for current reviews of their explosion mechanisms). In the case of $\mathrm{CC} \mathrm{SNe,} \mathrm{almost} \mathrm{all}$ $\alpha$-elements have been produced in those explosions, while their contribution to the cosmic iron budget is comparable to that of SNe Ia (e.g. Maoz \& Graur 2017).

$\mathrm{CC} \mathrm{SNe}$ are grouped into two classes: H-rich envelope $\mathrm{SNe}$, historically known as Type II SNe (SNe II,

* E-mail: olrodrig@gmail.com
Minkowski 1941), and stripped-envelope (SE) SNe. The latter group includes $\mathrm{H}$-poor Type IIb and H-free Type Ib, Ic, and broad-line Ic (Ic-BL) SNe (see Gal-Yam 2017 for a current review of the SN classification). Among SNe II, some events are grouped into subtypes based on spectral and photometric characteristics: those showing narrow $\mathrm{H}$ emission lines in the spectra, indicative of ejecta-circumstellar material interaction (SNe IIn; Schlegel 1990) ${ }^{1}$, and those having long-rising light curves similar to SN 1987A (Hamuy et al. 1988; Taddia et al. 2016); while a few SNe II are recognized as having peculiar characteristics (e.g. OGLE14-073, Terreran et al. 2017; iPTF14hls, Arcavi et al. 2017; ASASSN-15nx, Bose et al. 2018; DES16C3cje, Gutiérrez et al. 2020; SN 2018ivc, Bostroem et al. 2020). For the rest of SNe II (about 90 per cent, e.g. Shivvers et al. 2017), in order not to use the same name of the class that contains other SN II subtypes and peculiar events, we will refer as normal

${ }^{1}$ In this group we also include SNe IIn/II and LLEV SNe II, described in Rodríguez et al. (2020). 
$\mathrm{SNe}$ II. The latter are found to form a continuum group ${ }^{2}$ (e.g. Anderson et al. 2014; Sanders et al. 2015; Valenti et al. 2016; Gutiérrez et al. 2017b; de Jaeger et al. 2019), where the photometric and spectroscopic diversity depends mainly on the amount of $\mathrm{H}$ in the envelope at the moment of the explosion, the synthesized ${ }^{56} \mathrm{Ni}$ mass $\left(M_{56} \mathrm{Ni}\right)$, and the explosion energy (e.g. Gutiérrez et al. 2017b).

Progenitors of normal SNe II have been directly detected on pre-explosion images. They correspond to red supergiant (RSG) stars with zero-age main-sequence (ZAMS) mass, MzAMs, in the range $8-18 \mathrm{M}_{\odot}$ (e.g. Smartt 2009, 2015). In the case of SE SNe, evidence points toward progenitors with $M_{\text {ZAMS }}$ similar to those of normal SNe II but evolving in binary systems, and some cases with a more massive and isolated progenitor (e.g. Anderson 2019 and references therein). The ${ }^{56} \mathrm{Ni}$ mass produced by CC SNe depends on the explosion properties and the core structure of the progenitor (e.g. Suwa et al. 2019). Therefore, $M_{56}{ }_{\mathrm{Ni}}$ estimates are important to contrast the progenitor scenarios and explosion mechanisms of different CC SNe. The mean ${ }^{56} \mathrm{Ni}$ mass $\left(\left\langle M_{56} \mathrm{Ni}\right\rangle\right)$ of normal SNe II is lower than that of SE SNe (e.g. Anderson 2019; Meza \& Anderson 2020). On the other hand, normal SNe II account for around 60 per cent of all CC SNe in a volume-limited sample (e.g. Shivvers et al. 2017). The latter makes normal SNe II significant contributors to the ${ }^{56} \mathrm{Ni}$ and iron budget of CC SNe (e.g. see Section 4.4).

Normal SNe II are characterized by having an optically thick photosphere during the first 60-120 d after the explosion (e.g. Anderson et al. 2014; Faran et al. 2014a; Sanders et al. 2015; de Jaeger et al. 2019). In this so-called photospheric phase, the $V$-band absolute magnitudes $\left(M_{V}\right)$ range between around -14.5 and $-18.5 \mathrm{mag}$ (e.g. Anderson et al. 2014; de Jaeger et al. 2019). In particular, normal SNe II having $M_{V} \gtrsim-15.5$ mag are referred as sub-luminous SNe II (e.g. Pastorello et al. 2004; Spiro et al. 2014), while those having $M_{V} \lesssim-17 \mathrm{mag}$ are referred as moderately-luminous SNe II (e.g. Inserra et al. 2013). The aforementioned phase is also characterized by a period of 30-70 d where the $V$-band magnitude remains nearly constant or declines linearly with time (e.g. Anderson et al. 2014). During this period, called plateau phase, light curves are powered by $\mathrm{H}$ recombination. Then, the brightness decreases by around $1.0-3.5 \mathrm{mag}$ in a lapse of about $15-30 \mathrm{~d}$, indicating that all $\mathrm{H}$ has recombined. After this transition phase, the luminosity starts to decrease exponentially with time. In this phase, the energy sources are the $\gamma$ rays and positrons produced by the radioactive decay of the unstable cobalt isotope ${ }^{56} \mathrm{Co}$ (daughter of the unstable nickel isotope ${ }^{56} \mathrm{Ni}$ ) into the stable iron isotope ${ }^{56} \mathrm{Fe}$ (e.g. Weaver \& Woosley 1980). Therefore the luminosity in this phase, called radioactive tail, is a good estimate of the ${ }^{56} \mathrm{Ni}$ mass ejected in the explosion.

The luminosity is given by the inverse-square law of light and the bolometric flux. The latter can be computed through the direct integration technique (e.g. Lusk \& Baron 2017). In this method, the available $x$-band magnitudes are converted to monochromatic fluxes $\left(\bar{f}_{x}\right)$ and associated

2 Some authors, however, suggest a separation into distinct groups (e.g. Arcavi et al. 2012; Faran et al. 2014b). to $x$-band effective wavelengths $\left(\bar{\lambda}_{x}\right)$. The set of $\left(\bar{\lambda}_{x}, \bar{f}_{x}\right)$ points defines the photometric spectral energy distribution (pSED) which, integrated over wavelength, provides the quasi-bolometric flux. For normal SNe II in the radioactive tail, the quasi-bolometric flux in the wavelength range $0.46-2.16 \mu \mathrm{m}$ typically accounts for 90 per cent of the bolometric flux (e.g. see Section 3.3). To estimate the unobserved flux, some authors extrapolate fluxes assuming a Planck function (e.g. Hamuy 2001; Pejcha \& Prieto 2015a), while others do not include the unobserved flux in the bolometric flux (e.g. Bersten \& Hamuy 2009; Maguire et al. 2010). In practice, the application of the direct integration technique is limited because the low number of normal SNe II with IR photometry during the radioactive tail.

An alternative method to compute bolometric fluxes for SNe without IR photometry is the bolometric correction (BC) technique (e.g. Lusk \& Baron 2017). In this method, the magnitude in a given band $\left(m_{x}\right)$ is related to the bolometric magnitude $\left(m_{\mathrm{bol}}\right)$ through $m_{\mathrm{bol}}=m_{x}+\mathrm{BC}_{x}$. Here, $\mathrm{BC}_{x}$ is calibrated using $\mathrm{SNe}$ with $m_{\text {bol }}$ computed with the direct integration technique. Based on the BVIJHK photometry of the normal SN II 1999em and the long-rising SN 1987A, Hamuy (2001) reported a constant $\mathrm{BC}_{V}$ for $\mathrm{SNe}$ II in the radioactive tail. Similar constant $\mathrm{BC}_{V}$ values were later reported by other authors (e.g. Bersten \& Hamuy 2009; Maguire et al. 2010; Pejcha \& Prieto 2015a).

The two main weaknesses in the $\mathrm{BC}_{V}$ values reported in previous works are: (1) not accounting for the unobserved flux or assuming a Planck function to estimate it, and (2) the low number of $\mathrm{SNe}$ used to compute $\mathrm{BC}_{V}$. At present, thanks to the development of non-local thermodynamic equilibrium (non-LTE) radiative transfer codes (e.g. Dessart \& Hillier 2011; Jerkstrand et al. 2011), it is possible to estimate the flux outside the optical/near-IR range for normal SNe II through theoretical spectral models. From the observational side, the number of normal SNe II observed during the radioactive tail with optical and near-IR filters had increased over time. Therefore, it is possible to improve the $\mathrm{BC}$ determination with the current available data.

The goal of this work is to estimate the mean iron yield $\left(\bar{y}_{\mathrm{Fe}}\right)$ of normal SNe II. For this, we use all normal SNe II in the literature with useful photometry during the radioactive tail. To improve the determination of the radioactive tail luminosity through the $\mathrm{BC}$ technique, we also aim to enhance the $\mathrm{BC}$ calibration. The paper is organized as follows. In Section 2 we outline the relevant information on the data we use. The methodology to compute BCs, ${ }^{56} \mathrm{Ni}$ and iron masses is described in Section 3. In Section 4 we present new BC calibrations, the $M_{56} \mathrm{Ni}$ distribution of our SN sample, the $\left\langle M_{56}{ }_{\mathrm{Ni}}\right\rangle$ and $\bar{y}_{\mathrm{Fe}}$ values for normal SNe II, and a new method to estimate $M_{56} \mathrm{Ni}$. Comparisons with previous works, discussions about systematics, and future improvements are in Section 5. Finally, our conclusions are summarized in Section 6.

\section{DATA SET}

\subsection{Supernova sample}

For this work we select normal SNe II from the literature, having photometry in the radioactive tail (1) in at least one 
of the following bands: Johnson-Kron-Cousins VRI or Sloan $r i$; (2) in the range 95-320 d since the explosion, corresponding to the time range where our $\mathrm{BC}$ calibrations are valid (Section 4.2.2); and (3) with at least 3 photometric epochs in order to detect possible $\gamma$-ray leakage from the ejecta (Section 3.4). The final sample of 110 normal SNe II fulfilling our selection criteria is listed in Table L1. This includes the SN name (Column 1), the host galaxy name (Column 2), the Galactic colour excess $E_{B-V}^{\mathrm{G}}$ (Column 3), the heliocentric SN redshift $z_{\text {helio }}^{\mathrm{SN}}$ (Column 4), the distance modulus (Column 5, see Section 3.6.1), the host galaxy colour excess $E_{B-V}^{\mathrm{h}}(\mathrm{Col}-$ umn 6, see Section 3.6.2), the explosion epoch $t_{0}$ (Column 7, see Section 3.7) and the references for the photometry (Column 8). Among the SNe in our set, 15 have $B V(r) R(i) I J H K$ photometry in the radioactive tail. We use the latter sample to compute bolometric fluxes and BCs. Galactic colour excesses are taken from Schlafly \& Finkbeiner (2011) (except for SN 2002hh, see Section 3.6.2), which have associated a random error of 16 per cent (Schlegel et al. 1998). Throughout this work, for our Galaxy and host galaxies, we assume the extinction curve given by Fitzpatrick (1999) with $R_{V}=3.1$ (except for SN 2002hh, see Section 3.6.2).

\subsection{Theoretical models}

We use SN II atmosphere models to compute the contribution of the flux at $\lambda<\bar{\lambda}_{B}$ and $\lambda>\bar{\lambda}_{K}$ to the bolometric one. We also employ $\mathrm{CC}$ nucleosynthesis models to estimate the contribution of iron stable isotopes other than ${ }^{56} \mathrm{Fe}$ to the total ejected iron mass $\left(M_{\mathrm{Fe}}\right)$. Among the available models from the literature, we select those using progenitors of $M_{\text {ZAMS }} \leq 18 \mathrm{M}_{\odot}$ to be consistent with observations of normal SNe II (e.g. Smartt 2009, 2015). Those models were exploded with energies $\sim 10^{50}-10^{51} \mathrm{erg}$, corresponding to the typical range for normal SNe II (e.g. Förster et al. 2018; Morozova et al. 2018). The ${ }^{56} \mathrm{Ni}$ masses of the selected models range between 0.003 and $0.12 \mathrm{M}_{\odot}$ which is consistent with observations of normal SNe II (e.g. Müller et al. 2017).

\subsubsection{Atmosphere models}

We use the SN II atmosphere model sets given by Dessart et al. (2013, hereafter D13), Jerkstrand et al. (2014, hereafter J14), and Lisakov et al. (2017, hereafter L17). Those models were generated evolving ZAMS stars with $M_{\text {ZAMS }} \geq 12 \mathrm{M}_{\odot}$ until the RSG stage before the CC. The explosions were simulated using a piston, while the spectra were computed through 1D non-LTE radiative transfer codes. In particular, L17 used the same methodology as D13 but with different progenitors and explosion energies.

The selected D13 set consists on six models $\left(8-11\right.$ spectra each) with $M_{\mathrm{ZAMS}}=15 \mathrm{M}_{\odot} \quad$ and $M_{56 \mathrm{Ni}}=0.036-0.121 \mathrm{M}_{\odot}$. The selected J14 set consists on three models (3-5 spectra each) with $M_{\mathrm{ZAMS}}$ of 12 , 15 , and $19 \mathrm{M}_{\odot}$, and $M_{56 \mathrm{Ni}}=0.062 \mathrm{M}_{\odot}$. The selected $\mathrm{L} 17$ set consists on seven models (9-10 spectra each) with $M_{\mathrm{ZAMS}}=12 \mathrm{M}_{\odot}$, and $M_{56 \mathrm{Ni}}=0.007-0.010 \mathrm{M}_{\odot}$.

\subsubsection{Nucleosynthesis yields}

We use the 1D CC nucleosynthesis yields presented in Kobayashi et al. (2006, hereafter K06) and Sukhbold et al. (2016, hereafter S16), which are updated versions of the works of Nomoto et al. (1997) and Woosley \& Weaver (1995), respectively. The selected K06 models were calibrated to be consistent with observations of normal SNe II, where the yields are estimated using a mass cut such that $M_{\mathrm{Fe}} \sim 0.07 \mathrm{M}_{\odot}$. The $\mathrm{S} 16$ models were calibrated to be consistent with with the Crab SN (for $M_{\mathrm{ZAMS}} \leq 12 \mathrm{M}_{\odot}$ ) and SN 1987A (for $M_{\text {ZAMS }}>12 \mathrm{M}_{\odot}$ ), while the yields were estimated using the "special trajectory" to represent the mass cut (see S16 for more details).

The selected K06 set consists on 12 models with $M_{\text {ZAMS }}$ of 13,15 , and $18 \mathrm{M}_{\odot}$, and $M_{56} \mathrm{Ni}=0.07-0.09 \mathrm{M}_{\odot}$. The selected S16 set consist on 13 models with $M_{\mathrm{ZAMS}}=9-12 \mathrm{M}_{\odot}$ and $M_{56 \mathrm{Ni}}=0.003-0.03 \mathrm{M}_{\odot}$, and 89 models with $M_{\mathrm{ZAMS}}$ between 12 and $18 \mathrm{M}_{\odot}$ and $M_{56}{ }_{\mathrm{Ni}}=0.05-0.09 \mathrm{M}_{\odot}$.

\section{METHODOLOGY}

\subsection{Light-curve interpolation}

To compute bolometric fluxes and BCs, we first need to evaluate the $B V(r) R(i) I J H K$ photometry of the $15 \mathrm{SNe}$ in the calibration sample at the same set of epochs $\left\{t_{i}\right\}$. To determine these epochs, for each band we select the epochs covered simultaneously by the photometry in the other bands. Then, we adopt the epochs of the band with less observations as $\left\{t_{i}\right\}$. If the rest of bands do not have photometry at the $t_{i}$ epochs, then we interpolate them using the ALR code $^{3}$ (Rodríguez et al. 2019). The ALR performs loess nonparametric regressions (Cleveland et al. 1992) to the input photometry, taking into account observed and intrinsic errors, along with the presence of possible outliers. If the ALR is not able to perform a loess fit (e.g. only few data points are available), then the ALR just performs a linear interpolation between points. In the case of SN 1995ad, we extrapolate the $B I$ photometry to the epoch of the JHK photometry using a straight-line fit. Fig. 1 shows the result of this process.

\subsection{Quasi-bolometric correction}

In the $\mathrm{BC}$ calibration sample, only 6 out of $15 \mathrm{SNe}$ have $\mathrm{ri}$ photometry. Therefore, we only use the BVRIJHK photometry in order to compute quasi-bolometric fluxes with an homogeneous data set. We construct pSEDs and compute quasi-bolometric fluxes $F_{i}^{B V R I J H K}$ (in $\mathrm{erg} \mathrm{s}^{-1} \mathrm{~cm}^{-2}$ ) using the prescription provided in Appendix B. We define the $x$ band quasi $\mathrm{BC}(\mathrm{qBC})$ as

$\mathrm{qBC}_{x, i}=-2.5 \log \left(F_{i}^{\text {BVRIJHK }}\right)-m_{x, i}^{\mathrm{cor}}$,

where

$m_{x, i}^{\mathrm{cor}}=m_{x, i}-R_{\bar{\lambda}_{x}}\left(E_{B-V}^{\mathrm{G}}+E_{B-V}^{\mathrm{h}}\right)$,

being $m_{x, i}$ the $x$-band magnitude at epoch $t_{i}$, and $R_{\bar{\lambda}_{x}}$ the total-to-selective extinction ratio for $\bar{\lambda}_{x}$, listed in Table A1.

${ }^{3}$ https://github.com/olrodrig/ALR 


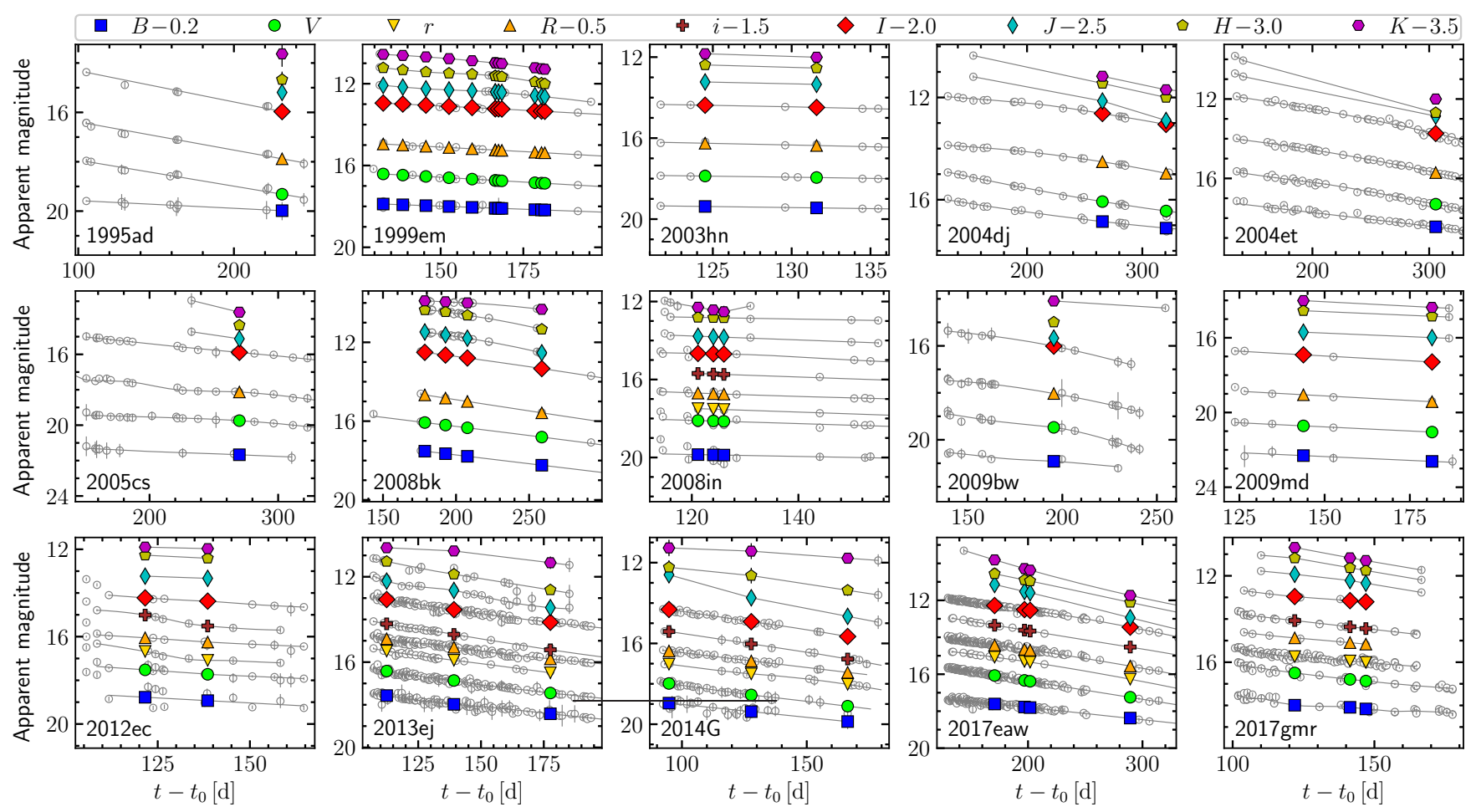

Figure 1. Light curves of the $15 \mathrm{SNe}$ in the BC calibration sample. Colour-filled symbols correspond to the interpolated photometry, where bands and magnitude shifts with respect to the original values are indicated in the legend. Grey empty circles correspond to the photometry from the literature, and grey lines are the light-curve fits.

\subsection{Bolometric correction and luminosity}

The flux from a pSED defined in a wavelength range $\lambda_{1}-\lambda_{2}$ is only an approximation of the real flux computed integrating the SED in the same wavelength range, $F_{i}^{\lambda_{1}-\lambda_{2}}$. In our case, to quantify the relative difference between $F_{i}^{B V R I J H K}$ and $F_{i}^{\bar{\lambda}_{B}-\bar{\lambda}_{K}}$, we compute $\alpha_{i}$ such that

$F_{i}^{\bar{\lambda}_{B}-\bar{\lambda}_{K}}=\left(1+\alpha_{i}\right) F_{i}^{B V R I J H K}$.

For this task we use the D13, J14, and L17 spectral models. To obtain $F_{i}^{B V R I J H K}$ from models, we first compute their synthetic magnitudes (see Appendix A) and then we compute $F_{i}^{B V R I J H K}$ with the recipe given in Appendix B.

Fig. 2 shows the $\alpha_{i}$ values computed with the three model sets. Since we do not find any correlation with colour indices, in the figure we plot $\alpha_{i}$ as a function of the time since explosion. For the D13, J14, and L17 models, the mean $\alpha$ values and their sample standard deviation $(\hat{\sigma})$ errors are $-3.2 \pm 1.8,4.6 \pm 3.0$, and $1.1 \pm 1.4$ per cent, respectively. There is a difference of at least $2.6 \hat{\sigma}$ between the mean $\alpha$ values from D13 and J14 models. Based on late-time optical spectra of 38 normal SNe II, Silverman et al. (2017) found that the J14 models fit better to the observations than the D13 ones. This evidence favour the scenario where $F_{i}^{\bar{\lambda}_{B}-\bar{\lambda}_{K}}$ is $\sim 5$ per cent greater than $F_{i}^{B V R I J H K}$ instead of $\sim 3$ per cent lower. To be conservative, we adopt the average of the mean $\alpha$ values of the three models, i.e., $\alpha=0.8 \pm 3.9$ per cent $(1 \hat{\sigma}$ error). This value is consistent within $\pm 1 \hat{\sigma}$ with the results obtained for the three model sets.

To compute the bolometric flux $F^{\text {bol }}$, we have to correct
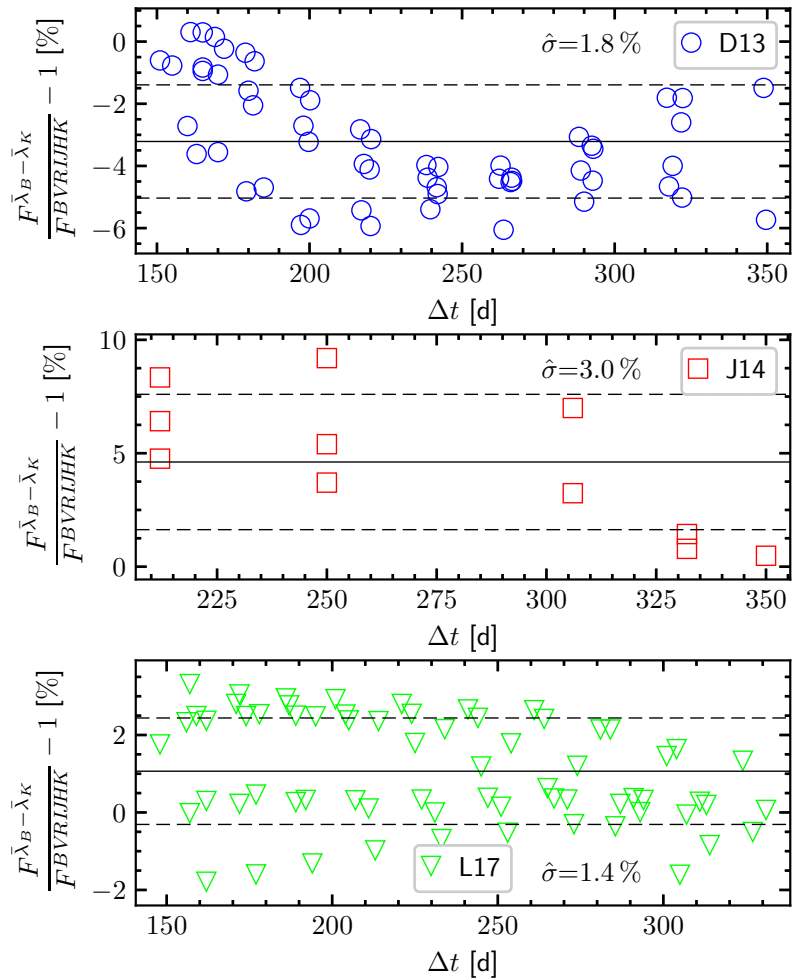

Figure 2. Relative differences between the flux computed with the pSED and the SED using the D13 (top panel), J14 (middle panel), and L17 (bottom panel) models. Solid and dashed lines correspond to mean values and $\pm 1 \hat{\sigma}$ limits, respectively. 
Table 1. Parameters for the flux corrections.

\begin{tabular}{lcrccc}
\hline Correction & Colour & $a_{0}(\%)$ & $a_{1}(\%)$ & $a_{2}(\%)$ & $\hat{\sigma}(\%)$ \\
\hline$\alpha$ & - & 0.80 & - & - & 3.90 \\
$c^{\lambda<\bar{\lambda}_{B}}$ & $B-V$ & -3.11 & 4.30 & 6.72 & 0.59 \\
$c^{\bar{\lambda}_{K}-2.48 \mu \mathrm{m}}$ & $V-K$ & 0.32 & 0.55 & - & 0.23 \\
$c^{2.48-3.0 \mu \mathrm{m}}$ & $V-K$ & 0.31 & 1.09 & -0.14 & 0.19 \\
$c^{\lambda>3 \mu \mathrm{m}}$ & $V-K$ & 1.87 & 0.52 & - & 0.24 \\
$c^{\lambda>\bar{\lambda}_{K}}$ & $V-K$ & 2.50 & 2.16 & -0.14 & 0.38 \\
\hline Note: $c^{\lambda>\lambda_{K}}=c^{\lambda}{ }^{-2.48 \mu \mathrm{m}}+c^{2.48-3.0 \mu \mathrm{m}}+c^{\lambda>3 \mu \mathrm{m}}$.
\end{tabular}

$F^{\bar{\lambda}_{B}-\bar{\lambda}_{K}}$ for the unobserved flux. In our case,

$F_{i}^{\mathrm{bol}}=F_{i}^{\bar{\lambda}_{B}-\bar{\lambda}_{K}}+F_{i}^{\lambda<\bar{\lambda}_{B}}+F_{i}^{\lambda>\bar{\lambda}_{K}}$,

where $F_{i}^{\lambda<\bar{\lambda}_{B}}$ and $F_{i}^{\lambda>\bar{\lambda}_{K}}$ are the unobserved fluxes at wavelengths below $\bar{\lambda}_{B}$ and beyond $\bar{\lambda}_{K}$, respectively.

For the unobserved flux below $\bar{\lambda}_{B}$, we write

$F_{i}^{\lambda<\bar{\lambda}_{B}}=F_{i}^{\bar{\lambda}_{B}-\bar{\lambda}_{K}} c_{i}^{\lambda<\bar{\lambda}_{B}}$,

where

$c_{i}^{\lambda<\bar{\lambda}_{B}}=F_{i}^{0.1 \mu \mathrm{m}-\bar{\lambda}_{B}} / F_{i}^{\bar{\lambda}_{B}-\bar{\lambda}_{K}}$

is the flux correction relative to $F_{i}^{\bar{\lambda}_{B}-\bar{\lambda}_{K}}$. We choose $0.1 \mu \mathrm{m}$ as lower $\lambda$ value because it is the minimum wavelength in common for the three model sets. This value is also low enough to consider the flux negligible at shorter wavelengths.

Fig. 3(a) shows the $c_{i}^{\lambda<\bar{\lambda}_{B}}$ values as a function of $B-V$. As visible in the figure, there is a correlation between both quantities. The flux correction is lower than 2 per cent for $B-V>1.7$ and can be greater than 10 per cent for $B-V<0.9$. Within the colour range in common between the D13, J14, and L17 models $(1.0 \leq B-V \leq 1.45)$, we see that values for the three model sets are in good agreement. We parametrize the dependence of $c_{i}^{\lambda<\bar{\lambda}_{B}}$ on $B-V$ as

$c_{i}^{\lambda<\bar{\lambda}_{B}}=\sum_{j=0}^{2} \frac{a_{j}}{(B-V)_{i}^{j}}$,

where the quadratic order was determined using the model selection described in Appendix C. Table 1 lists the fit parameters along with the $\hat{\sigma}$ around the fit, which covers the colour range $0.79 \leq B-V \leq 2.02$. Among the $B-V$ colours of the $\mathrm{SNe}$ in our $\mathrm{BC}$ calibration set (marked as magenta ticks in the figure), four are below the lower limit (two of SN 2014G, one of SN 1995ad, and one of SN 2004dj). In order to prevent a misestimation on $c_{i}^{\lambda<\bar{\lambda}_{B}}$ due to extrapolations, for $B-V$ colours bluer than 0.79 mag we adopt the $c^{\lambda<\bar{\lambda}_{B}}$ correction for $B-V=0.79$.

For the unobserved flux at $\lambda>\bar{\lambda}_{K}$ we use

$F_{i}^{\lambda>\bar{\lambda}_{K}}=F_{i}^{\bar{\lambda}_{B}-\bar{\lambda}_{K}} c_{i}^{\lambda>\bar{\lambda}_{K}}$,

where, since the model sets do not cover the same wavelength range, we write

$c_{i}^{\lambda>\bar{\lambda}_{K}}=c_{i}^{\bar{\lambda}_{K}-2.48 \mu \mathrm{m}}+c_{i}^{2.48-3.0 \mu \mathrm{m}}+c_{i}^{\lambda>3 \mu \mathrm{m}}$,

being

$c_{i}^{\bar{\lambda}_{K}-2.48 \mu \mathrm{m}}=F_{i}^{\bar{\lambda}_{K}-2.48 \mu \mathrm{m}} / F_{i}^{\bar{\lambda}_{B}-\bar{\lambda}_{K}}$,

$c_{i}^{2.48-3.0 \mu \mathrm{m}}=F_{i}^{2.48-3.0 \mu \mathrm{m}} / F_{i}^{\bar{\lambda}_{B}-\bar{\lambda}_{K}}$,
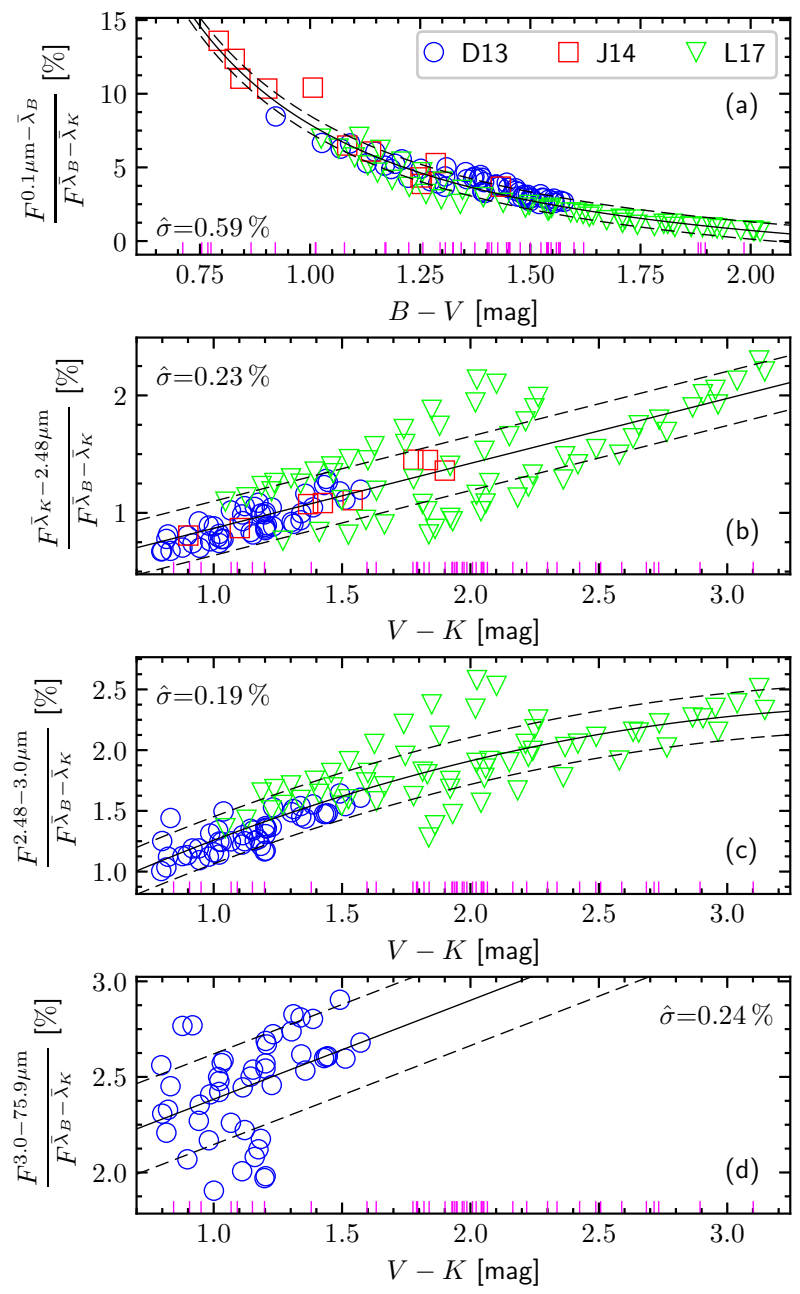

Figure 3. Corrections to account for the unobserved flux in the ranges $0.1 \mu \mathrm{m}-\bar{\lambda}_{B}$ (a), $\bar{\lambda}_{K}-2.48 \mu \mathrm{m}$ (b), 2.48-3.0 $\mu \mathrm{m}$ (c), and 3.0-75.9 $\mu \mathrm{m}(\mathrm{d})$, relative to the flux in the range $\bar{\lambda}_{B}-\bar{\lambda}_{K}$, using the D13, J14, and L17 models. Magenta ticks mark the colours of the $\mathrm{SNe}$ in the $\mathrm{BC}$ calibration set. Solid lines indicate the fits to the models, while dashed lines are the $\pm 1 \hat{\sigma}$ limits around the fit.

and

$c_{i}^{\lambda>3 \mu \mathrm{m}}=F_{i}^{3.0-75.9 \mu \mathrm{m}} / F_{i}^{\bar{\lambda}_{B}-\bar{\lambda}_{K}}$.

Here, the values 2.48 and $3.0 \mu \mathrm{m}$ correspond to the maximum $\lambda$ in common for the $\{\mathrm{D} 13, \mathrm{~J} 14, \mathrm{~L} 17\}$ and $\{\mathrm{D} 13, \mathrm{~L} 17\}$ model sets, respectively, while $75.9 \mu \mathrm{m}$ is the maximum $\lambda$ for the D13 models.

Figs. 3(b) and 3(c) show $c_{i}^{\bar{\lambda}_{K}-2.48 \mu \mathrm{m}}$ and $c_{i}^{2.48-3.0 \mu \mathrm{m}}$, respectively, as a function of $V-K$. Those flux corrections, as expected, are greater for red colours than for blue ones. We express the dependence of $c_{i}^{\bar{\lambda}_{K}-2.48 \mu \mathrm{m}}$ and $c_{i}^{2.48-3.0 \mu \mathrm{m}}$ on $V-K$ through polynomials, i.e.,

$c_{i}^{\lambda_{1}-\lambda_{2}}=\sum_{j=0}^{\mathcal{O}_{\lambda_{1}, \lambda_{2}}} a_{j}(V-K)_{i}^{j}$,

being the orders $\mathcal{O}_{\lambda_{1}, \lambda_{2}}$ determined with the model selection described in Appendix C. The fit parameter values are summarized in Table 1. As in the case of $c_{i}^{\lambda<\bar{\lambda}_{B}}$, we find a good agreement between models within the ranges in common.

Fig. 3(d) shows the $c_{i}^{\lambda>3 \mu \mathrm{m}}$ values as a function of 
$V-K$, where only the D13 models provide spectral information for $\lambda>3 \mu \mathrm{m}$. In this case the best fit is a straightline, whose parameters are reported in Table 1 . Since the D13 models do not cover all the $V-K$ colours of the SNe in the $\mathrm{BC}$ calibration set, the straight-line fit could introduce errors due to extrapolation. However, we find that $c_{i}^{\lambda>3 \mu \mathrm{m}}$ at $V-K=3.1$ (the reddest colour in the BC calibration set) is only 1 per cent (in value) greater than the correction for the reddest colour in the D13 models $(V-K=1.57)$. Therefore, we adopt the linear parametrization of $c_{i}^{\lambda>3 \mu \mathrm{m}}$ for all the $V-K$ colour range $(0.80 \leq V-K \leq 3.15)$.

Based on equation (9), $c_{i}^{\lambda>\bar{\lambda}_{K}}$ is a polynomial given by equation (13), where the coefficients $a_{j}$ are the sum of those of $c_{i}^{\bar{\lambda}_{K}-2.48 \mu \mathrm{m}}, c_{i}^{2.48-3.0 \mu \mathrm{m}}$, and $c_{i}^{\lambda>3 \mu \mathrm{m}}$. Parameters for $c_{i}^{\lambda>\bar{\lambda}_{K}}$ are given in Table 1 .

Once $\alpha, c_{i}^{\lambda<\bar{\lambda}_{B}}$, and $c_{i}^{\lambda>\bar{\lambda}_{K}}$ are determined, we define the apparent bolometric magnitude and the $x$-band BC as

$m_{\mathrm{bol}, i}=-2.5 \log \left(F_{i}^{B V R I J H K}\right)-\kappa_{i}$

and

$\mathrm{BC}_{x, i}=m_{\mathrm{bol}, i}-m_{x, i}^{\mathrm{cor}}=\mathrm{qBC}_{x, i}-\kappa_{i}$,

respectively. The model-based correction, $\kappa_{i}$, is given by

$\kappa_{i}=2.5 \log (1+\alpha)+2.5 \log \left(1+c_{i}^{\lambda<\bar{\lambda}_{B}}+c_{i}^{\lambda>\bar{\lambda}_{K}}\right)$,

where the error in $\kappa_{i}$ is dominated by the error in $\alpha$ (see Table 1). For the SNe in the BC calibration set, the modelbased correction ranges between $0.09 \mathrm{mag}$ (SN 2005cs) and $0.22 \mathrm{mag}$ (SN 2014G), with a median of $0.11 \mathrm{mag}$. Therefore, during the radioactive tail, the observed $F^{B V R I J H K}$ typically corresponds to 90 per cent of the bolometric flux.

Once $\mathrm{BC}_{x}$ values are calibrated with observations (section 4.2), luminosities ( $L$, in $10^{43} \mathrm{erg} \mathrm{s}^{-1}$ ) can be estimated through the $\mathrm{BC}$ technique, given by

$\log L_{i}=\left(\mu-\mathrm{BC}_{x, i}-m_{x, i}^{\mathrm{cor}}\right) / 2.5-2.922$,

where the constant provides the conversion from magnitude to cgs units.

\section{$3.4 \quad{ }^{56} \mathrm{Ni}$ Mass}

During the radioactive tail, the energy sources powering the ejecta are the $\gamma$-rays and positrons produced in the radioactive decay of ${ }^{56} \mathrm{Co}$ into ${ }^{56} \mathrm{Fe}$. The latter deposit energy in the ejecta at a rate $Q_{\text {dep }}$. Using equations (10)-(12) of Wygoda et al. (2019), and assuming that the deposited energy is immediately emitted, we can write the relation between $Q_{\text {dep }}\left(\right.$ in $10^{43} \mathrm{erg} \mathrm{s}^{-1}$ ) and $M_{56 \mathrm{Ni}}\left(\right.$ in $\mathrm{M}_{\odot}$ ) as

$\log Q_{\mathrm{dep}, i}=\log M_{56_{\mathrm{Ni}}}-0.39\left[\frac{\Delta t_{i}}{100 \mathrm{~d}}\right]+0.154+D_{i}$.

Here, $\Delta t_{i}=\left(t_{i}-t_{0}\right) /\left(1+z_{\text {helio }}^{\mathrm{SN}}\right)$ is the time since explosion in the $\mathrm{SN}$ rest frame, and

$D_{i}=\log \left(0.97 f_{\mathrm{dep}, i}+0.03\right)$,

where $f_{\text {dep }}$ is the $\gamma$-ray deposition function, which describes the fraction of the generated $\gamma$-ray energy deposited in the ejecta. Knowing the deposition function, $\log M_{56 \mathrm{Ni}}$ can be inferred by equating equations (17) and (18).

If all the $\gamma$-ray energy is deposited in the ejecta, then

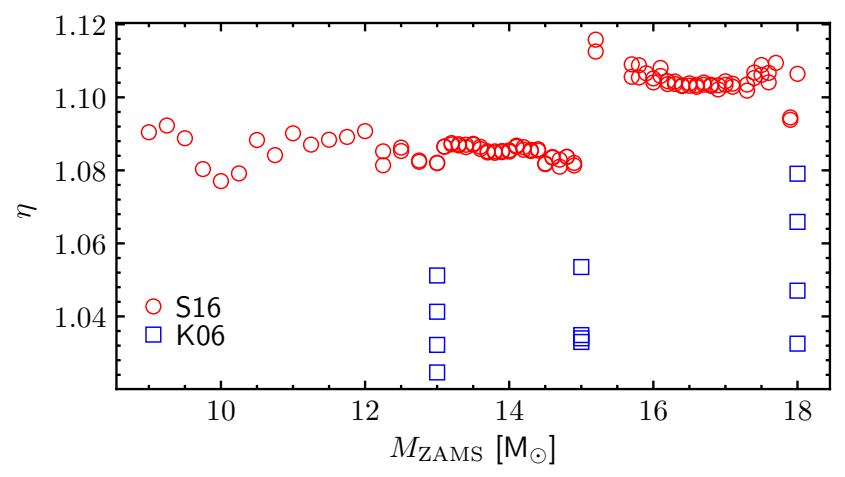

Figure 4. $M_{\mathrm{Fe}} / M_{56} \mathrm{Fe}$ against $M_{\mathrm{ZAMS}}$. Blue squares and red circles correspond to K06 and S16 models, respectively.

$f_{\mathrm{dep}, i}=1\left(D_{i}=0\right)$, otherwise $f_{\mathrm{dep}, i}<1 \quad\left(D_{i}<0\right)$. In the first case, we expect the $\log M_{56_{\mathrm{Ni}}}$ estimates (one for each $\log L_{i}$ measurement) to be consistent with a constant value. In the second case, the $\log M_{56_{\mathrm{Ni}}}$ estimates decrease with time as the ejecta becomes less able to thermalize $\gamma$-rays (Section 4.3.1), which makes it necessary to correct for $f_{\text {dep }}$. For the latter we adopt the model of Jeffery (1999):

$f_{\mathrm{dep}, i}=1-\exp \left[-\left(T_{0} / \Delta t_{i}\right)^{2}\right]$,

where $T_{0}$ is a characteristic time-scale (in d) that represents the $\gamma$-ray escape time. This parameter is estimated such that the $\log M_{56_{\mathrm{Ni}}}$ estimates are consistent with a constant value.

To detect possible $\gamma$-ray leakage from the ejecta, we need at least three photometric points as we have to infer $T_{0}$ and $\log M_{56 \mathrm{Ni}}$. The detailed recipe to compute $\log M_{56 \mathrm{Ni}}$ and check if it is necessary to correct for $f_{\text {dep }}$ is provided in Appendix $\mathrm{D}^{4}$. In order to properly convert $\log M_{56_{\mathrm{Ni}}}$ into $M_{56} \mathrm{Ni}$, we use the formalism provided in Appendix E.

\subsection{Iron mass}

The inferred $M_{56} \mathrm{Ni}$ provides a good estimate of the ejected ${ }^{56} \mathrm{Fe}$ mass $\left(M_{56} \mathrm{Fe}\right)$. The $M_{\mathrm{Fe}}$ value, however, is greater than $M_{56} \mathrm{Fe}$ since it is also composed of the stable isotopes ${ }^{54,57,58} \mathrm{Fe}$ (e.g. Curtis et al. 2019). Using the relation

$\eta=M_{\mathrm{Fe}} / M_{56} \mathrm{Ni}=1+M_{54,57,58} \mathrm{Fe} / M_{56} \mathrm{Fe}$

and the CC nucleosynthesis yield models of Iwamoto et al. (1999), Blanc \& Greggio (2008) computed $\eta=1.08$.

Fig. 4 shows the $\eta$ values obtained with the K06 and S16 models. Since we do not find any correlation with model parameters, in the figure we plot $\eta$ against $M_{\mathrm{ZAMS}}$. The $\eta$ values range between 1.03 and 1.11 , where the S16 values for $M_{\text {ZAMS }} \leq 15 \mathrm{M}_{\odot}$ are around 0.02 lower than those for $M_{\mathrm{ZAMS}}>15 \mathrm{M}_{\odot}$. The $\eta$ values computed with the K06 models are up to 0.06 lower than those from S16 models. Since neither model is preferred, to be conservative we adopt $\eta=1.07 \pm 0.04$ (the mid-point between 1.03 and 1.11, with the error being half the range). This value, consistent with that reported in Blanc \& Greggio (2008), indicates that the measured $M_{56 \mathrm{Ni}}$ accounts to about 93 per cent of $M_{\mathrm{Fe}}$.

4 The code implementing this algorithm (SNII_nickel) is available at https://github.com/olrodrig/SNII_nickel. 


\subsection{Host galaxy distance moduli and colour excesses}

The error on $\log M_{56} \mathrm{Ni}$ is mainly dominated by uncertainties on $\mu$ and $E_{B-V}^{\mathrm{h}}$ (e.g. Pejcha \& Prieto 2015b). To reduce their errors, we measure $\mu$ and $E_{B-V}^{\mathrm{h}}$ with various methods.

\subsubsection{Distance moduli}

To estimate $\mu$ for the SN host galaxies, we use distance moduli obtained with the Cepheids period-luminosity relation $\left(\mu_{\mathrm{CPL}}\right)$, the Tip of the Red Giant Branch method ( $\left.\mu_{\mathrm{TRGB}}\right)$, and the Tully-Fisher relation $\left(\mu_{\mathrm{TF}}\right)$. We compile $\mu_{\mathrm{CPL}}$ and $\mu_{\mathrm{TRGB}}$ values from the literature, and $\mu_{\mathrm{TF}}$ from the Extragalactic Distance Database ${ }^{5}$ (EDD, Tully et al. 2009). If a host galaxy does not have $\mu_{\mathrm{CPL}}$ nor $\mu_{\mathrm{TRGB}}$, then we include distances (1) computed with the Hubble-Lemaitre law $\left(D_{\text {HLL }}\right)$ using a local Hubble-Lemaître constant $\left(H_{0}\right)$ of $74.03 \pm 1.42 \mathrm{~km} \mathrm{~s}^{-1} \mathrm{Mpc}^{-1}$ (Riess et al. 2019) and including a velocity dispersion of $382 \mathrm{~km} \mathrm{~s}^{-1}$ to account for the effect of peculiar velocities over $D_{\mathrm{HLL}}$; and (2) from distancevelocity calculators based on smoothed velocity fields ( $\left.D_{\mathrm{SVF}}\right)$ given by Shaya et al. (2017) for $D_{\mathrm{SVF}}<38 \mathrm{Mpc}$ and Graziani et al. (2019) for $D_{\mathrm{SVF}}<200 \mathrm{Mpc}$. These calculators are available on the EDD website ${ }^{5}$ and described in Kourkchi et al. (2020). Since the latter do not provide distance uncertainties, we adopt the typical distance error of the neighbouring galaxies as a conservative estimate, or a 15 per cent error if the host galaxy is isolated (Ehsan Kourkchi, private communication). We convert $D_{\mathrm{HLL}}\left(D_{\mathrm{SVF}}\right)$ into $\mu_{\mathrm{HLL}}\left(\mu_{\mathrm{SVF}}\right)$ using the recipe provided in Appendix E.

Table L2 summarizes the aforementioned distance moduli. From this compilation, we adopt as $\mu$ the weighted average of $\mu_{\mathrm{CPL}}, \mu_{\mathrm{TRGB}}$, and $\mu_{\mathrm{TF}}$, if the first ones are available, otherwise we adopt the weighted average of $\mu_{\mathrm{TF}}, \mu_{\mathrm{HLL}}$, and $\mu_{\text {SVF. }}$. In the case of SN 2006my, whose host galaxy is within the Virgo Cluster, we include the distance modulus reported in Foster et al. (2014) based on the planetary nebula luminosity function. The $\mu$ values are in Column 5 of Table L1. The typical $\mu$ error is of $0.18 \mathrm{mag}$.

\subsubsection{Colour excesses}

To calculate $E_{B-V}^{\mathrm{h}}$ we use the following methods:

1. The colour-colour curve (C3) method (Rodríguez et al. 2014, 2019). This technique assumes that, during the plateau phase, all normal $\mathrm{SNe}$ II have similar linear $V-I$ versus $B-V$ C3s. Under this assumption, the $E_{B-V}^{\mathrm{h}}$ value of an SN can be inferred from the vertical displacement of its observed C3 with respect to a reddening-free C3 (for a graphical representation, see Fig. 3 of Rodríguez et al. 2014). Using the C3 method (Appendix F), implemented in the C3M code ${ }^{6}$, we measure the colour excesses $\left(E_{B-V}^{\mathrm{h}, \mathrm{C} 3}\right)$ of $71 \mathrm{SNe}$ in our set. Those values are reported in Column 2 of Table L3. The typical $E_{B-V}^{\mathrm{h}, \mathrm{C} 3}$ uncertainty is of $0.085 \mathrm{mag}$.

2. The colour method (e.g. Olivares E. et al. 2010). This technique assumes that all normal SNe II have the same intrinsic $V-I$ colour at the end of the plateau phase.
Olivares E. et al. (2010) defined this epoch as $30 \mathrm{~d}$ before the middle of the $V$-band transition phase $\left(t_{\mathrm{PT}, V}\right.$, see Section 4.5). The prescription provided by Olivares E. et al. (2010) to compute colour excesses can be written as

$$
E_{B-V}^{\mathrm{h}, V-I}=0.812\left[(V-I)_{-30}-0.656\right],
$$

$\sigma_{E_{B-V}^{\mathrm{h}, V-I}}=0.812 \sqrt{\sigma_{(V-I)_{-30}}^{2}+0.079^{2}}$.

Here, $(V-I)_{-30}$ is the $V-I$ colour measured at $t_{\mathrm{PT}, V}-30 \mathrm{~d}$ corrected for $E_{B-V}^{\mathrm{G}}$ and $K$-correction (e.g. Rodríguez et al. 2019). We compute $E_{B-V}^{\mathrm{h}, V-I}$ values for $59 \mathrm{SNe}$ in our sample. For SNe 2012aw and 2013am we adopt the values provided in the literature. Column 3 of Table L3 lists the $E_{B-V}^{\mathrm{h}, V-I}$ values, which have a typical uncertainty of 0.074 mag.

3. Spectrum-fitting method (e.g. Dessart et al. 2008; Olivares E. et al. 2010). This technique consists on inferring the colour excess $\left(E_{B-V}^{\mathrm{h}, \mathrm{spec}}\right)$ of an $\mathrm{SN}$ from the comparison between its spectra and those of reddening-corrected $\mathrm{SNe}$ or spectral models. We compile $E_{B-V}^{\mathrm{h} \text {,spec }}$ values from the literature for $22 \mathrm{SNe}$ in our set. We also compute $E_{B-V}^{\mathrm{h}, \mathrm{spec}}$ for $36 \mathrm{SNe}$ in our sample, using the prescription given in Appendix G. The $E_{B-V}^{\mathrm{h}, \mathrm{spec}}$ are collected in Column 4 of Table L3. The typical $E_{B-V}^{\mathrm{h}, \mathrm{spec}}$ uncertainty is of $0.091 \mathrm{mag}$.

Fig 5 shows residuals about the one-to-one relation between $E_{B-V}^{\mathrm{h}, \mathrm{C} 3}$ and $E_{B-V}^{\mathrm{h}, V-I}\left(r_{V-I}^{\mathrm{C} 3}\right.$, top panel $), E_{B-V}^{\mathrm{h}, V-I}$ and $E_{B-V}^{\mathrm{h}, \mathrm{spec}}\left(r_{\mathrm{spec}}^{V-I}\right.$, middle panel $)$, and between $E_{B-V}^{\mathrm{h}, \mathrm{spec}}$ and $E_{B-V}^{\mathrm{h}, \mathrm{C} 3}$ $\left(r_{\mathrm{C} 3}^{\mathrm{spec}}\right.$, bottom panel) for the $44 \mathrm{SNe}$ in our set having $E_{B-V}^{\mathrm{h}, \mathrm{C} 3}$, $E_{B-V}^{\mathrm{h}, V-I}$, and $E_{B-V}^{\mathrm{h}, \mathrm{spec}}$. For $r_{V-I}^{\mathrm{C} 3}$ we obtain a mean, $\hat{\sigma}$, and typical error of $0.02,0.09$, and $0.10 \mathrm{mag}$, respectively. For $r_{\mathrm{spec}}^{V-I}$ we compute a mean, $\hat{\sigma}$, and typical error of $0.00,0.14$, and $0.12 \mathrm{mag}$, respectively. For $r_{\mathrm{C} 3}^{\mathrm{spec}}$ we calculate a mean, $\hat{\sigma}$, and typical error of $-0.02,0.13$, and $0.12 \mathrm{mag}$, respectively. Since the $\hat{\sigma}$ values are quite similar to the typical residual errors, the observed dispersion is mainly due to colour excess errors. The mean offsets are statistically consistent with zero within $\pm 1.3 \hat{\sigma} / \sqrt{N}$. Therefore, we do not detect systematic differences between the colour excesses inferred with the three aforementioned methods. Based on the latter, for the $77 \mathrm{SNe}$ in our set having $E_{B-V}^{\mathrm{h}, \mathrm{C} 3}, E_{B-V}^{\mathrm{h}, V-I}$, and/or $E_{B-V}^{\mathrm{h}, \mathrm{spec}}$ estimates, we adopt the weighted mean of those values as $E_{B-V}^{\mathrm{h}}$. For SNe 1980K, 2006my, 2008gz, 2014cx, and 2017it we obtain negative $E_{B-V}^{\mathrm{h}}$ values (see Column 6 of Table L1). The values of the first four objects are consistent with zero within $1.3 \sigma$, while for SN 2017it the offset is of $-2.1 \sigma$. Although negative $E_{B-V}^{\mathrm{h}}$ values have no physical meaning, we keep those values as we do not have evidence to discard them.

For $\mathrm{SNe}$ in out sample without $E_{B-V}^{\mathrm{h}}$ estimates, we evaluate to use colour excesses inferred from the pseudoequivalent width of the host galaxy $\mathrm{NaID}$ absorption line $\left(\mathrm{pEW}_{\mathrm{NaID}}\right)$. We compile $\mathrm{pEW}_{\mathrm{NaID}}$ values from the literature $^{7}$ for $89 \mathrm{SNe}$ in our sample. With those values, we compute colour excesses $\left(E_{B-V}^{\mathrm{h}, \mathrm{NaID}}\right)$ using the relation of Poznanski et al. (2012), and adopting a relative $E_{B-V}$ error of 68 per cent (Phillips et al. 2013). The $\mathrm{pEW}$ NaID becomes insensitive to estimate the colour excess

5 http://edd.ifa.hawaii.edu/

6 https://github.com/olrodrig/C3M

7 If pEW NaID is not reported but $E_{B-V}^{\mathrm{h}}$ is, then we recover $\mathrm{pEW}_{\mathrm{NaID}}$ using the corresponding $E_{B-V}\left(\mathrm{pEW} \mathrm{NaID}_{\mathrm{N}}\right)$ calibration. 


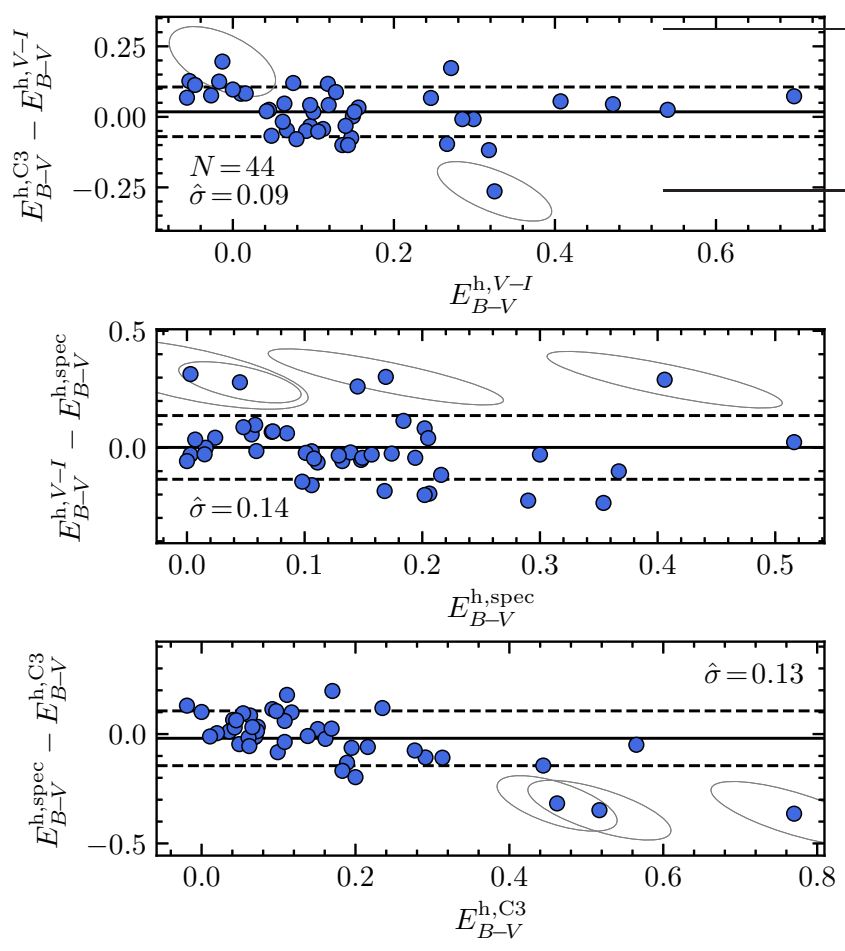

Figure 5. Residuals about the one-to-one relation between $E_{B-V}^{\mathrm{h}}$ values computed with the $\mathrm{C} 3$ method and the colour method (top panel), the colour method and the spectrum-fitting technique (middle panel), and the spectrum-fitting technique and the C3 method (bottom panel). Solid lines are mean values and dashed lines are $\pm 1 \hat{\sigma}$ limits. Ellipses are $1 \sigma$ confidence regions, which for clarity are drawn only for $\mathrm{SNe}$ outside $\pm 2 \hat{\sigma}$ from the mean.

for $\mathrm{pEW}$ NaID $>0.1 \mathrm{~nm}$ (e.g. Phillips et al. 2013), equivalent to $E_{B-V}>0.21 \pm 0.14 \mathrm{mag}$ in the Poznanski et al. (2012) relation. Therefore, we assume the latter lower limit for all SNe with $\mathrm{pEW}$ NaID greater than $0.1 \mathrm{~nm}$. The $E_{B-V}^{\mathrm{h}, \mathrm{NaID}}$ values are listed in Column 4 of Table L3.

Fig 6(a) shows $E_{B-V}^{\mathrm{h}, N a I D}$ against $E_{B-V}^{\mathrm{h}}(54 \mathrm{SNe})$. Fitting a straight line with a slope of unity, we measure an offset and $\hat{\sigma}$ value of -0.09 and $0.11 \mathrm{mag}$, respectively. The offset is equivalent to $-6.0 \hat{\sigma} / \sqrt{N}$, which means that the $E_{B-V}^{\mathrm{h}, \mathrm{NaID}}$ values are systematically lower than $E_{B-V}^{\mathrm{h}}$. Therefore, for the $24 \mathrm{SNe}$ in our sample without $E_{B-V}^{\mathrm{h}}$ estimates but with $E_{B-V}^{\mathrm{h}, \mathrm{NaID}}$ values we adopt $E_{B-V}^{\mathrm{h}}=E_{B-V}^{\mathrm{h}, \mathrm{NaID}}+0.09 \mathrm{mag}$, including in quadrature an error of $0.11 \mathrm{mag}$.

For the highly reddened SNe 2002hh and 2016ija (both without $E_{B-V}^{\mathrm{h}}$ and with $E_{B-V}^{\mathrm{h}, \text { NaID }}>0.21 \mathrm{mag}$ ) we adopt the $E_{B-V}^{\mathrm{h}}$ values reported by Pozzo et al. (2006) and Tartaglia et al. (2018), respectively. In the case of SN 2002hh, the colour excess has two components: $E_{B-V}=1.065 \mathrm{mag}$ (which includes $E_{B-V}^{\mathrm{G}}$ ) with $R_{V}=3.1$, and $E_{B-V}=1.545 \pm 0.182 \mathrm{mag}$ with $R_{V}=1.1$. For simplicity in the forthcoming analyses, we consider the first component as $E_{B-V}^{\mathrm{G}}$ and the second one as $E_{B-V}^{\mathrm{h}}$.

Fig. 6(b) shows the histogram of $E_{B-V}^{\mathrm{h}}$. To identify extreme values in the $E_{B-V}^{\mathrm{h}}$ distribution, we use the Chauvenet (1863) criterion. We find that SNe 2002hh, 2009hd, and 2016ija have $E_{B-V}^{\mathrm{h}}$ values greater than the Chauvenet upper rejection limit $\left(E_{B-V}^{\mathrm{h}}=1 \mathrm{mag}\right)$, so we consider them as outliers. The $E_{B-V}^{\mathrm{h}}$ distribution (removing the extreme values)
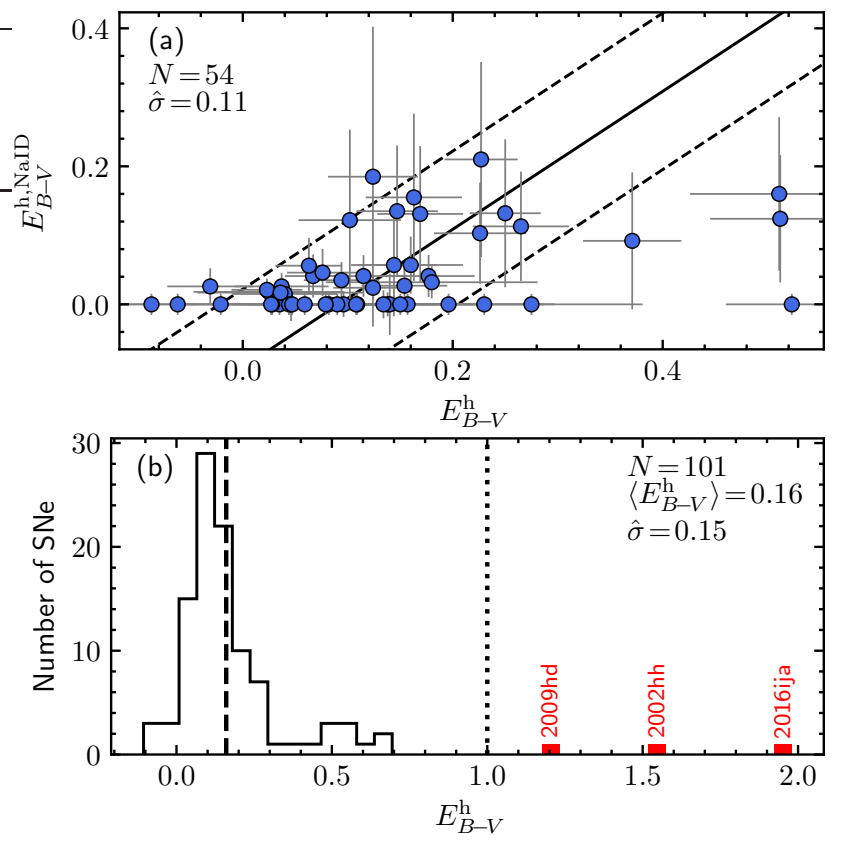

Figure 6. Panel (a): colour excesses computed from $\mathrm{pEW}$ NaID versus the $E_{B-V}^{\mathrm{h}}$ values adopted in this work. The solid line is a straight line fit with a slope of unity, and dashed lines are the $\pm 1 \hat{\sigma}$ limits around the fit. Error bars are $1 \sigma$ errors. Panel (b): histogram of $E_{B-V}^{\mathrm{h}}$. The dotted line is the Chauvenet upper rejection limit $\left(E_{B-V}^{\mathrm{h}}=1 \mathrm{mag}\right)$. The dashed line indicates the mean $E_{B-V}^{\mathrm{h}}$ of the 101 SNe with $E_{B-V}^{\mathrm{h}}<1 \mathrm{mag}$. Red bins are outliers.

has a mean and $\hat{\sigma}$ of 0.16 and $0.15 \mathrm{mag}$, respectively. For the six SNe in our set without $E_{B-V}^{\mathrm{h}}$ (SNe 2004eg, PTF11go, PTF11htj, PTF11izt, PTF12grj, and LSQ13dpa) we adopt the mean and $\hat{\sigma}$ of the latter distribution as $E_{B-V}^{\mathrm{h}}$ and its error, respectively.

The adopted $E_{B-V}^{\mathrm{h}}$ values are in Column 6 of Table L1. The typical $E_{B-V}^{\mathrm{h}}$ error is of $0.08 \mathrm{mag}$.

\subsection{Explosion epochs}

The SN explosion epoch is typically estimated as the midpoint between the last non-detection $t_{\mathrm{ln}}$ and the first SN detection $t_{\mathrm{fd}}$. In order to improve the $t_{0}$ estimates for the SNe in our set, we use the SNII_ETOS code ${ }^{8}$ (Rodríguez et al. 2019). The latter computes $t_{0}$ given a set of optical spectra as input and a uniform prior on $t_{0}$ provided by $t_{\mathrm{ln}}$ and $t_{\mathrm{fd}}$ (for more details, see Rodríguez et al. 2019). If SNII_ETOS is not able to compute $t_{0}$ for an SN, then we adopt the midpoint between $t_{\mathrm{ln}}$ and $t_{\mathrm{fd}}$ if $t_{\mathrm{fd}}-t_{\mathrm{ln}}<20 \mathrm{~d}$, otherwise we use the $t_{0}$ value reported in the literature.

Table L4 lists the $t_{\mathrm{ln}}$ (Column 2), $t_{\mathrm{fd}}$ (Column 3), and the adopted $t_{0}$ (Column 4 ) values (also reported in Column 7 of Table L1) for our SN set. The typical $t_{0}$ error is of $3.8 \mathrm{~d}$.

\footnotetext{
8 https://github.com/olrodrig/SNII_ETOS
} 

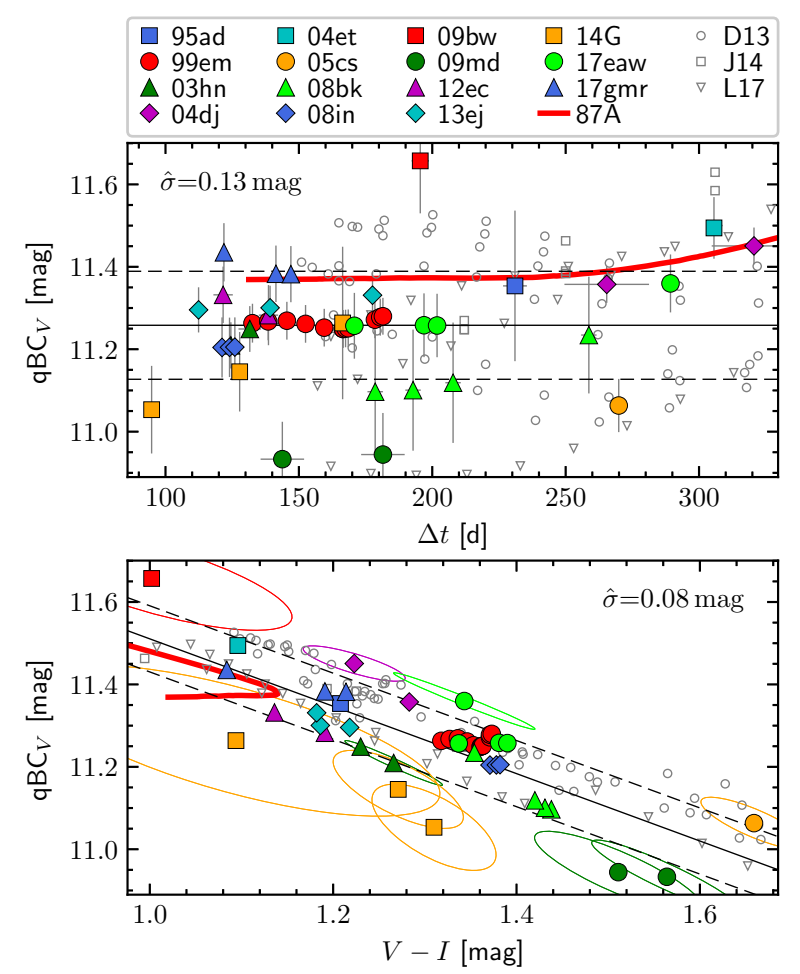

Figure 7. $\mathrm{qBC}_{V}$ values for the $\mathrm{SNe}$ in the $\mathrm{BC}$ calibration set (filled symbols), the D13, J14, and L17 models (empty symbols), and the long-rising SN 1987A (red thick line), as a function of time since explosion (top panel) and $V-I$ colour (bottom panel). Solid lines are the best polynomial fit to the observed data, while dashed lines indicate the $\pm 1 \hat{\sigma}$ limits around the fit. Error bars are $1 \sigma$ errors and ellipses indicate $1 \sigma$ confidence regions, which for clarity are drawn only for $\mathrm{SNe}$ outside the $\pm 1 \hat{\sigma}$ limits.

\section{ANALYSIS}

\subsection{Quasi-bolometric and bolometric corrections}

The top panel of Fig. 7 shows the $\mathrm{qBC}_{V}$ values for the $15 \mathrm{SNe}$ in the $\mathrm{BC}$ calibration set against the time since explosion. For comparison we include the D13, J14, and L17 models, along with the long-rising SN 1987A, which is typically used to estimate $\mathrm{BC}_{V}$ for normal SNe II (e.g. Hamuy 2001; Bersten \& Hamuy 2009; Maguire et al. 2010). In the figure we see that, except for SN $2014 \mathrm{G}$, the four SNe with three or more points at $\Delta t<250 \mathrm{~d}$ and a time baseline greater than $30 \mathrm{~d}$ (SNe 1999em, 2008bk, 2013ej, and 2017eaw) seems to be consistent with a constant $\mathrm{qBC}_{V}$ value, as in the case of SN 1987A. For SNe 2017eaw and 2008bk we notice that the $\mathrm{qBC}_{V}$ values at $\Delta t>250 \mathrm{~d}$ are $0.1-0.15 \mathrm{mag}$ greater than the values at $\Delta t<210 \mathrm{~d}$. This could be due to the effect of newly formed dust. We also notice differences in $\mathrm{qBC}_{V}$ of around $0.3-0.7 \mathrm{mag}$ between the sub-luminous SNe 2005cs, 2008bk, 2009md (e.g. Spiro et al. 2014) and the moderatelyluminous SNe 2004et and 2009bw (e.g. Inserra et al. 2013).

The bottom panel of Fig. 7 shows $\mathrm{qBC}_{V}$ versus $V-I$. We detect a correlation between both quantities, in the sense that the redder the $\mathrm{SN}$ the lower the $\mathrm{qBC}_{V}$. This correlation is also displayed by models (empty symbols), which are consistent with the linear fit to the observations (solid line) within $\pm 1 \hat{\sigma}$ (dashed lines). Since the errors in $E_{B-V}^{\mathrm{h}}, E_{B-V}^{\mathrm{G}}$, and $V$-band photometry affect the $\mathrm{qBC}_{V}$ and $V-I$ values,
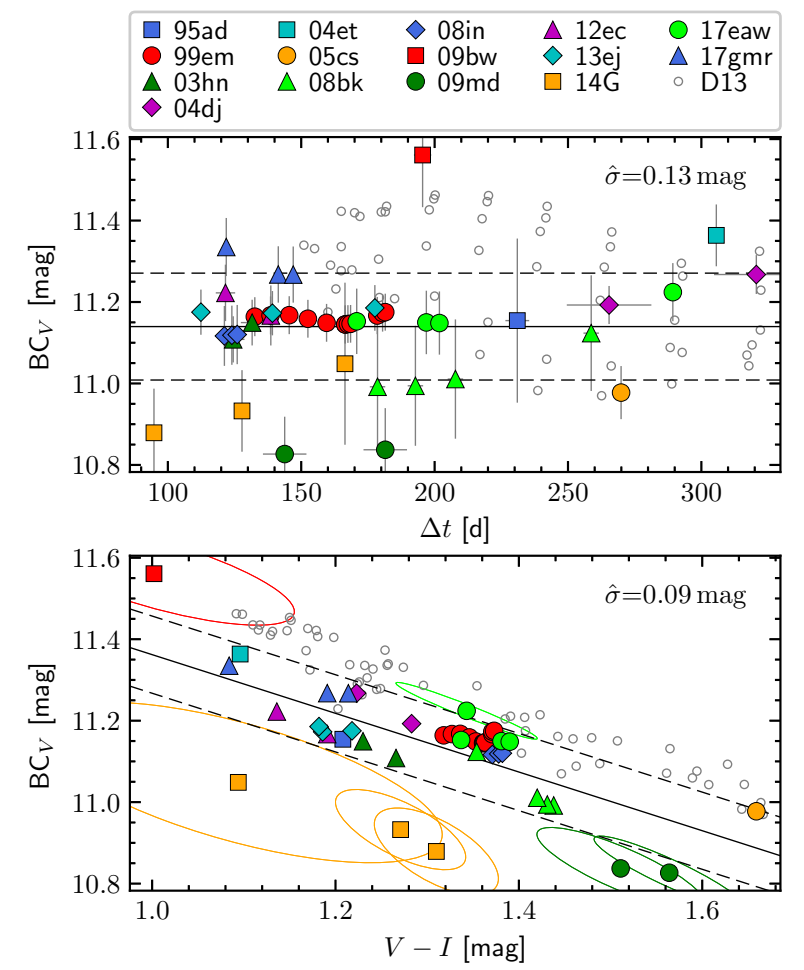

Figure 8. $\mathrm{BC}_{V}$ values as a function of time since explosion (top panel) and $V-I$ (bottom panel). Symbols, lines, error bars, and ellipses have the same meaning than in Fig. 7.

the confidence region of each observation is an elongated ellipse. We see that the confidence regions are nearly oriented in the direction of the $\mathrm{qBC}_{V}$ versus $V-I$ correlation. Therefore, the errors in $E_{B-V}^{\mathrm{G}}, E_{B-V}^{\mathrm{h}}$, and $V$-band photometry are not the main sources of the observed dispersion.

Fig. 8 shows $\mathrm{BC}_{V}$ against the time since explosion (top panel) and $V-I$ (bottom panel). As we can see in the figure, the behaviour of $\mathrm{BC}_{V}$ is the same as that of $\mathrm{qBC}_{V}$.

\subsection{BC calibration}

\subsection{1 $\mathrm{BC}_{V}$ versus $V-I$}

To calibrate the dependence of $\mathrm{BC}_{V}$ on $V-I$ displayed in the bottom panel of Fig. 8, we use the expression

$\mathrm{BC}_{x}=\mathrm{ZP}_{x}^{\mathrm{BC}}+\Psi_{x}(X)$.

Here, $\mathrm{ZP}_{x}^{\mathrm{BC}}$ is the zero-point for the $x$-band BC calibration, and $\Psi_{x}$ is a polynomial function (without the zero-order term) representing the dependence of $\mathrm{BC}_{x}$ on the independent variable $X$ (in our case, $X=V-I$ ). To compute the polynomial parameters, we minimize

$s^{2}=\sum_{\mathrm{SN}} \sum_{i}\left[\mathrm{BC}_{V, i}^{\mathrm{SN}}+\delta^{\mathrm{SN}}-\Psi_{V}\left((V-I)_{i}^{\mathrm{SN}}\right)\right]^{2}$,

where $\delta^{\mathrm{SN}}$ is an additive term to normalize the $\mathrm{BC}_{V}$ values of each SN to the same scale, and the polynomial order is determined with the model selection described in Appendix C.

The top panel of Fig. 9 shows the result of the aforementioned process, where we exclude SNe 1995ad, 2004et, $2005 \mathrm{cs}$, and $2009 \mathrm{bw}$ because they have only one $\mathrm{BC}_{V}$ esti- 

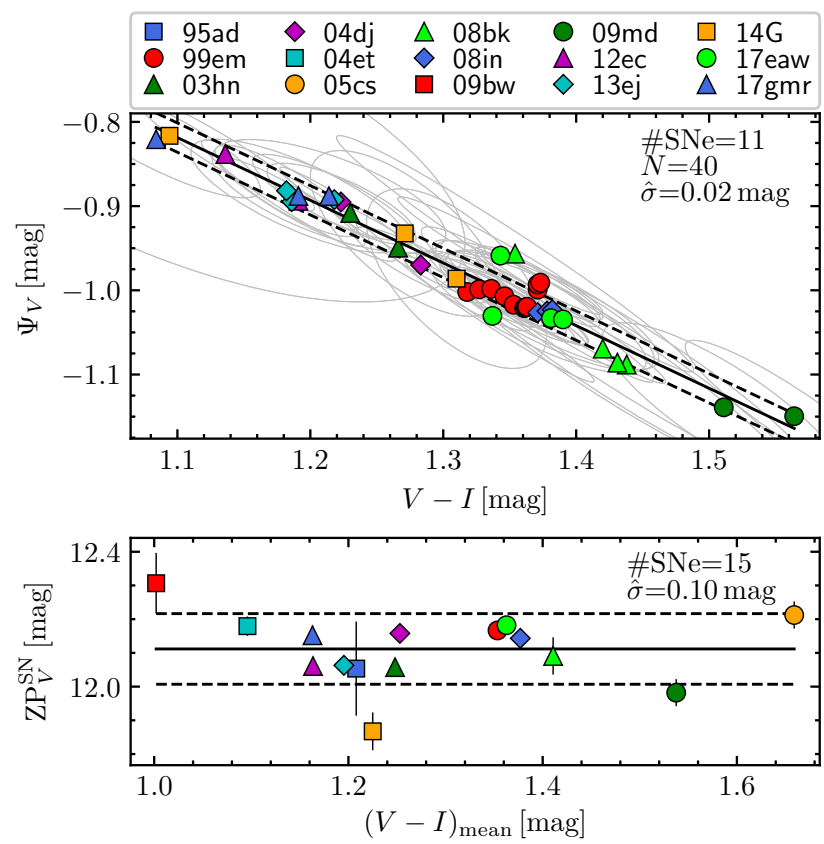

Figure 9. Top panel: $\Psi_{V}$ against $V-I$, where the solid line is a linear fit and ellipses are the $1 \sigma$ confidence regions. Bottom panel: $\mathrm{ZP}_{V}^{\mathrm{SN}}$ as a function of the mean $V-I$ colour, where the solid line indicates the mean value. Dashed lines are the $\pm 1 \hat{\sigma}$ limits.

mate. From this analysis we obtain that data are well represented by a straight line with slope $\left(\beta_{V}\right)$ of $-0.745 \pm 0.002$.

To compute the $\mathrm{ZP}_{V}^{\mathrm{BC}}$ value for each $\mathrm{SN}\left(\mathrm{ZP}_{V}^{\mathrm{SN}}\right)$, we arrange equations (24), (15), and (2), obtaining

$\mathrm{ZP}_{V}^{\mathrm{SN}}=\left\langle m_{\mathrm{bol}, i}-\left(1+\beta_{V}\right) V_{i}+\beta_{V} I_{i}\right\rangle+\left(E_{B-V}^{\mathrm{G}}+E_{B-V}^{\mathrm{h}}\right) \xi$,

where $\xi=\left(1+\beta_{V}\right) R_{\bar{\lambda}_{V}}-\beta_{V} R_{\bar{\lambda}_{I}}=2.01$ (using the $R_{\bar{\lambda}_{x}}$ values provided in Table A1). The angle brackets in equation (26) denote a weighted mean with weights

$w_{i}=\left[\sigma_{m_{\mathrm{bol}, i}}^{2}+\left(1+\beta_{V}\right)^{2} \sigma_{V_{i}}^{2}+\beta_{V}^{2} \sigma_{I_{i}}^{2}\right]^{-1}$,

where $\sigma_{m_{\mathrm{bol}, i}}$ only includes the error on photometry. The random error for each $\mathrm{ZP}_{V}^{\mathrm{SN}}$ value is given by

$\sigma_{\mathrm{ZP} S}=\left[\frac{1}{\sum_{i} w_{i}}+\left(\sigma_{E_{B-V}^{\mathrm{G}}}^{2}+\sigma_{E_{B-V}^{\mathrm{h}}}^{2}\right) \zeta^{2}\right]^{1 / 2}$,

where $\zeta=\xi-R_{\mathrm{p}}$, being $R_{\mathrm{p}}$ the pSED total-to-selective extinction ratio (see Appendix B). For our BC calibration set $R_{\mathrm{p}}=1.68$, so the $E_{B-V}^{\mathrm{G}}$ and $E_{B-V}^{\mathrm{h}}$ errors are scaled by 0.33 . For example, an $E_{B-V}^{\mathrm{h}}$ uncertainty of $0.08 \mathrm{mag}$ (the typical value for our sample) induces an error on $\mathrm{ZP}_{V}^{\mathrm{SN}}$ of $0.03 \mathrm{mag}$.

The bottom panel of Fig. 9 shows the $\mathrm{ZP}_{V}^{\mathrm{SN}}$ values for the $\mathrm{SNe}$ in the $\mathrm{BC}$ calibration set. To verify whether a residual correlation between $\mathrm{ZP}_{V}^{\mathrm{SN}}$ and $V-I$ exists, in the figure we plot the $\mathrm{ZP}_{V}^{\mathrm{SN}}$ values against mean $V-I$ colours. Using the model selection given in Appendix C, we find that data are consistent with $\mathrm{ZP}_{V}^{\mathrm{SN}}$ being constant, meaning that all the dependence of $\mathrm{ZP}_{V}^{\mathrm{SN}}$ on $V-I$ was captured by $\Psi_{V}$. We compute a mean and $\hat{\sigma}$ value of 12.11 and $0.10 \mathrm{mag}$, respectively. The typical $\mathrm{ZP}_{V}^{\mathrm{SN}}$ error is about $0.03 \mathrm{mag}$, so the observed $\hat{\sigma}$ value is mainly due to intrinsic differences between SNe. Therefore we adopt $\mathrm{ZP}_{V}^{\mathrm{BC}}=12.11 \pm 0.10 \mathrm{mag}$.
Table 2. BC calibrations.

\begin{tabular}{cccc}
\hline$x$ & $X$ & $\beta_{x}$ & $\mathrm{ZP}_{x}^{\mathrm{BC}}(\mathrm{mag})$ \\
\hline$V$ & $V-I$ & $-0.745 \pm 0.002$ & $12.11 \pm 0.10$ \\
$r$ & $r-I$ & $-0.837 \pm 0.004$ & $12.35 \pm 0.10$ \\
$R$ & $R-I$ & $-0.755 \pm 0.007$ & $12.35 \pm 0.10$ \\
$i$ & $i-I$ & $-0.963 \pm 0.005$ & $12.42 \pm 0.10$ \\
$I$ & $\Delta t /(100 \mathrm{~d})$ & $0.036 \pm 0.002$ & $12.37 \pm 0.10$ \\
$V$ & - & - & $11.15 \pm 0.18$ \\
$r$ & - & - & $11.89 \pm 0.16$ \\
$R$ & - & - & $12.07 \pm 0.14$ \\
$i$ & - & - & $11.91 \pm 0.12$ \\
$I$ & - & & $12.44 \pm 0.11$ \\
\hline Notes. & $\mathrm{BC}_{x}=\mathrm{ZP}_{x}^{\mathrm{BC}}+\beta_{x} X$, valid for $\Delta t$ between 95 and $320 \mathrm{~d}$. \\
ZP & BC & errors do not include the uncertainty due to the $\alpha$ error.
\end{tabular}

\subsubsection{BCs for other combinations}

In addition to $\mathrm{BC}_{V}$ as a function of $V-I$, we also calibrate the dependence of $\mathrm{BC}_{x}$ for the $\operatorname{VrRiI}$ bands as a function of different independent variables. In the $\mathrm{BC}$ calibration set there are only six SNe having ri photometry in the radioactive tail (namely SNe 2008in, 2012ec, 2013ej, 2014G, 2017eaw, and 2017gmr). For the remaining nine SNe we convert the $R I$ magnitudes to $r i$ ones (see Appendix $\mathrm{H}$ ).

To calibrate the dependence of $\mathrm{BC}_{x}$ on a given $X$ variable, we perform the same analysis as in Section 4.2.1. As independent variables, we consider the ten colour indices that can be defined with the VrRiI bands along with $\Delta t$. We find that the $\mathrm{BC}$ calibrations providing the lowest $\hat{\sigma}$ values are $\mathrm{BC}_{V}$ as a function of $V-I, \mathrm{BC}_{r}$ as a function of $r-I, \mathrm{BC}_{R}$ as a function of $R-I, \mathrm{BC}_{i}$ as a function of $i-I$, and $\mathrm{BC}_{I}$ as a function of $\Delta t$. In all cases the dependence of $\mathrm{BC}_{x}$ on the $X$ variable is linear. The slopes of the linear relations $\left(\beta_{x}\right)$ are reported in Column 3 of Table 2 .

The left-hand side of Fig. 10 shows the $\mathrm{ZP}_{x}^{\mathrm{SN}}$ values for the aforementioned calibrations. For each one we find that the $\mathrm{ZP}_{x}^{\mathrm{SN}}$ estimates are consistent with a constant value (using the model selection given in Appendix C). As for $\mathrm{BC}_{V}$ as a function of $V-I$, for $r R i I$ bands we adopt the mean and $\hat{\sigma}$ value as $\mathrm{ZP}_{x}^{\mathrm{BC}}$ and its error, respectively. Those values are listed in Column 4 of Table 2. Given the domain of the data (top panel of Fig. 7), our BC calibrations are valid for $\Delta t$ between 95 and $320 \mathrm{~d}$. Since the BC calibration set includes sub-luminous (SNe 2005cs, 2008bk, and 2009md) and moderately-luminous (e.g. SNe 2004et, 2009bw, and 2017gmr) SNe II, we assume that our $\mathrm{BC}_{x}$ calibrations are valid for all normal SNe II.

We also compute $\mathrm{BC}_{x}$ calibrations assuming they are constant. The $\mathrm{ZP}_{x}^{\mathrm{BC}}$ values are given in Column 4 of Table 2, while the $\mathrm{ZP}_{x}^{\mathrm{SN}}$ estimates are shown in the right-hand side of Fig. 10. Since $\mathrm{BC}_{x}$ is not actually constant but depends on a specific $X$ variable, there is a dependence of $\mathrm{ZP}_{x}^{\mathrm{SN}}$ on the mean $X$ values. The latter, as we can see in right-hand side of Fig. 10, is more evident for $\operatorname{VrR}$ bands.

The BCs with the best precision are those including the $I$-band in the calibration $(\hat{\sigma}=0.10-0.11 \mathrm{mag})$, followed by $\mathrm{BC}_{i}$ as a constant $(\hat{\sigma}=0.12 \mathrm{mag})$. The latter means that, among the VrRiI bands, the $I$ - and $i$-band magnitudes are more correlated with the bolometric one. In order to compute luminosities through the $\mathrm{BC}$ technique (equation 17), the $I$-band photometry along with $\mathrm{BC}_{I}$ as a function of $\Delta t$ must be preferred. If the $I$-band photometry is not avail- 

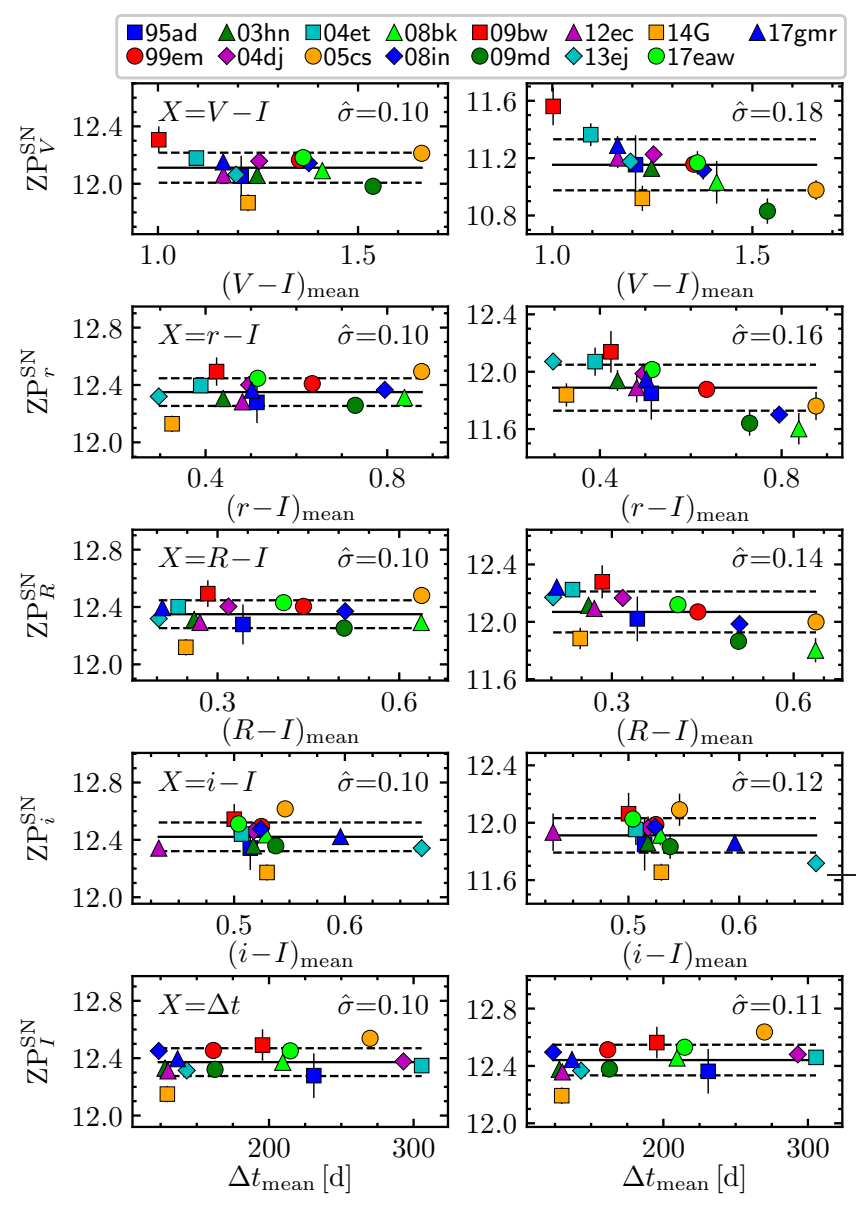

Figure 10. $\mathrm{ZP}_{x}^{\mathrm{SN}}$ versus the mean $X$ value. Left-hand panels: using the calibration with the lowest $\hat{\sigma}$ value. Right-hand panels: assuming a constant BC. Solid and dashed lines indicate mean values and $\pm 1 \hat{\sigma}$ limits around the mean, respectively.

able, then the $i_{-}, R-, r_{-}$, or $V$-band photometry along with the constant $\mathrm{BC}_{x}$ can be used to estimate luminosities. In the latter case, however, we caution that the derived luminosities of sub-luminous and moderately-luminous SNe II will be systematically under and overestimated, respectively, specially for the $\operatorname{VrR}$ bands.

\section{$4.3 \quad{ }^{56} \mathrm{Ni}$ mass distribution}

\subsection{1 $\log M_{56 \mathrm{Ni}}$ estimates}

Armed with BCs for normal SNe II in the radioactive tail, we compute $\log M_{56} \mathrm{Ni}$ using the recipe given in Appendix D.

For $25 \mathrm{SNe}$ in our sample we find it is necessary to correct for the deposition function. As example, the left-hand side of Fig. 11 shows the $\log M_{56} \mathrm{Ni}$ estimates of SN 2014G as a function of the time since explosion, assuming the complete $\gamma$-ray trapping scenario (i.e. $D_{i}=0$ in equation 18). As visible in the figure, the $\log M_{56 \mathrm{Ni}}$ estimates are not concentrated around a constant but they decrease with time. This trend emerges because the ejecta becomes less able to thermalize $\gamma$-rays with time. In this case of $\gamma$-ray leakage, the inferred $\log M_{56 \mathrm{Ni}}$ corresponds to a lower limit. The right-hand side of the figure shows the $\log M_{56 \mathrm{Ni}}$ estimates corrected for $f_{\text {dep }}$. We can see that, using $T_{0}=127 \mathrm{~d}$, the
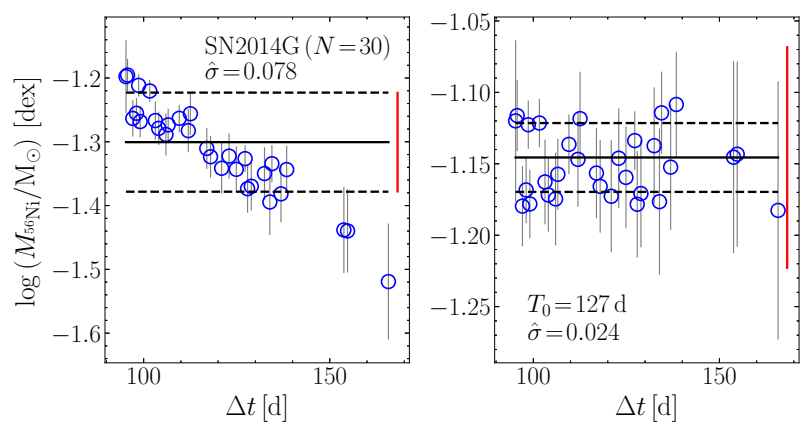

Figure 11. $\log M_{56} \mathrm{Ni}$ estimates of SN $2014 \mathrm{G}$ versus the time since explosion, assuming the complete $\gamma$-ray trapping scenario (lefthand panel), and correcting for $f_{\text {dep }}$ (right-hand panel). Solid horizontal lines correspond to the $\log M_{56} \mathrm{Ni}$ values that maximize the likelihood, and dashed lines are the $\pm 1 \hat{\sigma}$ limits around them. Error bars are $1 \sigma$ errors due to uncertainties on photometry, while red vertical bars depict the $1 \sigma$ errors due to the uncertainty on $\mu$, colour excesses, $t_{0}$, and $\mathrm{BC}$.

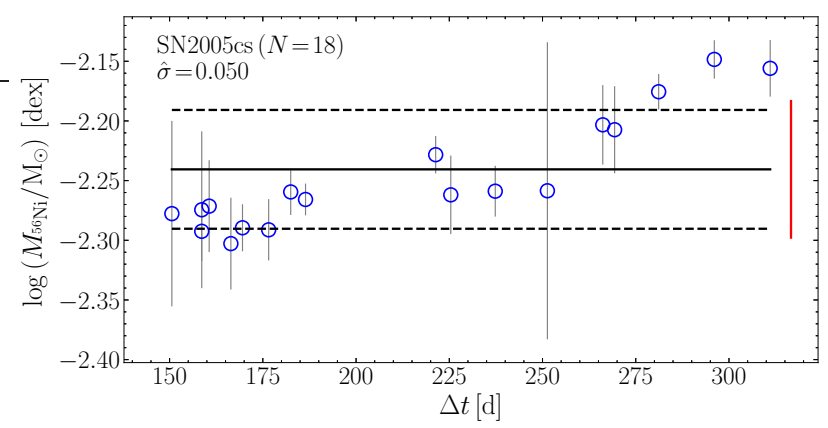

Figure 12. $\log M_{56} \mathrm{Ni}$ estimates of SN 2005cs versus the time since explosion, assuming the complete $\gamma$-ray trapping scenario. Error bars and lines have the same meaning than in Fig. 11.

systematic with time disappears and the $\log M_{56_{\mathrm{Ni}}}$ estimates are consistent with a constant value.

In our SN set, SNe 2004dj, 2005cs, 2006my, 2013am, and $2013 \mathrm{bu}$ have $\log M_{56 \mathrm{Ni}}$ estimates that increase with time, which is shown in Fig. 12 for the case of SN 2005cs. This trend indicates that the observed luminosity increases with time relative to that expected from the radioactive decay. The latter suggests that (1) the SN ejecta during the early radioactive tail is not optically thin enough, so the energy deposited in the ejecta is not immediately emitted (i.e. $L<Q_{\text {dep }}$ ); and/or (2) there is an additional source of energy (i.e. $L>Q_{\mathrm{dep}}$ ), whose relative contribution to the observed flux increases with time. For the latter scenarios, the higher and lower $\log M_{56} \mathrm{Ni}$ estimates are closer to the real value, respectively. In this work, in order to be conservative about the origin of the observed tendency, for the aforementioned five SNe we adopt the $\log M_{56 \mathrm{Ni}}$ value obtained with $D_{i}=0$.

In the case of SNe 1988A, 2003iq, 2005dx, PTF10gva, 2010aj, LS13dpa, 2015cz, and 2016ija we obtain $D_{i}=0$ because the characteristics of their photometry (number of data, photometry errors, and time baseline) are not good enough to detect departures from a constant $\log M_{56} \mathrm{Ni}$ value. In our sample 30 out of $102 \mathrm{SNe}$ are consistent with $D_{i} \neq 0$. Therefore, we expect only two out of the aforementioned SNe to have $D_{i} \neq 0$, which should not impact our results. 
Table 3. Error budget for the $\log M_{56 \mathrm{Ni}}$ estimates.

\begin{tabular}{llccc}
\hline $\begin{array}{l}\text { Error } \\
\text { type }\end{array}$ & $\begin{array}{l}\text { Error } \\
\text { source }\end{array}$ & $\begin{array}{c}\text { Typical } \\
\text { error }\end{array}$ & $\begin{array}{c}\text { Error in } \\
\log M_{56} \mathrm{Ni} \\
(\mathrm{dex})\end{array}$ & $\begin{array}{c}\% \text { of total } \\
\text { error }\end{array}$ \\
\hline Random & $\mu$ & $0.18 \mathrm{mag}$ & 0.072 & 50.4 \\
& $E_{B-V}^{\mathrm{h}}$ & $0.08 \mathrm{mag}$ & 0.054 & 28.0 \\
& $t_{0}$ & $3.8 \mathrm{~d}$ & 0.013 & 1.6 \\
& $m_{I}$ & $0.05 \mathrm{mag}$ & $0.012^{*}$ & 1.3 \\
& $E_{B}^{\mathrm{G}}-V$ & $0.01 \mathrm{mag}$ & 0.007 & 0.4 \\
Systematic & $\mathrm{All}$ & & 0.092 & 81.7 \\
& $\mathrm{ZP}$ & $0.10 \mathrm{mag}$ & 0.040 & 15.5 \\
& $\alpha$ & $3.9 \%$ & 0.017 & 2.8 \\
& $\mathrm{All}$ & & 0.043 & 18.3 \\
Total & & & 0.102 & 100.0 \\
\hline * Considerin & & &
\end{tabular}

${ }^{*}$ Considering three photometric points.

Table L5 lists the derived $\log M_{56 \mathrm{Ni}}$ and $M_{56 \mathrm{Ni}}$ values (Columns 6 and 7, respectively), along with the bands and numbers of photometric points used to compute $\log M_{56 \mathrm{Ni}}$ (Columns 2 and 4, respectively). The $T_{0}$ values of the $24 \mathrm{SNe}$ corrected for the deposition function are listed in Column 5.

Table 3 shows the error budget for the $\log M_{56} \mathrm{Ni}$ estimates computed with the $I$-band photometry (the preferred one), adopting the typical errors in our SN sample. The uncertainty on $\mu$ dominates the error budget, accounting for about 50 per cent of the total error. The $\mathrm{ZP}_{I}^{\mathrm{BC}}$ error, on the other hand, is the main source of systematic uncertainty. Errors in photometry, $t_{0}$, and $E_{B-V}^{\mathrm{G}}$ induce only $\sim 3$ per cent of the total $\log M_{56}{ }_{\mathrm{Ni}}$ error. The typical error of the measured $\log M_{56_{\mathrm{Ni}}}$ is of $0.102 \mathrm{dex}\left(M_{56} \mathrm{Ni}\right.$ error of 24 per cent).

Similar to the $I$-band, the $\log M_{56 \mathrm{Ni}}$ error budgets for the $V r R i$ bands are dominated by uncertainties on $\mu$ and $\mathrm{ZP}_{x}^{\mathrm{BC}}$. Errors in photometry, $t_{0}$, and $E_{B-V}^{\mathrm{G}}$ induce about 2-3 per cent of the total $\log M_{56 \mathrm{Ni}}$ error, while the typical $\log M_{56 \mathrm{Ni}}$ errors are of $0.143,0.128,0.121$, and $0.109 \mathrm{dex}$ for the $\operatorname{VrR} i$ bands, respectively.

\subsubsection{Outliers}

Fig. 13 shows $\log M_{56} \mathrm{Ni}$ versus the absolute $V$-band magnitude at $50 \mathrm{~d}$ since the explosion $\left(M_{V}^{50 \mathrm{~d}}\right.$, listed in Column 8 of Table L5). As reported by Hamuy (2003) and other authors (e.g. Spiro et al. 2014, Pejcha \& Prieto 2015a,b, Valenti et al. 2016, Müller et al. 2017, Singh et al. 2019), we see a correlation between both quantities. As noted by Pejcha \& Prieto (2015a), SN 2007od is an outlier in the $\log M_{56 \mathrm{Ni}}$ versus $M_{V}^{50 \mathrm{~d}}$ distribution. The latter is a consequence of the increase in extinction due to the newly formed dust during the radioactive tail (Andrews et al. 2010; Inserra et al. 2011)

Using the model selection procedure (Appendix C), we find that the correlation between $\log M_{56 \mathrm{Ni}}$ and $M_{V}^{50 \mathrm{~d}}$ can be represented by the straight line

$\log M_{56 \mathrm{Ni}}=-7.708( \pm 0.445)-0.3755( \pm 0.0271) M_{V}^{50 \mathrm{~d}}$,

where the parameter errors (in parentheses) are obtained performing $10^{4}$ bootstrap resamplings.

To identify outliers other than SN 2007od in the $\log M_{56}{ }_{\mathrm{Ni}}$ versus $M_{V}^{50 \mathrm{~d}}$ distribution, we use the Chauvenet's criterion. In Fig. 13 we see that SN 2010aj is located below the Chauvenet lower rejection limit but consistent with it within $2 \sigma$, which means that we cannot confirm that SN as

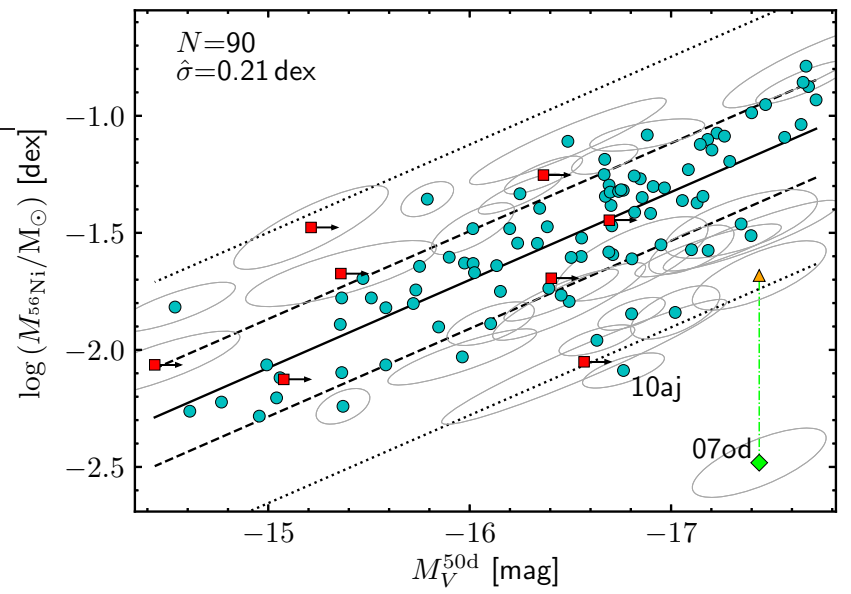

Figure 13. $\log M_{56} \mathrm{Ni}$ against $M_{V}^{50 \mathrm{~d}}$. The solid line is a straight-line fit to the data (cyan circles, $90 \mathrm{SNe}$ ), dashed lines indicate the $\pm 1 \hat{\sigma}$ limits around the fit, while dotted lines are the Chauvenet rejection limits. SNe with upper limits on $M_{V}^{50 \mathrm{~d}}$ are indicated as red squares. The green diamond corresponds to SNe 2007od, while the orange triangle is $\mathrm{SN} 2007 \mathrm{od}$ with a $\log M_{56} \mathrm{Ni}$ correction of 0.8 dex. Ellipses indicate $1 \sigma$ confidence regions, which for clarity are drawn only for $\mathrm{SNe}$ outside the $\pm 1 \hat{\sigma}$ limits.

an outlier. SN 2010aj was presented in Inserra et al. (2013), which suggested that it may be affected by newly formed dust. In that work, however, the lack of further evidence did not allow confirmation of the above scenario.

In the case of SNe 2007od we consider its $\log M_{56 \mathrm{Ni}}$ values as lower limits. Indeed, based on the $M_{56} \mathrm{Ni}$ values reported by Inserra et al. (2011), the inclusion of the IR light excess to the luminosity of SN 2007 od increases its $\log M_{56} \mathrm{Ni}$ in $\sim 0.8$ dex. Applying this correction, SN 2007od moves in Fig. 13 from -6.3 to $-2.5 \hat{\sigma}$ below the fit, becoming consistent with the $\log M_{56} \mathrm{Ni}$ versus $M_{V}^{50 d}$ distribution.

\subsubsection{Sample completeness}

The $\mathrm{SNe}$ in our set were selected from the literature by having at least three photometric points in the radioactive tail, so our sample is potentially affected by the selection bias. In order to correct for the latter bias and construct an SN sample as complete as possible, we use as reference the volume-limited SN sample of Shivvers et al. (2017). Most of the SNe II in that sample have completeness $\gtrsim 95$ per cent at the cut-off distance of $38 \mathrm{Mpc}(\mu=32.9 \mathrm{mag})$, while sub-luminous and highly reddened SNe have completeness $\gtrsim 70$ per cent (see Fig. 4 of $\mathrm{Li}$ et al. 2011). Therefore, we assume that the set of normal SNe II at $\mu<32.9$ in the Shivvers et al. (2017) sample is roughly complete.

From the Shivvers et al. (2017) sample we select the 28 normal SNe II with $\mu<32.9$ mag (hereafter the RC set), where we use $\mu$ values computed with the procedure described in Section 3.6.1 (reported in Column 8 of Table L2). We recalibrate their absolute $R$ magnitudes $\left(M_{R}\right)$ at maximum $\left(M_{R}^{\max }\right.$, listed in Li et al. 2011) using our $\mu$ values, and correcting for $E_{B-V}^{\mathrm{h}}$ (estimated with the procedure described in Section 3.6.2, and reported in Column 6 of Table L3). We also replace the Schlegel et al. (1998) $E_{B-V}^{\mathrm{G}}$ values used in $\mathrm{Li}$ et al. (2011) by the new ones provided by 

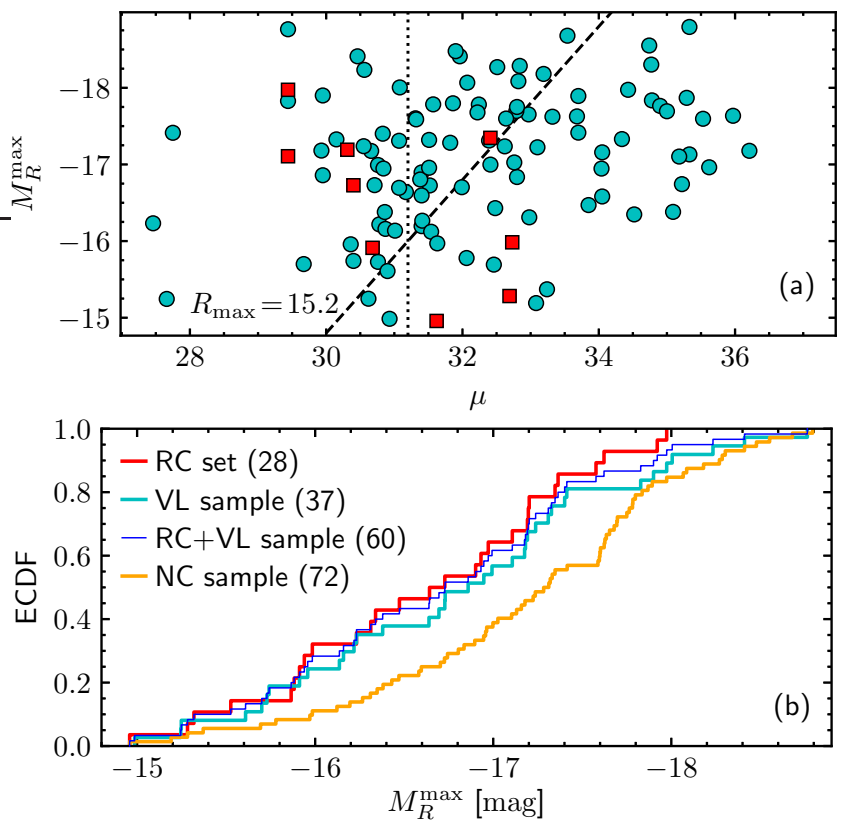

Figure 14. Panel (a): $M_{R}^{\max }$ against $\mu$ for the $\mathrm{SNe}$ in our sample, where red squares are the $\mathrm{SNe}$ in common with the RC set. The dashed line corresponds to $R_{\max }=15.2 \mathrm{mag}$, while the dotted line $(\mu=31.2)$ is the limit for our VL sample. Panel (b): ECDF for the $M_{R}^{\max }$ values in the RC (red line), VL (cyan line), and $\mathrm{RC}+\mathrm{VL}$ (thin blue line) samples, and for the $M_{R}^{\max }$ values of the $\mathrm{SNe}$ in our sample with $\mu>31.2$ (orange line).

Schlafly \& Finkbeiner (2011). Table L6 lists the $M_{R}^{\max }$ estimates for the RC sample.

Among the $109 \mathrm{SNe}$ in our sample, nine have $M_{R}^{\max }$ estimates provided in the $\mathrm{RC}$ set, so we adopt those values for consistency. Out of the remaining $100 \mathrm{SNe}$

1. Forty-seven SNe have $R$ - or $r$-band light curves during the maximum light, where $r$ magnitudes are converted into $R$ ones using $r-R=0.12$ (see Appendix $\mathrm{H}$ ). We measure $M_{R}^{\max }$ performing an ALR fit to the maximum photometry or adopting the brightest $M_{R}$ value $\left(M_{R}^{\mathrm{br}}\right)$ as $M_{R}^{\max }$. The average of the rise time $\left(t_{R}^{\text {rise }}\right)$ for the latter SNe is of $15 \pm 6 \mathrm{~d}$ ( $1 \hat{\sigma}$ error), while the mean $V-R$ and $R-I$ colours at time $t_{R}^{\text {rise }}$ are of $0.15 \pm 0.08$ and $0.04 \pm 0.07 \mathrm{mag}$, respectively.

2 . Thirty $\mathrm{SNe}$ have $R / r$ light curves where the maximum light cannot be determined; six SNe have $V I$ photometry, which we convert into $R$ magnitudes using $V-R=0.05+0.59(V-I)$ (see Appendix $\mathrm{H})$; and eight $\mathrm{SNe}$ (one $\mathrm{SN}$ ) only have $V$-band ( $I$-band) photometry, which we convert into $R$ magnitudes using $V-R=0.15(R-I=0.04)$. For each of these $45 \mathrm{SNe}$ we fit a straight line to the light curve, compute $M_{R}$ at $t_{R}^{\text {rise }}\left(M_{R}\left(t_{R}^{\text {rise }}\right)\right)$, and adopt the minimum between $M_{R}^{\mathrm{br}}$ and $M_{R}\left(t_{R}^{\mathrm{rise}}\right)$ as $M_{R}^{\max }$.

3. Eight SNe have photometry starting at $\Delta t>80 \mathrm{~d}$. Two of them (SNe 1997D and 2004eg) are sub-luminous SNe II, which tend to have flat light curves during the photospheric phase (e.g. Spiro et al. 2014), so we adopt $M_{R}^{\mathrm{br}}$ as $M_{R}^{\max }$. For the remaining six $\mathrm{SNe}$ we estimate $M_{R}^{\max }$ using their $\log M_{56}{ }_{\mathrm{Ni}}$ values and $M_{R}^{\max }=-20.01-1.86 \log M_{56 \mathrm{Ni}}$, obtained from the $47 \mathrm{SNe}$ with well-defined maximum light.

The $M_{R}^{\max }$ values for the SNe in our sample are reported in Table L6 and plotted in Fig. 14(a) against $\mu$. The mean apparent $R$-band magnitude at maximum ( $R_{\max }$, corrected for reddening) is of $15.2 \mathrm{mag}$, which is indicated as a dashed line. As we move to greater $R_{\max }$ values (right-hand side of the dashed line) we see a decrement in the number of SNe, which is due to (1) bright $\mathrm{SNe}$ (in apparent magnitude) are more likely to be selected for photometric monitoring in the radioactive tail than faint ones; and (2) faint SNe have in general less photometric points in the radioactive tail than bright ones, so they are more likely not to meet our selection criterion of having at least three photometric points. In order to minimize the effect of the selection bias, we construct a volume-limited (VL) sample with the $37 \mathrm{SNe}$ at $\mu \leq 31.2$ such that the selection bias could be relevant only in the small region between $R_{\max }>15.2$ and $\mu \leq 31.2$.

Fig. 14(b) shows the empirical cumulative distribution function (ECDF) for the $M_{R}^{\max }$ values in the VL (cyan line) and the RC (red line) samples. To test whether both $M_{R}^{\max }$ samples are drawn from a common unspecified distribution (the null hypothesis), we use the two-sample AndersonDarling (AD) test (e.g. Scholz \& Stephens 1987). We obtain a standardized test statistic $\left(T_{\mathrm{AD}}\right)$ of -0.65 with a $p$-value of 0.74 , meaning that the null hypothesis cannot be rejected at a significance level $>74$ per cent. Since the $M_{R}^{\max }$ values of the RC and the VL samples are likely drawn from the same $M_{R}^{\max }$ distribution, we can assume that the completeness of both samples is quite similar, so we combine them into a single data set $(\mathrm{RC}+\mathrm{VL})$. The $M_{R}^{\max } \mathrm{ECDF}$ of the $\mathrm{RC}+\mathrm{VL}$ sample (blue thin line) has a minimum, maximum, mean and $\hat{\sigma}$ of $-18.8,-15.0,-16.7$, and $0.9 \mathrm{mag}$, respectively.

Fig. 14(b) also shows the ECDF for the $M_{R}^{\max }$ values of the $72 \mathrm{SNe}$ at $\mu>31.2$ (orange line), which we refer as the non-complete $(\mathrm{NC})$ sample. Using the two-sample AD test to test the null hypothesis for the $M_{R}^{\max }$ values of the $\mathrm{RC}+\mathrm{VL}$ and the NC samples, we obtain a $T_{\mathrm{AD}}$ of 5.66 and a $p$-value of 0.002 . Thus, the null hypothesis can be rejected at a significance level of 0.2 per cent, which is expected since the NC sample is affected by the selection bias.

In order to roughly quantify the number and magnitudes of the SNe missing from the NC sample, and therefore from our full sample, we proceed as follows. First, we divide the $M_{R}^{\max }$ distribution into four bins of width 1 mag (Column 1 of Table 4), and register the number of SNe within each bin for the $\mathrm{NC}$ and the RC+VL samples (Columns 2 and 3, respectively). Then, since bright $\mathrm{SNe}$ are less affected by the selection bias, we scale the number of $\mathrm{SNe}$ in the $\mathrm{RC}+\mathrm{VL}$ sample by a factor of $15 / 8$ (Column 4) in order to match the number of SNe with $M_{R}^{\max }<-17.8$ to that of the NC sample. In other words, the numbers in Column 4 are the SNe we would expect for a roughly complete sample with $15 \mathrm{SNe}$ at $M_{R}^{\max }<-17.8$. The number of expected $\mathrm{SNe}$ minus the observed ones (i.e. the NC sample) is listed in Column 5. Thus, to correct our full SN sample for selection bias, we have to include 3,23 , and 15 SNe of magnitude $-17.8 \leq M_{R}^{\max }<-16.8,-16.8 \leq M_{R}^{\max }<-15.8$, and $M_{R}^{\max } \geq-15.8$, respectively. The latter $\mathrm{SNe}$ can be randomly selected from the VL or the RC+VL sample within the corresponding $M_{R}^{\max }$ bins.

\subsubsection{Mean ${ }^{56}$ Ni mass}

Fig. 15 shows the ECDFs for the $M_{56} \mathrm{Ni}$ values in the VL (cyan line) and the full (blue line) samples. The ECDF 
Table 4. Histogram for $M_{R}^{\max }$.

\begin{tabular}{lcccc}
\hline$M_{R}^{\max }$ range & $\mathrm{NC}$ & $\mathrm{RC}+\mathrm{VL}$ & Expected & Missing \\
\hline$-17.8,-18.8$ & 15 & 8 & 15 & 0 \\
$-16.8,-17.8$ & 36 & 21 & 39 & 3 \\
$-15.8,-16.8$ & 15 & 20 & 38 & 23 \\
$-14.8,-15.8$ & 6 & 11 & 21 & 15 \\
\hline Notes: Expected $=15 / 8(\mathrm{RC}+\mathrm{VL})$. Missing = Expected - NC.
\end{tabular}

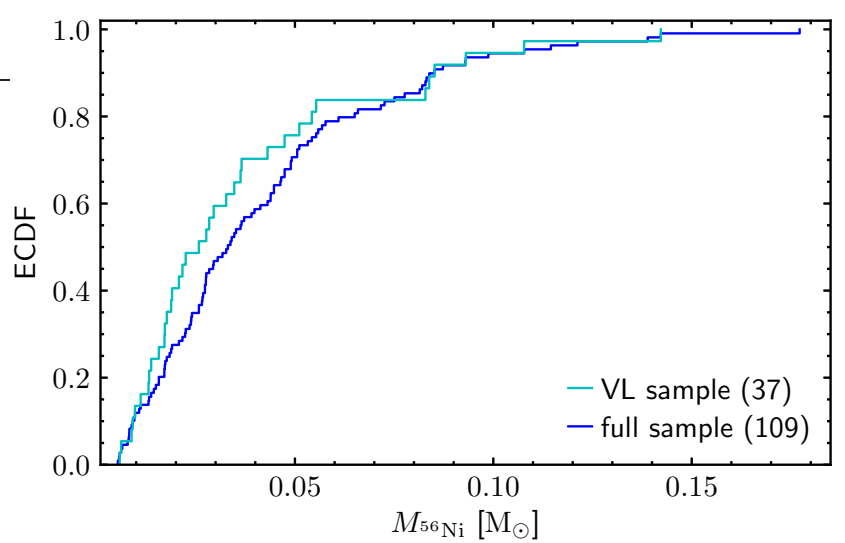

Figure 15. ECDF for the ${ }^{56} \mathrm{Ni}$ masses in the VL (cyan line) and the full sample (blue line).

of the VL sample has a $\left\langle M_{56} \mathrm{Ni}\right\rangle$ and $\hat{\sigma}$ of 0.037 and $0.032 \mathrm{M}_{\odot}$, respectively, while the random error (ran) on the mean $(\hat{\sigma} / \sqrt{N})$ is of $0.005 \mathrm{M}_{\odot}$. On the other hand, the $M_{56} \mathrm{Ni}$ distribution of the full sample has a minimum, maximum, mean, and $\hat{\sigma}$ of $0.005,0.177,0.042$, and $0.033 \mathrm{M}_{\odot}$, respectively, with $\hat{\sigma} / \sqrt{N}=0.003 \mathrm{M}_{\odot}$. The latter mean value corresponds to $\left\langle M_{56} \mathrm{Ni}\right\rangle$ uncorrected for selection bias $\left(\left\langle M_{56}{ }_{\mathrm{Ni}}\right\rangle^{\mathrm{unc}}\right)$ which, as expected, is greater than the $\left\langle M_{56} \mathrm{Ni}\right\rangle$ estimate for the VL sample. We note that for models based on the neutrino-heating mechanism (the generally accepted one for CC SNe, e.g. Burrows \& Vartanyan 2021) the upper limit for the synthesized $M_{56} \mathrm{Ni}$ is around 0.15 and $0.23 \mathrm{M}_{\odot}$ (e.g. Ugliano et al. 2012; Suwa et al. 2019), which is consistent with the maximum $M_{56} \mathrm{Ni}$ of our full SN sample.

The random error on $\left\langle M_{56 \mathrm{Ni}}\right\rangle^{\text {unc }}$ is made up of the sampling error along with the uncertainties induced by errors in $\mu$ and $E_{B-V}^{\mathrm{h}}$. To estimate the random error in $\left.\left\langle M_{56 \mathrm{Ni}}\right\rangle\right\rangle^{\text {unc }}$ induced by uncertainties in $\mu$, we perform $10^{5}$ simulations varying randomly $\mu$ according to its errors (assumed normal). For each realization, we rescale the $\log M_{56} \mathrm{Ni}$ values of the $\mathrm{SNe}$ in our full sample using the simulated $\mu$ values and calculate the mean ${ }^{56} \mathrm{Ni}$ mass. Using those $10^{5}$ simulated mean values we compute a $\hat{\sigma}$ around $\left\langle M_{56} \mathrm{Ni}^{\text {unc }}\right\rangle^{\text {of }}$ $0.0009 \mathrm{M}_{\odot}$, which we adopt as the error induced by uncertainties in $\mu$. We repeat the same process for $E_{B-V}^{\mathrm{h}}$, obtaining $0.0011 \mathrm{M}_{\odot}$. The random error on $\left\langle M_{56} \mathrm{Ni}\right\rangle^{\text {unc }}$ is $0.0028 \mathrm{M}_{\odot}$ greater in quadrature than the error induced by $\mu$ and $E_{B-V}^{\mathrm{h}}$. We adopt the latter value as the sampling error.

As mentioned in Section 4.3.3, to correct our full SN sample for selection bias we have to include $41 \mathrm{SNe}$. The mean ${ }^{56} \mathrm{Ni}$ mass of the selection-bias-corrected sample can be written as

$\left\langle M_{56_{\mathrm{Ni}}}\right\rangle=\left\langle M_{56 \mathrm{Ni}}\right\rangle^{\mathrm{unc}}-\mathrm{sbc}$.
Table 5. Error budget for the mean ${ }^{56} \mathrm{Ni}$ mass.

\begin{tabular}{llccc}
\hline Error type & $\begin{array}{l}\text { Error } \\
\text { source }\end{array}$ & $\begin{array}{c}\text { Typical } \\
\text { error }\end{array}$ & $\begin{array}{c}\text { Error in } \\
\left\langle M_{56} \mathrm{Ni}\right\rangle \\
\left(\mathrm{M}_{\odot}\right\rangle\end{array}$ & $\begin{array}{c}\text { \% of total } \\
\text { error }\end{array}$ \\
\hline Random & Sampling & $0.0028 \mathrm{M}_{\odot}$ & 0.0028 & 31.0 \\
& sbc & $0.0014 \mathrm{M}_{\odot}$ & 0.0014 & 7.7 \\
& $E_{B-V}^{\mathrm{h}}$ & $0.08 \mathrm{mag}$ & 0.0011 & 4.8 \\
& $\mu$ & $0.18 \mathrm{mag}$ & 0.0009 & 3.2 \\
Systematic & $\mathrm{All}$ & $0.10 \mathrm{mag}$ & 0.0034 & 45.6 \\
& $\mathrm{ZP}$ & $3.9 \%$ & 0.0014 & 7.7 \\
& $\alpha$ & & 0.00368 & 53.3 \\
Total & $\mathrm{All}$ & & 0.00504 & 100.0 \\
\hline
\end{tabular}

Here,

$\mathrm{sbc}=\frac{41}{109+41}\left(\left\langle M_{56_{\mathrm{Ni}}}\right\rangle^{\mathrm{unc}}-\left\langle M_{56_{\mathrm{Ni}}}\right\rangle_{41}^{\mathrm{unc}}\right)$

is the selection bias correction, where $\left\langle M_{56} \mathrm{Ni}\right\rangle_{41}^{\text {unc }}$ is the mean ${ }^{56} \mathrm{Ni}$ mass computed with the $41 \mathrm{SNe}$ that we have to add to our full SN sample. Performing $10^{5}$ simulations, where the missing SNe (Column 5 of Table 4) are randomly selected from the VL sample within the corresponding $M_{R}^{\max }$ bins, we obtain a sbc of $0.005 \pm 0.001 \mathrm{M}_{\odot}$. Therefore, our best estimate of $\left\langle M_{56} \mathrm{Ni}\right\rangle$ for normal $\mathrm{SNe}$ II is of $0.037 \pm 0.003(\mathrm{ran}) \mathrm{M}_{\odot}$, with a systematic error due to the uncertainty on $\mathrm{ZP}^{\mathrm{BC}}$ and $\alpha$ of $0.004 \mathrm{M}_{\odot}$. This result compares to the $\left\langle M_{56_{\mathrm{Ni}}}\right\rangle$ value of $0.037 \pm 0.005(\operatorname{ran}) \mathrm{M}_{\odot}$ obtained with the VL sample.

Table 5 summarizes the error budget for $\left\langle M_{56 \mathrm{Ni}}\right\rangle$. The $\mathrm{ZP}_{I}^{\mathrm{BC}}$ error, accounting for 46 per cent of the total uncertainty, dominates the error budget. The sampling error, which is the main source of random uncertainty, accounts for 31 per cent of the total error.

\subsection{Mean iron yield}

With our $\left\langle M_{56} \mathrm{Ni}\right\rangle$ measurement along with equation (21) and $\eta=1.07 \pm 0.04$ (see Section 3.5), we obtain a $\bar{y}_{\mathrm{Fe}}$ value of $0.040 \pm 0.005 \mathrm{M}_{\odot}$ for normal SNe II.

In addition, we evaluate $\bar{y}_{\mathrm{Fe}}$ for $\mathrm{CC} \mathrm{SNe}$ employing recent estimations of mean ${ }^{56} \mathrm{Ni}$ masses for other CC SN subtypes. The mean ${ }^{56} \mathrm{Ni}$ mass for $\mathrm{CC} \mathrm{SNe}$, using the $\mathrm{SN}$ rates provided in Shivvers et al. (2017), is given by

$f_{\mathrm{CC}}^{\mathrm{Ni}}=0.696 f_{\mathrm{II}}^{\mathrm{Ni}}+0.304 f_{\mathrm{SE}}^{\mathrm{Ni}}$.

Here,

$f_{\mathrm{II}}^{\mathrm{Ni}}=0.891 f_{\mathrm{II}-\text { normal }}^{\mathrm{Ni}}+0.067 f_{\mathrm{IIn}}^{\mathrm{Ni}}+0.042 f_{\text {long-rising }}^{\mathrm{Ni}}$

and

$f_{\mathrm{SE}}^{\mathrm{Ni}}=0.360 f_{\mathrm{IIb}}^{\mathrm{Ni}}+0.356 f_{\mathrm{Ib}}^{\mathrm{Ni}}+0.247 f_{\mathrm{Ic}}^{\mathrm{Ni}}+0.037 f_{\mathrm{Ic}-\mathrm{BL}}^{\mathrm{Ni}}$,

where $f^{\mathrm{Ni}}$ denotes the mean ${ }^{56} \mathrm{Ni}$ mass for the subscripted CC SN types and subtypes.

In equation $(33)$ we adopt $f_{\text {long-rising }}^{\mathrm{Ni}}=0.086 \mathrm{M}_{\odot}$ (Anderson 2019) and for SNe IIn we assume $f_{\mathrm{In}}^{\mathrm{Ni}}=f_{\mathrm{II}-\text { normal }}^{\mathrm{Ni}}$, thus obtaining $f_{\mathrm{II}}^{\mathrm{Ni}}=0.039 \mathrm{M}_{\odot}$.

For SNe IIb, Ib, Ic and Ic-BL we adopt the mean $M_{56 \mathrm{Ni}}$ values from the compilation of Anderson (2019): $0.124,0.199,0.198$, and $0.507 \mathrm{M}_{\odot}$, respectively. The ${ }^{56} \mathrm{Ni}$ masses of the SE SNe compiled by Anderson (2019) were 
mainly computed with the Arnett (1982) rule, which overestimates the ${ }^{56} \mathrm{Ni}$ mass of $\mathrm{SE} \mathrm{SNe}$ by $\sim 50$ per cent (Dessart et al. 2015, 2016). Including this correction to the mean $M_{56 \mathrm{Ni}}$ values of SE SNe, with equation (34) we get $f_{\mathrm{SE}}^{\mathrm{Ni}}=0.122 \mathrm{M}_{\odot}$. Recently, by using the radioactive tail luminosity, Afsariardchi et al. (2020) estimated mean $M_{56 \mathrm{Ni}}$ values of $0.06,0.11,0.20$, and $0.15 \mathrm{M}_{\odot}$ for $\mathrm{SNe} \mathrm{IIb}, \mathrm{Ib}$, Ic, and Ic-BL, respectively ${ }^{9}$. Inserting these values in equation (33) we get $f_{\mathrm{SE}}^{\mathrm{Ni}}=0.116 \mathrm{M}_{\odot}$, which is similar to the previous finding. Since the SN samples of Anderson (2019) and Afsariardchi et al. (2020) are not corrected for selection bias, we adopt $f_{\mathrm{SE}}^{\mathrm{Ni}}<0.12 \mathrm{M}_{\odot}$

Replacing the $f_{\mathrm{SE}}^{\mathrm{Ni}}$ and $f_{\mathrm{II}}^{\mathrm{Ni}}$ values in equation (32), we get $f_{\mathrm{CC}}^{\mathrm{Ni}}<0.064 \mathrm{M}_{\odot}$, where the contribution of normal SNe II to $f_{\mathrm{CC}}^{\mathrm{Ni}}$ is $>36$ per cent. Finally, if we assume $\eta=1.07$ for all CC SN subtypes, then from equation (21) we obtain $\bar{y}_{\mathrm{Fe}}<0.068 \mathrm{M}_{\odot}$ for CC SNe.

\subsection{Steepness as ${ }^{56} \mathrm{Ni}$ mass indicator}

From the analysis of nine normal SNe II and the longrising SN 1987A, Elmhamdi et al. (2003b) reported a linear correlation between $\log M_{56} \mathrm{Ni}$ and the maximum value of $d V / d t$ during the transition phase, called $V$-band steepness $S_{V}$. This correlation was also rebuilt by Singh et al. (2018), which included another 30 SNe to the sample of Elmhamdi et al. (2003b). The observed correlation is proposed to be a consequence of the ${ }^{56} \mathrm{Ni}$ heating during the transition phase (e.g. Pumo \& Zampieri 2011, 2013). The latter produces slower transitions of the luminosity from the end of the plateau phase to the beginning of the radioactive tail as $M_{56} \mathrm{Ni}$ increases. Since the correlation between $\log M_{56} \mathrm{Ni}$ and steepness has not been studied for bands other than $V$, in this work we will include the grRiI bands in the analysis.

In order to measure the $x$-band steepness, $S_{x}$, we represent the light-curve transition phase by the function

$m_{x}(t)=m_{0, x}-\frac{a_{0, x}}{1+e^{\left(t-t_{\mathrm{PT}, x}\right) / w_{0, x}}}+p_{0, x} \frac{t-t_{\mathrm{PT}, x}}{100 \mathrm{~d}}$

(e.g. Olivares E. et al. 2010; Valenti et al. 2016). The parameters $m_{0, x}, a_{0, x}, t_{\mathrm{PT}, x}$ (the middle of the transition phase), $w_{0, x}$, and $p_{0, x}$ are obtained maximizing the model log-likelihood (equation C1). Fig. 16 shows this analytical fit applied to the $V$-band photometry of SN 2014G. Using the aforementioned parametric function, the $x$-band steepness (in $\mathrm{mag} \mathrm{d}^{-1}$ ) in the $\mathrm{SN}$ rest frame is given by

$S_{x}=\left(\frac{a_{0, x}}{4 w_{0, x}}+\frac{p_{0, x}}{100 \mathrm{~d}}\right) /\left(1+z_{\text {helio }}^{\mathrm{SN}}\right)$.

Columns 2-7 of Table L7 list the $g V r R i I$-band $S_{x}$ values and their bootstrap errors. As visible in the table, the $S_{x}$ estimates are in general not available for each of the $g V r R i I$ bands. In those cases we estimate $S_{x}$ indirectly from the steepnesses in bands other than $x$ (see Appendix J). These indirect $S_{x}$ values $\left(S_{x}^{*}\right)$ for the $g$ VrRiI bands and their errors are in Columns $8-13$ of Table L7. Table 6 lists the mean and $\hat{\sigma}$ values of the $S_{x}^{*}-S_{x}$ estimates for the $g$ VrRiI bands.

9 Mean values were computed with $\leq 8$ SNe per subtype, so we caution that those values are not statistically significant.

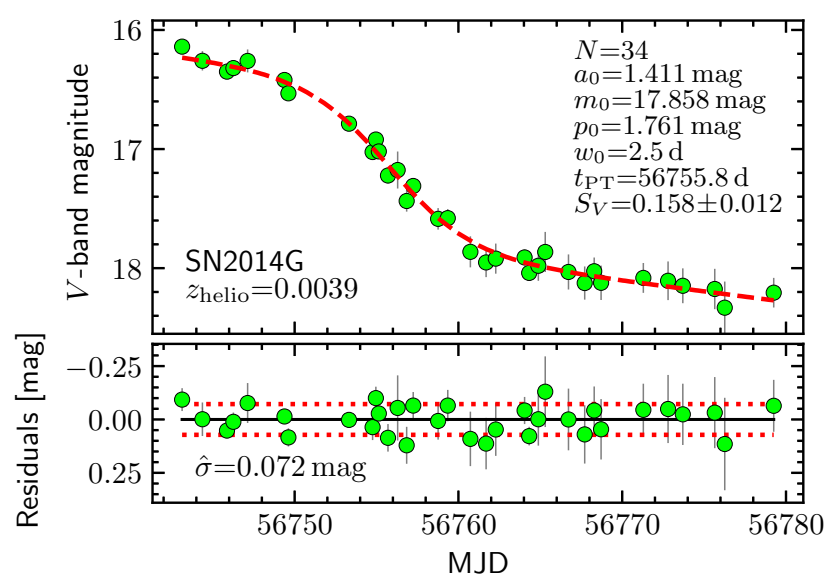

Figure 16. Top panel: $V$-band light curve of SN 2014G in the transition phase, where the dashed line corresponds to the best fit. Bottom panel: best fit residuals, where dotted lines indicate the $\pm 1 \hat{\sigma}$ limits. Error bars are $1 \sigma$ errors.

Table 6. Mean $S_{x}^{*}-S_{x}$ values.

\begin{tabular}{cccccccc}
\hline$x$ & $\left\langle S_{x}^{*}-S_{x}\right\rangle$ & $\hat{\sigma}$ & $N$ & $x$ & $\left\langle S_{x}^{*}-S_{x}\right\rangle$ & $\hat{\sigma}$ & $N$ \\
\hline$g$ & -0.002 & 0.016 & 16 & $V$ & -0.003 & 0.024 & 41 \\
$r$ & 0.003 & 0.029 & 20 & $R$ & -0.002 & 0.017 & 32 \\
$i$ & -0.004 & 0.036 & 22 & $I$ & 0.002 & 0.018 & 31 \\
\hline \multicolumn{7}{l}{ Note. Mean and $\hat{\sigma}$ values are in mag d ${ }^{-1}$ units. }
\end{tabular}

Table 7. $\log M_{56 \mathrm{Ni}}$ versus $S_{x}$ calibrations.

\begin{tabular}{|c|c|c|c|c|c|}
\hline$x$ & $\begin{array}{c}c_{0, x} \\
(\mathrm{dex})\end{array}$ & $\begin{array}{c}c_{1, x} \\
(\operatorname{dex})\end{array}$ & $\begin{array}{c}\hat{\sigma} \\
(\mathrm{dex})\end{array}$ & $N$ & $r_{x}$ \\
\hline$g$ & $-1.219 \pm 0.049$ & $-1.844 \pm 0.256$ & 0.217 & 72 & -0.67 \\
\hline$V$ & $-1.201 \pm 0.043$ & $-1.789 \pm 0.183$ & 0.210 & 72 & -0.69 \\
\hline$r$ & $-1.238 \pm 0.047$ & $-2.130 \pm 0.292$ & 0.219 & 72 & -0.66 \\
\hline$R$ & $-1.217 \pm 0.042$ & $-2.053 \pm 0.217$ & 0.210 & 72 & -0.69 \\
\hline$i$ & $-1.237 \pm 0.044$ & $-1.901 \pm 0.231$ & 0.221 & 72 & -0.65 \\
\hline$I$ & $-1.223 \pm 0.045$ & $-2.106 \pm 0.253$ & 0.219 & 72 & -0.66 \\
\hline
\end{tabular}

The mean values are statistically consistent with zero within $1 \hat{\sigma} / \sqrt{N}$. Therefore, for SNe without an specific $S_{x}$ value, we can use their respective $S_{x}^{*}$ as a proxy.

Fig. 17 shows $\log M_{56_{\mathrm{Ni}}}$ versus $S_{x}$ for the $g \operatorname{VrRiI}$ bands. Through the model selection procedure (Appendix C), we find that the correlation between $\log M_{56_{\mathrm{Ni}}}$ and $S_{x}$ is well represented by the straight line

$\log M_{56 \mathrm{Ni}}=c_{0, x}+c_{1, x} S_{x}$

which, for the $V$-band, corresponds to the best fit proposed by Elmhamdi et al. (2003b). The parameters $c_{0, x}$ and $c_{1, x}$ (and their bootstrap errors) for the $g \operatorname{VrRiI}$ bands are reported in Table 7 . To evaluate the linear correlation between $\log M_{56} \mathrm{Ni}$ and $S_{x}$, we calculate the Pearson correlation coefficient $r_{x}$, listed in Column 6 of Table 7 . The probability of obtaining $\left|r_{x}\right| \geq 0.65$ from a random population with $N=72$ is $<0.001$ per cent.

The observed $\hat{\sigma}$ of $0.21-0.22$ dex for the $g \operatorname{VrRiI}$ bands indicates that there is no preferred band for the $\log M_{56} \mathrm{Ni}$ versus $S_{x}$ correlation. Since the $\hat{\sigma}$ values are $\sim 0.17 \mathrm{dex}$ greater in quadrature than the typical random error 

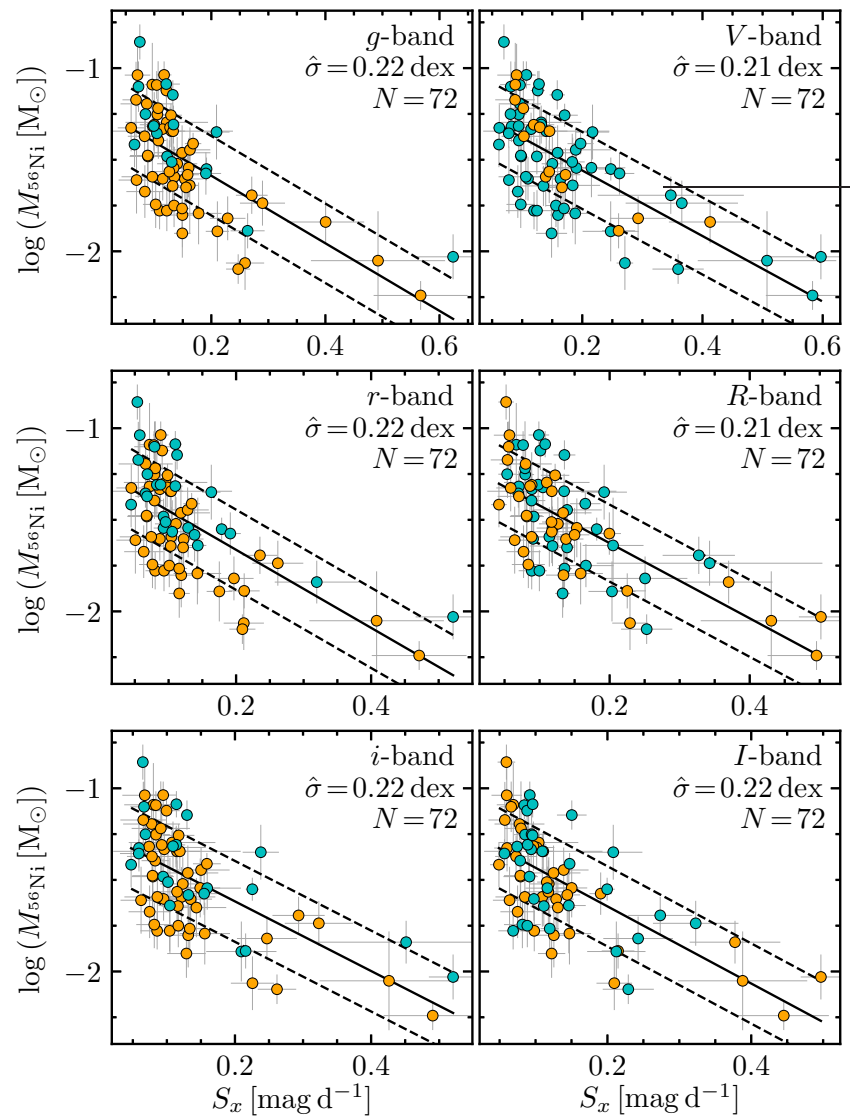

Figure 17. $\log M_{56} \mathrm{Ni}$ against $S_{x}$ for $g V r R i I$ bands. Cyan circles are $\mathrm{SNe}$ with $S_{x}$ measured from their $x$-band light curves, while orange circles are $\mathrm{SNe}$ with $S_{x}$ estimated from the steepnesses in other bands. Solid lines are straight-line fits to the data, dashed lines are $\pm 1 \hat{\sigma}$ limits around the fits, and error bars are $1 \sigma$ errors.

( 0.13 dex $)$, the observed dispersion is mainly intrinsic. The latter was also pointed out by Pumo \& Zampieri (2013). Indeed, the shape of light curves in the transition phase not only depends on $M_{56} \mathrm{Ni}$ but also, among others, on the $\mathrm{H}$ mass retained before the explosion and the ${ }^{56} \mathrm{Ni}$ mixing (e.g. Young 2004; Bersten et al. 2011; Kozyreva et al. 2019).

Fig. 18 shows the residuals of the $\log M_{56 \mathrm{Ni}}$ versus $S_{V}$ correlation (i.e. upper-right panel of Fig. 17) plotted against $M_{V}^{50 \mathrm{~d}}$, where we detect a linear dependence of the residuals on $M_{V}^{50 \mathrm{~d}}$. Therefore the Elmhamdi et al. (2003b) relation over and underestimates the $\log M_{56} \mathrm{Ni}$ of sub-luminous and moderately-luminous SNe II, respectively, by up to $\sim 0.3$ dex. This fact, along with the low statistical precision of the Elmhamdi et al. (2003b) relation to measure $M_{56 \mathrm{Ni}}$ (around 50 per cent), makes the latter method poorly suited for ${ }^{56} \mathrm{Ni}$ mass measurements.

\subsection{Nickel-magnitude-steepness relation}

Our previous finding suggests a correlation of $\log M_{56 \mathrm{Ni}}$ as a function not only of $S_{x}$ but also of the $x$-band absolute magnitude at $\Delta t=50 \mathrm{~d}\left(M_{x}^{50 \mathrm{~d}}\right)$. The $M_{x}^{50 \mathrm{~d}}$ values for $\mathrm{grRiI}$ are listed in Table L9. If $M_{x}^{50 \mathrm{~d}}$ is not available for a given band, then we estimate it using photometry in other bands and magnitude transformation formulae (see Appendix $\mathrm{H}$ ).

Using the model selection procedure (Appendix C), we

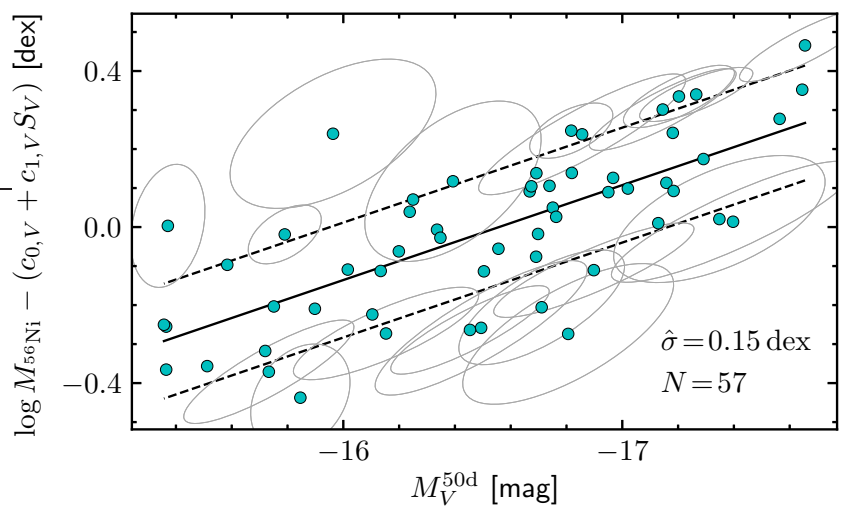

Figure 18. Residuals of the $\log M_{56 \mathrm{Ni}}$ versus $S_{V}$ correlation, as a function of $M_{V}^{50 d}$. The solid and dashed lines have the same meaning than in Fig 17. Ellipses are $1 \sigma$ confidence regions, which for clarity are drawn only for $\mathrm{SNe}$ outside the dashed lines.

find that the correlation of $\log M_{56}{ }_{\mathrm{Ni}}$ as a function of $S_{x}$ and $M_{x}^{50 \mathrm{~d}}$ can be represented by

$\log M_{56 \mathrm{Ni}}=a_{x}+b_{x} M_{x}^{50 \mathrm{~d}}+c_{x} \log S_{x}$,

where $\log S_{x}$ is given by equation (E3). Fig. 19 shows the nickel-magnitude-steepness (NMS) relation for the $g V r R i I$ bands, while Table 8 lists the parameters (and their bootstrap errors) of equation (38) along with the ranges of $M_{x}^{50 \mathrm{~d}}$ and $\log S_{x}$ where the relation is valid. To evaluate the linear correlation of $\log M_{56 \mathrm{Ni}}$ on $M_{x}^{50 \mathrm{~d}}$ and $\log S_{x}$, we calculate the multiple correlation coefficient $\left(R_{x}^{\mathrm{mc}}\right.$, reported in Column 10 of Table 8). The probability of obtaining $R_{x}^{\mathrm{mc}} \geq 0.89$ from a random population is $<0.001$ per cent.

The NMS relation allows to measure $\log M_{56 \mathrm{Ni}}$ with a statistical precision of $0.12-0.14 \mathrm{dex}\left(M_{56} \mathrm{Ni}\right.$ error of $\sim 30$ per cent). The observed random error is about $0.08 \mathrm{dex}$, so the intrinsic random error on the NMS relation $\left(\sigma_{0, x}\right.$, listed in Column 6 of Table 8) is around 0.10 dex. Since $\sim 80$ per cent of the SNe used to calibrate equation (38) have $\log M_{56_{\mathrm{Ni}}}$ computed with $I$ - or $i$-band photometry, we adopt a systematic uncertainty due to the $\mathrm{ZP}^{\mathrm{BC}}$ errors of $0.044 \mathrm{dex}$ (the average between the errors on $\mathrm{ZP}_{I}^{\mathrm{BC}}$ and $\mathrm{ZP}_{i}^{\mathrm{BC}}$ in dex scale). The total systematic error $\left(\sigma_{\text {sys }}\right)$, including the uncertainty due to $\alpha$, is of $0.047 \mathrm{dex}$, while the total error on $\log M_{56} \mathrm{Ni}$ provided by the NMS relation is given by

$\sigma_{\log M_{56_{\mathrm{Ni}}}}=\sqrt{\left(b_{x} \sigma_{M_{x}^{50 \mathrm{~d}}}\right)^{2}+\left(c_{x} \sigma_{\log S x}\right)^{2}+\sigma_{0, x}^{2}+\sigma_{\mathrm{sys}}^{2}}$.

Since $\left|b_{x}\right|<0.4$, the $\log M_{56_{\mathrm{Ni}}}$ values estimated with the NMS relation are less dependent on $E_{B-V}^{\mathrm{h}}$ and $\mu$ than those computed with the radioactive tail photometry and the $\mathrm{BC}$ technique (Appendix D).

As a first application of the NMS relation, we compute $\log M_{56 \mathrm{Ni}}$ estimates $\left(\log M_{56 \mathrm{Ni}}^{\mathrm{NMS}}\right.$ ) for a sample of normal SNe II observed by the Zwicky Transient Facility (ZTF; Bellm et al. 2019; Graham et al. 2019). Specifically, we employ SNe from the ZTF bright transient survey ${ }^{10}$ (BTS; Fremling et al. 2020; Perley et al. 2020), which consists on SNe brighter than 19 mag. From this magnitude-limited survey, we select SNe spectroscopically classified as SNe II, discarding those (1) classified as Type IIb or IIn, (2) with long-

10 https://sites.astro.caltech.edu/ztf/bts/explorer.php 

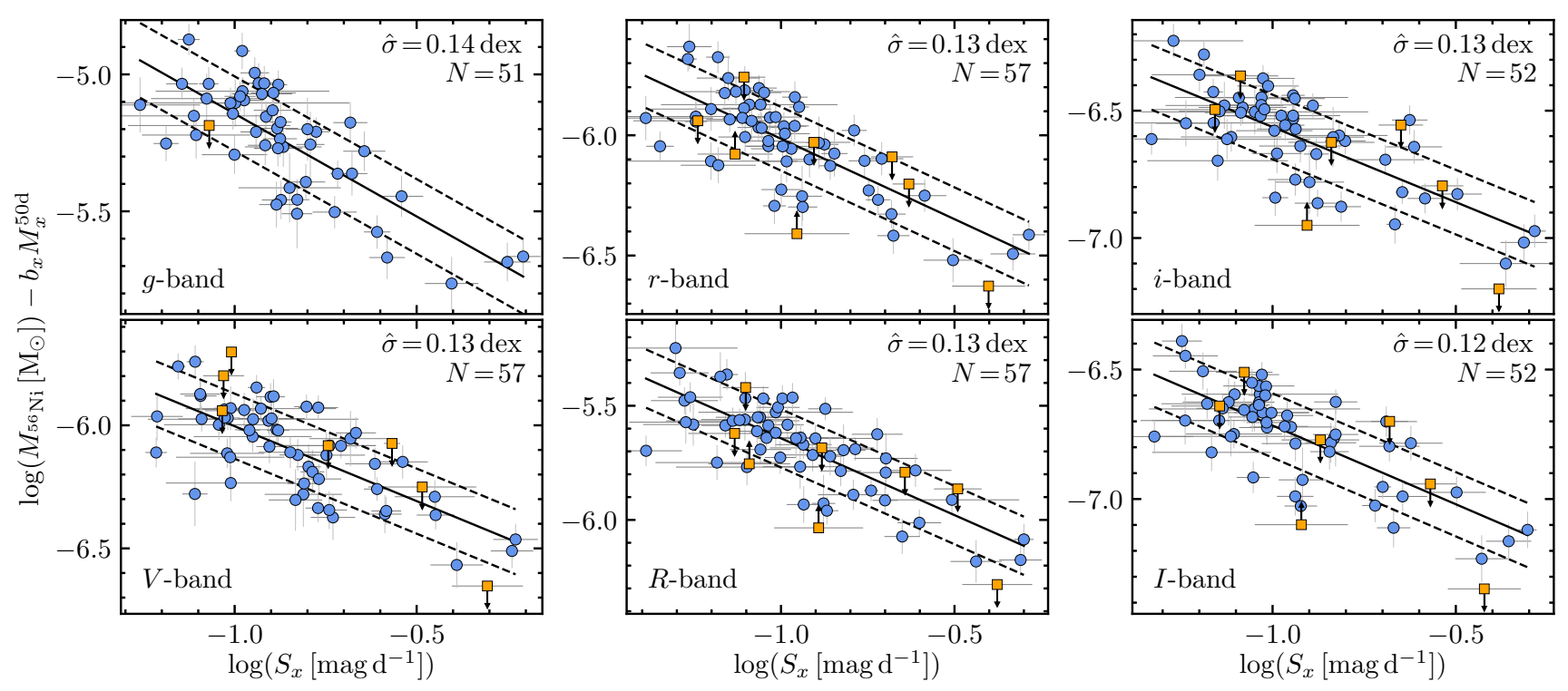

Figure 19. NMS relations for the $g V r R i I$ bands. Solid lines are the best fits to the data (circles), while dashed lines indicate the $\pm 1 \hat{\sigma}$ limits around the fits. Orange squares correspond to SNe with lower/upper limits on $M_{x}^{50 \mathrm{~d}}$. Error bars are $1 \sigma$ errors.

Table 8. NMS relation parameters.

\begin{tabular}{|c|c|c|c|c|c|c|c|c|c|}
\hline$x$ & $\begin{array}{c}a_{x} \\
(\mathrm{dex})\end{array}$ & $\begin{array}{c}b_{x} \\
\left(\operatorname{dex~mag}^{-1}\right)\end{array}$ & $c_{x}$ & $\begin{array}{c}\hat{\sigma} \\
(\operatorname{dex})\end{array}$ & $\begin{array}{l}\sigma_{0, x} \\
(\mathrm{dex})\end{array}$ & $N$ & $\begin{array}{c}M_{x}^{50 \mathrm{~d}} \text { range } \\
(\mathrm{mag})\end{array}$ & $\begin{array}{c}\log S_{x} \text { range } \\
\text { (dex) }\end{array}$ & $R_{x}^{\mathrm{mc}}$ \\
\hline$g$ & $-5.894 \pm 0.461$ & $-0.2311 \pm 0.0306$ & $-0.750 \pm 0.097$ & 0.136 & 0.112 & 51 & $-14.9,-17.4$ & $-1.26,-0.21$ & 0.89 \\
\hline$V$ & $-6.612 \pm 0.409$ & $-0.2778 \pm 0.0266$ & $-0.608 \pm 0.083$ & 0.132 & 0.106 & 57 & $-15.4,-17.7$ & $-1.22,-0.23$ & 0.89 \\
\hline$r$ & $-6.683 \pm 0.459$ & $-0.2710 \pm 0.0294$ & $-0.669 \pm 0.087$ & 0.132 & 0.107 & 57 & $-15.6,-18.1$ & $-1.39,-0.29$ & 0.90 \\
\hline$R$ & $-6.315 \pm 0.449$ & $-0.2487 \pm 0.0287$ & $-0.673 \pm 0.082$ & 0.128 & 0.094 & 57 & $-15.7,-18.2$ & $-1.39,-0.30$ & 0.90 \\
\hline$i$ & $-7.154 \pm 0.442$ & $-0.3066 \pm 0.0281$ & $-0.590 \pm 0.079$ & 0.127 & 0.098 & 52 & $-15.4,-17.7$ & $-1.33,-0.28$ & 0.90 \\
\hline$I$ & $-7.324 \pm 0.430$ & $-0.3083 \pm 0.0267$ & $-0.608 \pm 0.080$ & 0.124 & 0.098 & 52 & $-15.7,-18.1$ & $-1.32,-0.30$ & 0.91 \\
\hline
\end{tabular}

Notes. $\log M_{56 \mathrm{Ni}}=a_{x}+b_{x} M_{x}^{50 \mathrm{~d}}+c_{x} \log S_{x} . M_{56 \mathrm{Ni}}, M_{x}^{50 \mathrm{~d}}$, and $S_{x}$ are in units of $\mathrm{M}_{\odot}, \mathrm{mag}$, and $\operatorname{mag} \mathrm{d}^{-1}$, respectively.

rising light curves, (3) with less than three $r_{\mathrm{ZTF}}$ photometric points in the radioactive tail, (4) with $t_{0}$ errors greater than $10 \mathrm{~d}$, and (5) with absolute magnitudes and steepnesses outside the range where the NMS relation is valid. The selected ZTF BTS (SZB) sample of 28 normal SNe II and their main properties are summarized in Table L10, while Fig. 20 shows their $r_{\mathrm{ZTF}}$ light curves $^{11}$. Out of the SNe in the SZB set, 24 have $M_{R}^{\max }<-17 \mathrm{mag}$, so the sample consists mainly of moderately-luminous SNe II.

Given that $r_{\mathrm{ZTF}}-R=0.14 \mathrm{mag}$ from the photospheric to the radioactive tail phase (see Appendix $\mathrm{H}$ ), for the $r_{\text {ZTF }}$ band we adopt the $R$-band NMS relation, but with $a_{r_{\mathrm{ZTF}}}=a_{R}-0.14 b_{R}$ equal to $-6.281 \mathrm{dex}$. Since we cannot measure $E_{B-V}^{\mathrm{h}}$ for the SZB sample with the available data, we assume $E_{B-V}^{\mathrm{h}}=0.16 \pm 0.15 \mathrm{mag}$ (the average of the $E_{B-V}^{\mathrm{h}}$ distribution shown in Fig. 6). Column 9 of Table L10 lists the inferred $\log M_{56}^{\mathrm{NMS}}$ values. For comparison, we also compute $\log M_{56} \mathrm{Ni}$ with the radioactive tail luminosity ( $\log M_{56 \mathrm{Ni}}^{\text {tail }}$, Column 10 of Table L10), using a constant $\mathrm{BC}_{r_{\mathrm{ZTF}}}=\mathrm{BC}_{R}-0.14$ equivalent to $11.93 \pm 0.14 \mathrm{mag}$.

The mean offset between the $\log M_{56 \mathrm{Ni}}^{\mathrm{tail}}$ and $\log M_{56 \mathrm{Ni}}^{\mathrm{NMS}}$ values is of 0.038 dex with a $\hat{\sigma}$ of 0.110 dex. The $\hat{\sigma}$ value is similar to that expected for the NMS relation $(0.13 \mathrm{dex})$,

11 Photometry obtained from the ALeRCE (Förster et al. 2021) website (https://alerce.online/). while the offset is consistent with zero within $1.9 \hat{\sigma} / \sqrt{N}$. As stated in Section 4.2.2, the use of the constant $\mathrm{BC}_{R}$ reported in Table $2\left(\mathrm{BC}_{R}=12.07\right)$ overestimates the radioactive tail luminosities (and therefore the $\log M_{56 \mathrm{Ni}}^{\mathrm{tail}}$ values) of moderately-luminous SNe II. To roughly estimate a more appropriate constant $\mathrm{BC}_{R}$ for the $\mathrm{SZB}$ sample, we use the $\mathrm{ZP}_{R}^{\mathrm{SN}}$ values of the nine $\mathrm{SNe}$ in the $\mathrm{BC}$ calibration set with $M_{R}^{\max }<-17 \mathrm{mag}$, obtaining a $\mathrm{BC}_{R}$ of 12.13 mag. Increasing $\mathrm{BC}_{r_{\mathrm{ZTF}}}$ by $0.06 \mathrm{mag}$ decreases the offset from 0.046 to 0.014 dex, being consistent with zero within $0.7 \hat{\sigma} / \sqrt{N}$. In addition, the offset decreases from $1.9 \hat{\sigma} / \sqrt{N}$ to zero if we adopt $E_{B-V}^{\mathrm{h}}=0.05 \mathrm{mag}$. Therefore, part of the offset could be due to an overestimation of the adopted $E_{B-V}^{\mathrm{h}}$. The results we obtain with the SZB sample provides further evidence supporting the usefulness of the NMS relation for $\log M_{56 \mathrm{Ni}}$ measurements.

\section{DISCUSSION}

\subsection{Comparison with other works}

Table 2 summarizes the $\mathrm{BC}$ values for $\mathrm{SNe}$ II in the radioactive tail reported by Hamuy (2001), Bersten \& Hamuy (2009), Maguire et al. (2010), and Pejcha \& Prieto (2015a). From each value we subtract the zero-point used to define the apparent bolometric magnitude scale 


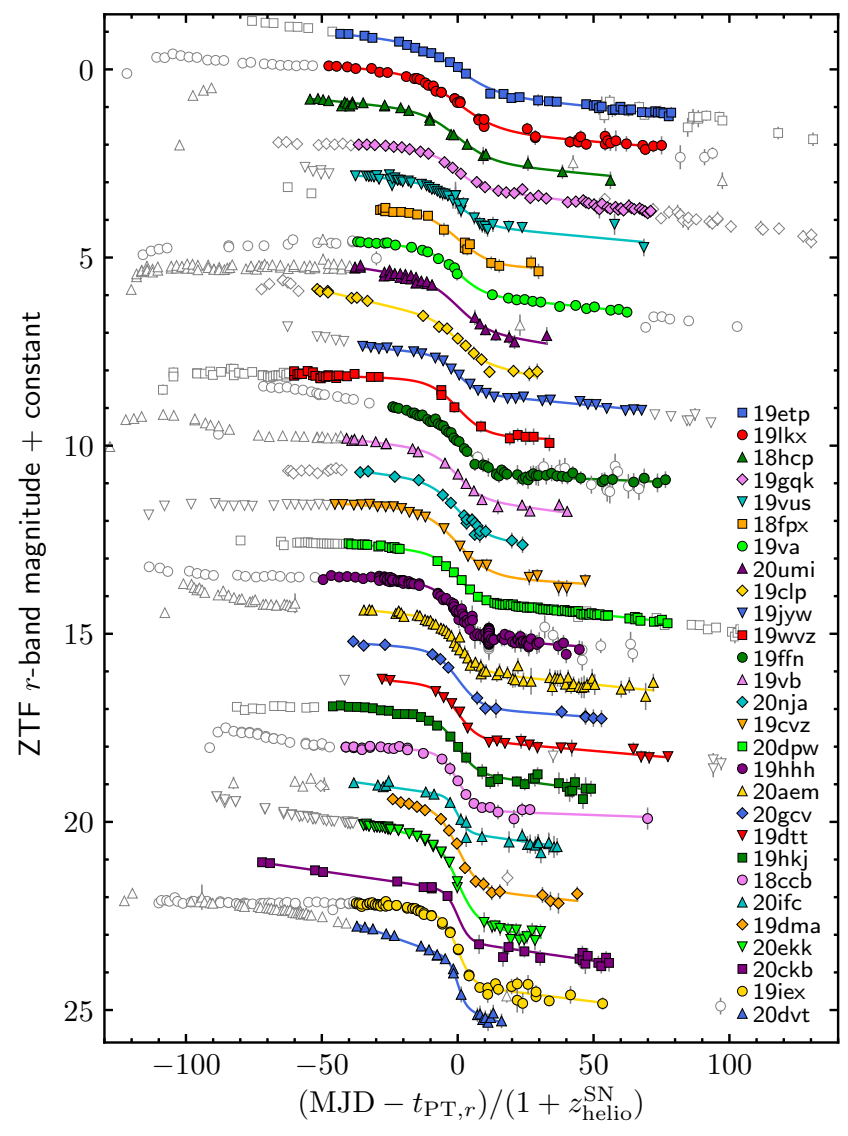

Figure 20. $r_{\text {ZTF }}$ light curves of the 28 normal SNe II in the SZB sample. Data used to measure the steepness are shown with colour-filled symbols, while solid lines are analytical fits.

(-10.89 mag for Hamuy 2001 and Maguire et al. 2010, -11.64 mag for Bersten \& Hamuy 2009, and -11.48 mag for Pejcha \& Prieto 2015a). Since previous works reported constant $\mathrm{BC}$ values, for the comparison we use our estimates assuming a constant $\mathrm{BC}$, which are also listed in the table. The $\mathrm{BC}$ values reported in the literature are consistent with our estimations within $\pm 1.1 \sigma$. Except for Pejcha \& Prieto (2015a), which did not report BC errors, the uncertainties provided in previous works are lower than those reported here. The latter is due to the few SNe used in previous studies to compute BCs. Indeed, they used only SN 1999em and the long-rising SN 1987A to compute BCs. It is interesting to note the good agreement between our constant $\mathrm{BC}$ values for rRiI bands and those of Pejcha \& Prieto (2015a) within $\pm 0.04 \mathrm{mag}$

Table 10 collects the mean and $\hat{\sigma}$ values of the normal SN II $M_{56} \mathrm{Ni}$ distributions presented in Blanc \& Greggio (2008) and Müller et al. (2017). Despite the Blanc \& Greggio (2008) sample includes the long-rising SN 1987A, we find that removing that SN only marginally modifies the reported mean and $\hat{\sigma}$ value. In addition, we include the mean ${ }^{56} \mathrm{Ni}$ mass computed with the 107 normal SNe II in the sample of Anderson $(2019)^{12}$. Since pre-

$12 M_{56} \mathrm{Ni}$ values are reported in Meza \& Anderson (2020), from which we remove SN 2007od, the long-rising SNe 1987A, 1998A, $2000 \mathrm{cb}, 2006 \mathrm{~V}, 2006 \mathrm{au}$, and 2009E, and the LLEV SN 2008bm.
Table 9. Constant $\mathrm{BC}$ values for the radioactive tail.

\begin{tabular}{cccl}
\hline $\mathrm{BC}_{x}(\mathrm{mag})$ & $x$ & $N$ & Reference \\
\hline $11.15 \pm 0.06$ & $V$ & $2^{a}$ & Hamuy (2001) \\
$10.94 \pm 0.05$ & $V$ & $1^{a}$ & Bersten \& Hamuy (2009) \\
$11.22 \pm 0.06$ & $V$ & $2^{a}$ & Maguire et al. (2010) \\
11.27 & $V$ & $26^{b}$ & Pejcha \& Prieto (2015a) \\
$11.15 \pm 0.18$ & $V$ & 15 & This work \\
11.90 & $r$ & $26^{b}$ & Pejcha \& Prieto (2015a) \\
$11.89 \pm 0.16$ & $r$ & 15 & This work \\
12.07 & $R$ & $26^{b}$ & Pejcha \& Prieto (2015a) \\
$12.07 \pm 0.14$ & $R$ & 15 & This work \\
11.95 & $i$ & $26^{b}$ & Pejcha \& Prieto (2015a) \\
$11.91 \pm 0.12$ & $i$ & 15 & This work \\
12.41 & $I$ & $26^{b}$ & Pejcha \& Prieto (2015a) \\
$12.44 \pm 0.11$ & $I$ & 15 & This work \\
\hline${ }^{a}$ It includes the long-rising SN 1987A. \\
${ }^{b}$ Only six SNe with optical and near-IR photometry \\
in the radioactive tail.
\end{tabular}

Table 10. Mean ${ }^{56} \mathrm{Ni}$ mass values for normal SNe II.

\begin{tabular}{cccccl}
\hline $\begin{array}{c}\left\langle M_{56 \mathrm{Ni}}\right\rangle^{\text {unc }} \dagger \\
\left(\mathrm{M}_{\odot}\right)\end{array}$ & $\begin{array}{c}\hat{\sigma} \\
\left(\mathrm{M}_{\odot}\right)\end{array}$ & $N$ & $\begin{array}{c}\hat{\sigma} / \sqrt{N} \\
\left(\mathrm{M}_{\odot}\right)\end{array}$ & $N_{c}{ }^{*}$ & Reference $^{\ddagger}$ \\
\hline 0.066 & 0.082 & 28 & 0.015 & 17 & B08 \\
0.046 & 0.048 & 38 & 0.008 & 33 & M17 \\
0.042 & 0.044 & 107 & 0.004 & 75 & A19 \\
0.042 & 0.033 & 109 & 0.003 & - & This work \\
\hline
\end{tabular}

${ }^{\dagger}\left\langle M_{56 \mathrm{Ni}}\right\rangle$ uncorrected for selection bias.

* Number of SNe in common with our full sample (109 SNe)

¥B08: Blanc \& Greggio (2008); M17: Müller et al. (2017);

A19: Anderson (2019), selecting only normal SNe II.

vious estimates are not corrected for selection bias, for the comparison we use our $\left\langle M_{56 \mathrm{Ni}}\right\rangle^{\text {unc }}$ value. The latter value and those from the aforementioned samples are consistent within $1.6 \hat{\sigma} / \sqrt{N}$. It is worth mentioning that the collected $\left\langle M_{56_{\mathrm{Ni}}}\right\rangle^{\text {unc }}$ values are not independent since they were computed with SN samples having objects in common. The number of SNe in common between a given set and our full sample is indicated in Column 5. In particular, the similarity between our result and that obtained from the Anderson (2019) sample is because both analyses have 70 per cent of $\mathrm{SNe}$ in common.

We also compare the $\log M_{56 \mathrm{Ni}}$ values of the $\mathrm{SNe}$ in common between our SN set and the samples analysed in Müller et al. (2017), Valenti et al. (2016), and Sharon \& Kushnir (2020). Müller et al. (2017) employed the methodology of Pejcha \& Prieto (2015a), which computes $\log M_{56 \mathrm{Ni}}$ using the luminosity at $\Delta t=200 \mathrm{~d}$ and equation (3) of Hamuy (2003). Valenti et al. (2016) used

$M_{56_{\mathrm{Ni}}} / \mathrm{M}_{\odot}=0.075 L_{\mathrm{SN}}^{\mathrm{opt}}(\Delta t) / L_{87 \mathrm{~A}}^{\mathrm{opt}}(\Delta t)$,

being $L_{\mathrm{SN}}^{\mathrm{opt}}(\Delta t)$ and $L_{87 \mathrm{~A}}^{\mathrm{opt}}(\Delta t)$ the optical quasi-bolometric luminosity in the radioactive tail of a specific $\mathrm{SN}$ and of the long-rising SN 1987A, respectively. Sharon \& Kushnir (2020) used the radioactive tail luminosity and the set of equations presented in Wygoda et al. (2019) to derive $\log M_{56_{\mathrm{Ni}}}$. For each sample we compute the differences between its $\log M_{56_{\mathrm{Ni}}}$ measurements, and calculate the mean offset $(\Delta)$ and its $\hat{\sigma}$. Then, to track the main source of the observed dispersion, we repeat the previous process, but recomputing our $\log M_{56} \mathrm{Ni}$ values without correcting for the $\gamma$-ray leakage (except for Sharon \& Kushnir 2020, which in- 
Table 11. Mean $\log M_{56}{ }_{\mathrm{Ni}}$ differences between different works.

\begin{tabular}{lcrc}
\hline $\log _{56}{ }_{\text {Ni }}$ differences $^{\dagger}$ & $N$ & $\Delta$ (dex) & $\hat{\sigma}($ dex $)$ \\
\hline M17-here & 33 & -0.01 & 0.29 \\
M17-here $(\mathrm{a})$ & 33 & 0.01 & 0.28 \\
M17-here(a,b) & 33 & 0.07 & 0.13 \\
M17-here(a,b,c) & 33 & 0.05 & 0.08 \\
V16-here & 33 & -0.15 & 0.30 \\
V16-here (a,b,c) & 33 & -0.05 & 0.14 \\
S20-here & 7 & -0.09 & 0.15 \\
S20-here (b,c) & 7 & 0.02 & 0.05 \\
\hline
\end{tabular}

${ }^{\dagger}$ M17: Müller et al. (2017); S20: Sharon \& Kushnir (2020); V16: Valenti et al. (2016); here: this work, uncorrected for $\gamma$ ray leakage (a), and using the distance moduli (b) and colour excesses (c) of the comparison work.

cluded this correction) and using the $\mu$ and $E_{B-V}^{\mathrm{h}}$ values of the comparison work. The $\Delta$ and $\hat{\sigma}$ values are listed in Table 11.

From the comparison with the Müller et al. (2017) sample we obtain $\hat{\sigma}=0.29 \mathrm{dex}$. This value decreases to $0.28 \mathrm{dex}$ when we do not correct for $\gamma$-ray leakage, to 0.13 dex when we use the distance moduli of Müller et al. (2017), and to 0.08 dex if we also use their colour excesses. This indicates that differences between our $\log M_{56} \mathrm{Ni}$ estimates and those of Müller et al. (2017) are mainly due to differences in the adopted distance moduli and colour excesses. Therefore, the $\hat{\sigma}$ value of 0.08 dex represents the typical $\log M_{56 \mathrm{Ni}}$ error for single $\mathrm{SNe}$ due to differences in the methodology used to compute $\log M_{56} \mathrm{Ni}$. For the other two comparison samples we arrive at similar results.

The $\Delta$ value from the comparison with the sample of Müller et al. (2017) is equivalent to $3.5 \hat{\sigma} / \sqrt{N}$, which indicates the presence of a systematic offset. We note that, of the 0.05 dex offset, 0.03 dex is due to the numerical coefficients of the equation used in Pejcha \& Prieto (2015a) to compute $\log M_{56_{\mathrm{Ni}}}{ }^{13}$. The remaining 0.02 dex is statistically consistent with zero within $1.4 \hat{\sigma} / \sqrt{N}$. The $\Delta$ values from the comparison with the samples of Valenti et al. (2016) and Sharon \& Kushnir (2020) are statistically consistent with zero within 2.0 and $1.0 \hat{\sigma} / \sqrt{N}$, respectively.

\subsection{Systematics}

The models we use in this work (SN II spectra and nucleosynthesis yields) were generated by adopting many approximations and assumptions that help to characterize the underlying complex physical processes of SN explosions. Therefore, our $\mathrm{ZP}_{x}^{\mathrm{BC}}, \log M_{56 \mathrm{Ni}},\left\langle M_{56 \mathrm{Ni}}\right\rangle$, and $\bar{y}_{\mathrm{Fe}}$ values are potentially affected by systematics on the spectral models, while $\bar{y}_{\mathrm{Fe}}$ is also affected by systematics on nucleosynthesis yield models. An analysis and quantification of those systematics is beyond the scope of this study.

\subsubsection{Local Hubble-Lemaître constant}

The distance moduli of $92 \mathrm{SNe}$ in our sample were estimated as the weighted average of $\mu_{\mathrm{TF}}, \mu_{\mathrm{HLL}}$, and $\mu_{\mathrm{SVF}}$ (see Sec-

13 Equation (3) of Hamuy (2003) is not accurate. To estimate $\log M_{56 \mathrm{Ni}}$ we recommend the set of equations presented in Wygoda et al. (2019). tion 3.6.1). The latter are anchored to Cepheid-calibrated $H_{0}$ values of around $75 \mathrm{~km} \mathrm{~s}^{-1} \mathrm{Mpc}^{-1}$. If we adopt a TRGBcalibrated $H_{0}$ value between 69.6 and $72.4 \mathrm{~km} \mathrm{~s}^{-1} \mathrm{Mpc}^{-1}$ (e.g. Freedman et al. 2020; Yuan et al. 2019), then the $\mu$ values of our SN sample increase by about $0.08-0.16 \mathrm{mag}$. In this case, we obtain $\left\langle M_{56 \mathrm{Ni}}\right\rangle$ values around $0.040-0.044 \mathrm{M}_{\odot}$. If we assume that the true local $H_{0}$ value lies between 71 and $75 \mathrm{~km} \mathrm{~s}^{-1} \mathrm{Mpc}^{-1}$ with a uniform probability, then the systematic offset in $\left\langle M_{56} \mathrm{Ni}\right\rangle$ induced by the $H_{0}$ uncertainty ranges between 0.0 and $0.005 \mathrm{M}_{\odot}$, with a mean of $0.002 \pm 0.001 \mathrm{M}_{\odot}$. This systematic error is lower than that induced by the $\mathrm{ZP}^{\mathrm{BC}}$ error $\left(0.003 \mathrm{M}_{\odot}\right)$. Therefore the $H_{0}$ uncertainty is not so relevant for our current analysis.

\subsubsection{Early dust formation}

To compute $\log M_{56}{ }_{\mathrm{Ni}}$ we assumed that the extinction along the SN line of sight is constant for $\Delta t \leq 320 \mathrm{~d}$. However, normal SNe II are dust factories ${ }^{14}$, where the onset of the dust formation is different for each SN. In some cases, the dust formation begins as early as $\Delta t \sim 100-200 \mathrm{~d}$ (e.g. SN 2007od, Andrews et al. 2010, Inserra et al. 2011; SN 2011ja, Andrews et al. 2016; SN 2017eaw, Rho et al. 2018, Tinyanont et al. 2019). The latter indicates that some normal SNe II may experience a non-negligible increase of the extinction at $\Delta t \leq 320 \mathrm{~d}$, with a consequent decrease in their ${ }^{56} \mathrm{Ni}$ masses inferred from optical light.

In the case of SN 2007od, the newly formed dust decreases the $\log M_{56 \mathrm{Ni}}$ inferred from optical light in $\sim 0.8 \mathrm{dex}$ (see Section 4.3.2). On the other hand, for SN 2017eaw (which shows evidence of dust formation at $\Delta t \sim 120 \mathrm{~d}$, Rho et al. 2018) we measure a $\log M_{56 \mathrm{Ni}}$ of $-1.087 \mathrm{dex}$ $\left(M_{56 \mathrm{Ni}}=0.083 \mathrm{M}_{\odot}\right)$. This value is about $0.1-0.2$ dex greater than the predicted from the NMS relation. Moreover, our $M_{56} \mathrm{Ni}$ estimate is consistent with the ${ }^{56} \mathrm{Ni}$ mass of $0.084 \mathrm{M}_{\odot}$ used in the models found by Rho et al. (2018) to be consistent with the near-IR spectra of SN 2017eaw. Therefore, the early dust formation does not necessarily translate into a non-negligible increase of the extinction.

In the case of strong extinction due to newly formed dust, if it only affects to $\mathrm{SNe} 2007 \mathrm{od}$, and possibly to SN 2010aj in our set, then the fraction of these events is around 1-2 per cent. Therefore, they should not be a severe contaminant in the mean ${ }^{56} \mathrm{Ni}$ mass of normal SNe II.

\subsection{Future improvements}

Future works on improving the precision of $\left\langle M_{56 \mathrm{Ni}}\right\rangle$ should focus on reducing the random and $\mathrm{ZP}^{\mathrm{BC}}$ errors.

The $\mathrm{ZP}_{x}^{\mathrm{BC}}$ values we present in Section 4.2 are based on only $15 \mathrm{SNe}$, so their errors could be misestimated due to the small sample size. Indeed, the real error on $\mathrm{ZP}_{x}^{\mathrm{BC}}$ for the $I$-band (the preferred one to measure $\log M_{56} \mathrm{Ni}$ ) can be as low as $0.07 \mathrm{mag}$ or as large as $0.19 \mathrm{mag}$ at a confidence level of 99 per cent ${ }^{15}$. Moreover, the small sample size of our

14 The amount of newly formed dust, however, is still unclear (e.g. Priestley et al. 2020).

15 Assuming that $\mathrm{ZP}_{I}^{\mathrm{BC}}$ has a normal parent distribution with standard deviation $\sigma$, for which the quantity $(\hat{\sigma} / \sigma)^{2} \nu$ has a chisquare distribution with $\nu$ degrees of freedom (e.g. Lu 1960). 
$\mathrm{BC}$ calibration set does not allow us to robustly detect outliers. For example, using the Chauvenet's criterion, we find that $\mathrm{SN} 2014 \mathrm{G}$ is a possible outlier in the $\mathrm{ZP}_{I}^{\mathrm{BC}}$ distribution (Fig. 10). On the other hand, using the Chauvenet's criterion over $10^{4}$ bootstrap resampling, we find that SN 2014G is consistent with the $\mathrm{ZP}_{I}^{\mathrm{BC}}$ distribution in 65 per cent of the realizations. Increasing the number of $\mathrm{SNe}$ used to calibrate BCs (i.e. observed at optical and near-IR filters in the radioactive tail) is, therefore, a necessary step to improve the $\mathrm{ZP}^{\mathrm{BC}}$ error estimation.

One of the current surveys providing optical photometry is the ZTF, which observe about 2300 normal SNe II brighter than 20 mag per year (Feindt et al. 2019). Based on our SN sample, the $R$-band magnitude of normal SNe II during the first $50 \mathrm{~d}$ of the radioactive tail is between $1.5-3.8 \mathrm{mag}$ dimmer than at the maximum light, with an average of 2.5 mag. This means that roughly 3 per cent of all normal SNe II observed by the ZTF have $r_{\text {ZTF }}$ photometry useful to estimate $\log M_{56 \mathrm{Ni}}$ with the radioactive tail photometry. Therefore, it is possible to construct an SN set of similar size to that we used here with two years of ZTF data. Within a few years, the Rubin Observatory Legacy Survey of Space and Time (LSST) will be the main source of photometric data, which will observe $\sim 10^{5}$ SNe II per year (Lien \& Fields 2009). With one year of LSST data ( 2500 normal SNe II with radioactive tail photometry), it will be feasible to reduce the random error in $\left\langle M_{56_{\mathrm{Ni}}}\right\rangle$ from 9 per cent (estimated in this work) to around 2 per cent.

The NMS relation provides a method to measure $\log M_{56} \mathrm{Ni}$ virtually independent on the radioactive tail photometry. Therefore, the $\log M_{56}{ }_{\mathrm{Ni}}$ estimates computed with the radioactive tail luminosity and the NMS relation could be combined to further reduce the random error. Since the transition phase lasts $\lesssim 30 \mathrm{~d}, \log M_{56} \mathrm{Ni}$ measurements with the NMS relation require light curves sampled with a cadence of $\sim 5 \mathrm{~d}$. As we see in Figure 20, the cadence of the ZTF (around $3 \mathrm{~d}$ ) is more than enough to estimate the steepness parameter. In the case of the LSST, in order to have light curves sampled with a cadence of $\sim 5 \mathrm{~d}$, it will be necessary to combine light curves in different bands into a single one.

Finally, based on the empirical correlation between absolute magnitude and expansion velocity at $\Delta t=50 \mathrm{~d}\left(v_{50 \mathrm{~d}}\right)$ (e.g. Hamuy \& Pinto 2002; Hamuy 2003), we expect a relation between $\log M_{56_{\mathrm{Ni}}}, v_{50 \mathrm{~d}}$, and $S_{x}$. If confirmed, the latter relation will provide a method to measure $\log M_{56} \mathrm{Ni}$ independent of the distance and colour excess.

\section{SUMMARY AND CONCLUSIONS}

In this work we computed the ${ }^{56} \mathrm{Ni}$ masses of 110 normal $\mathrm{SNe}$ II from their luminosities in the radioactive tail. To estimate those luminosities we employed the BC technique. We used $15 \mathrm{SNe}$ with $B V(r) R(i) I J H K$ photometry and three theoretical spectral models to calibrate the $\mathrm{BC}$ values. In order to convert ${ }^{56} \mathrm{Ni}$ masses to iron masses, we used iron isotope ratios of $\mathrm{CC}$ nucleosynthesis models. We also analysed the correlation of the ${ }^{56} \mathrm{Ni}$ mass on the steepness parameter and on the absolute magnitude at $\Delta t=50 \mathrm{~d}$.

Our main conclusion are the following:

(1) The $I$ - and $i$-band are best suited to estimate radioac- tive tail luminosities through the $\mathrm{BC}$ technique. In particular, the $\mathrm{BC}_{V}$ value is not constant as reported in previous studies but it is correlated with the $V-I$ colour.

(2) We obtained $\left\langle M_{56} 6_{\mathrm{Ni}}\right\rangle=0.037 \pm 0.005 \mathrm{M}_{\odot}$ for normal SNe II, which translates into a $\bar{y}_{\mathrm{Fe}}$ of $0.040 \pm 0.005 \mathrm{M}_{\odot}$. Combining this result with recent mean ${ }^{56} \mathrm{Ni}$ mass measurements for other CC SN subtypes, we estimated a mean CC SN iron yield $<0.068 \mathrm{M}_{\odot}$. The contribution of normal SNe II to this yield is $>36$ per cent.

(3) The relation between $\log M_{56 \mathrm{Ni}}$ and $S_{V}$ suggested by Elmhamdi et al. (2003b) is poorly suited to estimate $M_{56} \mathrm{Ni}$. Instead we proposed the NMS method, based on the correlation of $\log M_{56 \mathrm{Ni}}$ on $M_{x}^{50 \mathrm{~d}}$ and $\log S_{x}$, which allows to measure $\log M_{56 \mathrm{Ni}}$ with a precision of 0.13 dex. Using the $r_{\text {ZTF }}$ photometry of 28 normal SNe II from the ZTF BTS, we obtained further evidence supporting the usefulness of the NMS relation to measure $\log M_{56} \mathrm{Ni}$.

Future works with ZTF and LSST data during the first years of operation will allow to verify our $\left\langle M_{56 \mathrm{Ni}}\right\rangle$ measurement. In particular, it will be feasible to reduce its random error by a factor of four with one year of LSST data. On the other hand, to reduce the error due to the BC ZP uncertainty, it will be necessary to carry out an observational campaign to increase the number of normal SNe II observed with optical and near-IR filters during the radioactive tail.

\section{ACKNOWLEDGEMENTS}

This paper is part of a project that has received funding from the European Research Council (ERC) under the European Union's Seventh Framework Programme, Grant agreement No. 833031 (PI Dan Maoz). MR thanks the support of the National Agency for Research and Development, ANIDPFCHA/Doctorado-Nacional/2020-21202606. This research has made use of the NASA/IPAC Extragalactic Database (NED) which is operated by the Jet Propulsion Laboratory, California Institute of Technology, under contract with the National Aeronautics and Space Administration. This work has made use of the Weizmann Interactive Supernova Data Repository (https://www.wiserep.org).

\section{DATA AVAILABILITY}

The data underlying this article will be shared on reasonable request to the corresponding author.

\section{REFERENCES}

Afsariardchi N., et al., 2019, ApJ, 881, 22

Afsariardchi N., Drout M. R., Khatami D., Matzner C. D., Moon D.-S., Ni Y. Q., 2020, arXiv e-prints, p. arXiv:2009.06683

Anand G. S., Rizzi L., Tully R. B., 2018, AJ, 156, 105

Anderson J. P., 2019, A\&A, 628, A7

Anderson J. P., et al., 2014, ApJ, 786, 67

Andrews J. E., et al., 2010, ApJ, 715, 541

Andrews J. E., et al., 2011, ApJ, 731, 47

Andrews J. E., et al., 2016, MNRAS, 457, 3241

Andrews J. E., et al., 2019, ApJ, 885, 43

Angus J. E., 1994, Journal of the Royal Statistical Society. Series D (The Statistician), 43, 395 
Arcavi I., et al., 2012, ApJ, 756, L30

Arcavi I., et al., 2017, Nature, 551, 210

Arnett W. D., 1982, ApJ, 253, 785

Barbarino C., et al., 2015, MNRAS, 448, 2312

Barbon R., Ciatti F., Rosino L., 1982, A\&A, 116, 35

Bayless A. J., et al., 2013, ApJ, 764, L13

Bellm E. C., et al., 2019, PASP, 131, 018002

Benetti S., Cappellaro E., Turatto M., 1991, A\&A, 247, 410

Benetti S., Cappellaro E., Turatto M., della Valle M., Mazzali P. A., Gouiffes C., 1994, A\&A, 285, 147

Benetti S., et al., 2001, MNRAS, 322, 361

Bersten M. C., Hamuy M., 2009, ApJ, 701, 200

Bersten M. C., Benvenuto O., Hamuy M., 2011, ApJ, 729, 61

Bessell M., Murphy S., 2012, PASP, 124, 140

Blanc G., Greggio L., 2008, New Astron., 13, 606

Blanton E. L., Schmidt B. P., Kirshner R. P., Ford C. H., Chromey F. R., Herbst W., 1995, AJ, 110, 2868

Bohlin R. C., Gilliland R. L., 2004, AJ, 127, 3508

Bose S., et al., 2013, MNRAS, 433, 1871

Bose S., et al., 2015a, MNRAS, 450, 2373

Bose S., et al., 2015b, ApJ, 806, 160

Bose S., Kumar B., Misra K., Matsumoto K., Kumar B., Singh M., Fukushima D., Kawabata M., 2016, MNRAS, 455, 2712

Bose S., et al., 2018, ApJ, 862, 107

Bose S., et al., 2019, ApJ, 873, L3

Bostroem K. A., et al., 2019, MNRAS, 485, 5120

Bostroem K. A., et al., 2020, ApJ, 895, 31

Brown P. J., et al., 2009, AJ, 137, 4517

Bullivant C., et al., 2018, MNRAS, 476, 1497

Burnham K. P., Anderson D. R., 2002, Model Selection and Multimodel Inference, 2nd edn. Springer-Verlag, New York

Burrows A., Vartanyan D., 2021, Nature, 589, 29

Buta R. J., 1982, PASP, 94, 578

Buta R. J., Keel W. C., 2019, MNRAS, 487, 832

Cappellaro E., Danziger I. J., della Valle M., Gouiffes C., Turatto M., 1995, A\&A, 293, 723

Carlin J. L., et al., 2016, ApJ, 828, L5

Chauvenet W., 1863, A manual of spherical and practical astronomy

Childress M. J., et al., 2016, Publ. Astron. Soc. Australia, $33, \mathrm{e} 055$

Cleveland W. S., Grosse E., Shyu W. M., 1992, in Chambers J. M., Hastie T. J., eds, , Statistical models in S. Chapman and Hall, London, Chapt. 8, pp 309-376

Clocchiatti A., et al., 1996, AJ, 111, 1286

Cohen M., Walker R. G., Carter B., Hammersley P., Kidger M., Noguchi K., 1999, AJ, 117, 1864

Cohen M., Wheaton W. A., Megeath S. T., 2003, AJ, 126, 1090

Curtis S., Ebinger K., Fröhlich C., Hempel M., Perego A., Liebendörfer M., Thielemann F.-K., 2019, ApJ, 870, 2

Dall'Ora M., et al., 2014, ApJ, 787, 139

Dastidar R., et al., 2018, MNRAS, 479, 2421

Dastidar R., et al., 2019, MNRAS, 490, 1605

Dastidar R., et al., 2021, MNRAS,

Dessart L., Hillier D. J., 2011, MNRAS, 410, 1739

Dessart L., et al., 2008, ApJ, 675, 644

Dessart L., Hillier D. J., Waldman R., Livne E., 2013, MNRAS, 433,1745

Dessart L., Hillier D. J., Woosley S., Livne E., Waldman R., Yoon S.-C., Langer N., 2015, MNRAS, 453, 2189

Dessart L., Hillier D. J., Woosley S., Livne E., Waldman R., Yoon S.-C., Langer N., 2016, MNRAS, 458, 1618

Dhungana G., et al., 2016, ApJ, 822, 6

Dimai A., 2016, Transient Name Server Discovery Report, 2016-673, 1

Dong Y., et al., 2020, ApJ, 906, 56

Elias-Rosa N., et al., 2011, ApJ, 742, 6

Elmhamdi A., et al., 2003a, MNRAS, 338, 939
Elmhamdi A., Chugai N. N., Danziger I. J., 2003b, A\&A, 404, 1077

Faran T., et al., 2014a, MNRAS, 442, 844

Faran T., et al., 2014b, MNRAS, 445, 554

Feindt U., Nordin J., Rigault M., Brinnel V., Dhawan S., Goobar A., Kowalski M., 2019, J. Cosmology Astropart. Phys., 2019, 005

Fitzpatrick E. L., 1999, PASP, 111, 63

Förster F., et al., 2018, Nature Astronomy, 2, 808

Förster F., et al., 2021, AJ, 161, 242

Foster C., et al., 2014, MNRAS, 442, 3544

Fraser M., et al., 2011, MNRAS, 417, 1417

Freedman W. L., et al., 2019, ApJ, 882, 34

Freedman W. L., et al., 2020, ApJ, 891, 57

Fremling C., et al., 2020, ApJ, 895, 32

Fukugita M., Ichikawa T., Gunn J. E., Doi M., Shimasaku K., Schneider D. P., 1996, AJ, 111, 1748

Gal-Yam A., 2017, Observational and Physical Classification of Supernovae. p. 195, doi:10.1007/978-3-319-21846-5_35

Galbany L., et al., 2016, AJ, 151, 33

Graham M. J., et al., 2019, PASP, 131, 078001

Graziani R., Courtois H. M., Lavaux G., Hoffman Y., Tully R. B., Copin Y., Pomarède D., 2019, MNRAS, 488, 5438

Gurugubelli U. K., Sahu D. K., Anupama G. C., Chakradhari N. K., 2008, Bulletin of the Astronomical Society of India, 36,79

Gutiérrez C. P., et al., 2017a, ApJ, 850, 89

Gutiérrez C. P., et al., 2017b, ApJ, 850, 90

Gutiérrez C. P., et al., 2020, MNRAS, 496, 95

Hamuy M. A., 2001, PhD thesis, The University of Arizona

Hamuy M., 2003, ApJ, 582, 905

Hamuy M., Pinto P. A., 2002, ApJ, 566, L63

Hamuy M., Suntzeff N. B., Gonzalez R., Martin G., 1988, AJ, 95,63

Harutyunyan A. H., et al., 2008, A\&A, 488, 383

Hayes D. S., Latham D. W., 1975, ApJ, 197, 593

Hendry M. A., et al., 2005, MNRAS, 359, 906

Hendry M. A., et al., 2006, MNRAS, 369, 1303

Hicken M., et al., 2017, ApJS, 233, 6

Hoeflich P., 2017, Explosion Physics of Thermonuclear Supernovae and Their Signatures. p. 1151, doi:10.1007/978-3-319-21846-5_56

Hosseinzadeh G., et al., 2018, ApJ, 861, 63

Huang F., et al., 2015, ApJ, 807, 59

Huang F., et al., 2016, ApJ, 832, 139

Huang F., et al., 2018, MNRAS, 475, 3959

Inserra C., et al., 2011, MNRAS, 417, 261

Inserra C., et al., 2012, MNRAS, 422, 1122

Inserra C., et al., 2013, A\&A, 555, A142

Itagaki K., 2016, Transient Name Server Discovery Report, 2016-234, 1

Iwamoto K., Brachwitz F., Nomoto K., Kishimoto N., Umeda H., Hix W. R., Thielemann F.-K., 1999, ApJS, 125, 439

Jang I. S., Lee M. G., 2017, ApJ, 836, 74

Jeffery D. J., 1999, arXiv e-prints, pp astro-ph/9907015

Jerkstrand A., Fransson C., Kozma C., 2011, A\&A, 530, A45

Jerkstrand A., Smartt S. J., Fraser M., Fransson C., Sollerman J., Taddia F., Kotak R., 2014, MNRAS, 439, 3694

Kobayashi C., Umeda H., Nomoto K., Tominaga N., Ohkubo T., 2006, ApJ, 653, 1145

Kourkchi E., Courtois H. M., Graziani R., Hoffman Y., Pomarède D., Shaya E. J., Tully R. B., 2020, AJ, 159, 67

Kozyreva A., Nakar E., Waldman R., 2019, MNRAS, 483, 1211

Krisciunas K., et al., 2009, AJ, 137, 34

Leonard D. C., et al., 2002, AJ, 124, 2490

Li W., Wang X., Van Dyk S. D., Cuilland re J.-C., Foley R. J., Filippenko A. V., 2007, ApJ, 661, 1013

Li W., et al., 2011, MNRAS, 412, 1441 
Liao J., Sun G., Gao X., 2019, Transient Name Server Discovery Report, 2019-1124, 1

Liddle A. R., 2007, MNRAS, 377, L74

Lien A., Fields B. D., 2009, J. Cosmology Astropart. Phys., 2009, 047

Lisakov S. M., Dessart L., Hillier D. J., Waldman R., Livne E., 2017, MNRAS, 466, 34

Lu J. Y., 1960, Journal of Farm Economics, 42, 910

Lusk J. A., Baron E., 2017, PASP, 129, 044202

Maguire K., et al., 2010, MNRAS, 404, 981

Maoz D., Graur O., 2017, ApJ, 848, 25

McQuinn K. B. W., Skillman E. D., Dolphin A. E., Berg D., Kennicutt R., 2017, AJ, 154, 51

Meikle W. P. S., et al., 2011, ApJ, 732, 109

Meza N., Anderson J. P., 2020, A\&A, 641, A177

Minkowski R., 1941, PASP, 53, 224

Misra K., Pooley D., Chandra P., Bhattacharya D., Ray A. K., Sagar R., Lewin W. H. G., 2007, MNRAS, 381, 280

Monachesi A., Bell E. F., Radburn-Smith D. J., Bailin J., de Jong R. S., Holwerda B., Streich D., Silverstein G., 2016, MNRAS, 457, 1419

Morozova V., Piro A. L., Valenti S., 2018, ApJ, 858, 15

Müller-Bravo T. E., et al., 2020, MNRAS, 497, 361

Müller T., Prieto J. L., Pejcha O., Clocchiatti A., 2017, ApJ, 841,127

Munari U., Henden A., Belligoli R., Castellani F., Cherini G., Righetti G. L., Vagnozzi A., 2013, New Astron., 20, 30

Nakaoka T., et al., 2018, ApJ, 859, 78

Nomoto K., Hashimoto M., Tsujimoto T., Thielemann F. K., Kishimoto N., Kubo Y., Nakasato N., 1997, Nuclear Phys. A, 616, 79

Olivares E. F., et al., 2010, ApJ, 715, 833

Pastorello A., et al., 2004, MNRAS, 347, 74

Pastorello A., et al., 2006, MNRAS, 370, 1752

Pastorello A., et al., 2007, MNRAS, 376, 1301

Pastorello A., et al., 2009a, MNRAS, 394, 2266

Pastorello A., et al., 2009b, A\&A, 500, 1013

Pejcha O., Prieto J. L., 2015a, ApJ, 799, 215

Pejcha O., Prieto J. L., 2015b, ApJ, 806, 225

Pennypacker C. R., et al., 1989, AJ, 97, 186

Perley D. A., et al., 2020, ApJ, 904, 35

Phillips M. M., et al., 2013, ApJ, 779, 38

Pignata G., 2013, in Massive Stars: From alpha to Omega. p. 176

Poznanski D., Prochaska J. X., Bloom J. S., 2012, MNRAS, 426,1465

Pozzo M., et al., 2006, MNRAS, 368, 1169

Priestley F. D., Bevan A., Barlow M. J., De Looze I., 2020, MNRAS, 497, 2227

Pumo M. L., Zampieri L., 2011, ApJ, 741, 41

Pumo M. L., Zampieri L., 2013, MNRAS, 434, 3445

Quimby R. M., Wheeler J. C., Höflich P., Akerlof C. W., Brown P. J., Rykoff E. S., 2007, ApJ, 666, 1093

Reguitti A., et al., 2021, MNRAS, 501, 1059

Rho J., Geballe T. R., Banerjee D. P. K., Dessart L., Evans A., Joshi V., 2018, ApJ, 864, L20

Richmond M. W., 2014, Journal of the American Association of Variable Star Observers (JAAVSO), 42, 333

Riess A. G., et al., 2016, ApJ, 826, 56

Riess A. G., Casertano S., Yuan W., Macri L. M., Scolnic D., 2019, ApJ, 876, 85

Rizzi L., Tully R. B., Makarov D., Makarova L., Dolphin A. E., Sakai S., Shaya E. J., 2007, ApJ, 661, 815

Rodríguez Ó., Clocchiatti A., Hamuy M., 2014, AJ, 148, 107

Rodríguez Ó., et al., 2019, MNRAS, 483, 5459

Rodríguez Ó., et al., 2020, MNRAS, 494, 5882

Roy R., et al., 2011a, MNRAS, 414, 167

Roy R., et al., 2011b, ApJ, 736, 76

Rubenstein E. P., 1999, IAU Circ., 7319, 2
Rubin A., et al., 2016, ApJ, 820, 33

Ruiz-Lapuente P., Kidger M., Lopez R., Canal R., 1990, AJ, 100, 782

Saha A., Thim F., Tammann G. A., Reindl B., Sand age A., 2006, ApJS, 165, 108

Sahu D. K., Anupama G. C., Srividya S., Muneer S., 2006, MNRAS, 372, 1315

Sanders N. E., et al., 2015, ApJ, 799, 208

Schlafly E. F., Finkbeiner D. P., 2011, ApJ, 737, 103

Schlegel E. M., 1990, MNRAS, 244, 269

Schlegel D. J., Finkbeiner D. P., Davis M., 1998, ApJ, 500, 525

Schmidt B. P., et al., 1993, AJ, 105, 2236

Scholz F. W., Stephens M. A., 1987, Journal of the American Statistical Association, 82, 918

Schwarz G., 1978, Annals of Statistics, 6, 461

Sharon A., Kushnir D., 2020, MNRAS, 496, 4517

Shaya E. J., Tully R. B., Hoffman Y., Pomarède D., 2017, ApJ, 850,207

Shivvers I., et al., 2017, PASP, 129, 054201

Silverman J. M., et al., 2017, MNRAS, 467, 369

Singh A., Srivastav S., Kumar B., Anupama G. C., Sahu D. K., 2018, MNRAS, 480, 2475

Singh A., Kumar B., Moriya T. J., Anupama G. C., Sahu D. K., Brown P. J., Andrews J. E., Smith N., 2019, ApJ, 882, 68

Smartt S. J., 2009, ARA\&A, 47, 63

Smartt S. J., 2015, Publ. Astron. Soc. Australia, 32, e016

Spiro S., et al., 2014, MNRAS, 439, 2873

Stritzinger M., Suntzeff N. B., Hamuy M., Challis P., Demarco R., Germany L., Soderberg A. M., 2005, PASP, 117, 810

Sukhbold T., Ertl T., Woosley S. E., Brown J. M., Janka H. T., 2016, ApJ, 821, 38

Suwa Y., Tominaga N., Maeda K., 2019, MNRAS, 483, 3607

Szalai T., et al., 2019, ApJ, 876, 19

Taddia F., et al., 2016, A\&A, 588, A5

Takáts K., et al., 2014, MNRAS, 438, 368

Takáts K., et al., 2015, MNRAS, 450, 3137

Tartaglia L., et al., 2018, ApJ, 853, 62

Tartaglia L., et al., 2021, ApJ, 907, 52

Terreran G., et al., 2016, MNRAS, 462, 137

Terreran G., et al., 2017, Nature Astronomy, 1, 713

Thompson L. A., 1982, ApJ, 257, L63

Tikhonov N. A., Galazutdinova O. A., 2020, Astrophysical Bulletin, 75, 384

Tinyanont S., et al., 2019, ApJ, 873, 127

Tomasella L., et al., 2013, MNRAS, 434, 1636

Tomasella L., et al., 2018, MNRAS, 475, 1937

Tsvetkov D. Y., 1983, Peremennye Zvezdy, 22, 39

Tsvetkov D. Y., 2014, Peremennye Zvezdy, 34, 2

Tsvetkov D. Y., Volnova A. A., Shulga A. P., Korotkiy S. A., Elmhamdi A., Danziger I. J., Ereshko M. V., 2006, A\&A, 460,769

Tsvetkov D. Y., Muminov M., Burkhanov O., Kahharov B., 2007, Peremennye Zvezdy, 27, 5

Tsvetkov D. Y., et al., 2018, Astronomy Letters, 44, 315

Tsvetkov D. Y., et al., 2019, MNRAS, 487, 3001

Tully R. B., Rizzi L., Shaya E. J., Courtois H. M., Makarov D. I., Jacobs B. A., 2009, AJ, 138, 323

Turatto M., Cappellaro E., Benetti S., Danziger I. J., 1993, MNRAS, 265, 471

Turatto M., et al., 1998, ApJ, 498, L129

Ugliano M., Janka H.-T., Marek A., Arcones A., 2012, ApJ, 757,69

Valenti S., et al., 2015, MNRAS, 448, 2608

Valenti S., et al., 2016, MNRAS, 459, 3939

Van Dyk S. D., 2013, AJ, 146, 24

Van Dyk S. D., et al., 2012, AJ, 143, 19

Van Dyk S. D., et al., 2019, ApJ, 875, 136

Vinkó J., et al., 2006, MNRAS, 369, 1780 
Vinkó J., et al., 2009, ApJ, 695, 619

Weaver T. A., Woosley S. E., 1980, in Ninth Texas Symposium on Relativistic Astrophysics. pp 335-357, doi:10.1111/j.1749-6632.1980.tb15942.x

Wiggins P., 2017, Transient Name Server Discovery Report, 2017-548, 1

Woosley S. E., Weaver T. A., 1995, ApJS, 101, 181

Wyrzykowski Ł., et al., 2014, Acta Astron., 64, 197

Yaron O., et al., 2017, Nature Physics, 13, 510

Young T. R., 2004, ApJ, 617, 1233

Yuan F., et al., 2016, MNRAS, 461, 2003

Yuan W., Riess A. G., Macri L. M., Casertano S., Scolnic D. M., 2019, ApJ, 886, 61

Zgirski B., et al., 2017, ApJ, 847, 88

Zhang T., Wang X., Li W., Zhou X., Ma J., Jiang Z., Chen J., 2006, AJ, 131, 2245

Zhang J., et al., 2014, ApJ, 797, 5

de Jaeger T., et al., 2019, MNRAS, 490, 2799

de Jaeger T., Stahl B. E., Zheng W., Filippenko A. V., Riess A. G., Galbany L., 2020, MNRAS, 496, 3402

\section{APPENDIX A: SYNTHETIC MAGNITUDES}

Given a SED $f_{\lambda}$ (in $\operatorname{erg~s}^{-1} \mathrm{~cm}^{-2} \AA^{-1}$ ), we can compute the synthetic magnitude in the $x$-band using

$m_{x}=-2.5 \log \int d \lambda S_{x, \lambda} \frac{\lambda f_{\lambda}}{h c}+\mathrm{ZP}_{x}^{\mathrm{mag}}$

(e.g. Hamuy 2001). Here $\lambda$ is the observed wavelength (in $\AA$ ),$S_{x, \lambda}$ is the peak-normalized $x$-band transmission function (considering a photon-counting detector), $h c=1.986 \times 10^{-8} \operatorname{erg} \AA$, and $\mathrm{ZP}_{x}^{\operatorname{mag}}$ is the zero-point for the magnitude system. To compute $\mathrm{ZP}_{x}^{\mathrm{mag}}$ values in the Vega system, we use equation (A1) along with the Vega SED published by Bohlin \& Gilliland $(2004)^{16}$ and the Vega magnitudes published by Fukugita et al. (1996): $B=0.03$, $V=0.03$, and $I=0.024 \mathrm{mag}$, and by Cohen et al. (1999): $J=-0.001, H=0.000$, and $K=-0.001 \mathrm{mag}$. For JohnsonKron-Cousins BVRI bands we adopt the transmission functions given in Stritzinger et al. (2005), while for 2MASS JHK bands we adopt the transmission of Cohen et al. $(2003)^{17}$. To compute $\mathrm{ZP}_{x}^{\mathrm{mag}}$ values in the $\mathrm{AB}$ system, we use equation (A1), $f_{\lambda}=1 / \lambda^{2}$, and $m_{x}=-2.408$. For the Sloan gri bands we use the transmission functions of the SDSS Data Release $7^{18}$. To compute the $r_{\mathrm{ZTF}}$ transmission function, we use equation (1) of Pastorello et al. (2007), adopting the corresponding ZTF filter transmission ${ }^{17}$ and CCD quantum efficiency ${ }^{19}$, the atmospheric extinction at Palomar Observatory of Hayes \& Latham (1975) (assuming an airmass of 1.2) combined with atmospheric telluric lines, a throughput. Column 3 of Table A1 lists the $\mathrm{ZP}_{x}^{\mathrm{mag}}$ values.
Wygoda N., Elbaz Y., Katz B., 2019, MNRAS, 484, 3941 standard aluminium reflectivity curve, and a constant lens

Table A1. Properties of the filters used in this work.

\begin{tabular}{lcccccc}
\hline$x$ & System & $\begin{array}{c}\mathrm{ZP}_{x}^{\text {mag }} \\
(\mathrm{mag})\end{array}$ & $\begin{array}{c}\mathrm{ZP}_{x}^{\text {mflux }} \\
(\mathrm{mag})\end{array}$ & $\begin{array}{c}\bar{\lambda}_{x} \\
(\AA)\end{array}$ & $R_{\bar{\lambda}_{x}}$ & $R_{x}^{\mathrm{pt}}$ \\
\hline$B$ & Vega & 15.300 & -20.462 & 4610 & 3.86 & 3.99 \\
$g$ & $\mathrm{AB}$ & 15.329 & -20.770 & 4860 & 3.62 & 3.68 \\
$V$ & Vega & 14.865 & -21.074 & 5600 & 2.96 & 3.04 \\
$r$ & $\mathrm{AB}$ & 14.986 & -21.361 & 6330 & 2.49 & 2.55 \\
$r_{\text {ZTF }}$ & $\mathrm{AB}$ & 15.212 & -21.436 & 6490 & 2.40 & 2.45 \\
$R$ & Vega & 15.053 & -21.629 & 6610 & 2.33 & 2.40 \\
$i$ & $\mathrm{AB}$ & 14.710 & -21.780 & 7450 & 1.93 & 1.91 \\
$I$ & Vega & 14.538 & -22.354 & 8090 & 1.68 & 1.71 \\
$J$ & Vega & 13.729 & -23.787 & 12470 & 0.80 & 0.80 \\
$H$ & Vega & 13.412 & -24.886 & 16520 & 0.51 & 0.51 \\
$K$ & Vega & 12.691 & -25.948 & 21630 & 0.35 & 0.35 \\
\hline
\end{tabular}

\section{APPENDIX B: PHOTOMETRIC SED}

To compute pSEDs, we need to convert $x$-band magnitudes to monochromatic fluxes $\bar{f}_{x}$ (in $\operatorname{erg~s}^{-1} \mathrm{~cm}^{-2} \AA^{-1}$ ). For this, we use $f_{\lambda}=\bar{f}_{x}$ in equation (A1), thus obtaining

$\bar{f}_{x}=10^{-0.4\left(m_{x}-\mathrm{ZP}_{x}^{\mathrm{mflux}}\right)}$,

where

$\mathrm{ZP}_{x}^{\text {mflux }}=2.5 \log \int d \lambda S_{x, \lambda} \frac{\lambda}{h c}+\mathrm{ZP}_{x}^{\mathrm{mag}}$.

Values of $\mathrm{ZP}_{x}^{\mathrm{mflux}}$ are reported in Column 4 of Table A1.

The effective wavelength of the $x$-band as a function of the SED is given by

$\lambda_{x}=\frac{\int d \lambda S_{x, \lambda} \lambda^{2} f_{\lambda}}{\int d \lambda S_{x, \lambda} \lambda f_{\lambda}}$

(e.g. Bessell \& Murphy 2012). To estimate this value for the $B V R I J H K$ bands, we use the theoretical spectral models of D13, J14, and L17 during the radioactive tail, adopting the median as the representative effective wavelength $\bar{\lambda}_{x}$. These values are summarized in Column 5 of Table A1. In Column 6 we report the total-to-selective extinction ratio for $\bar{\lambda}_{x}\left(R_{\bar{\lambda}_{x}}\right)$, assuming the extinction curve of Fitzpatrick (1999) with $R_{V}=3.1$. We also compute the $R_{\bar{\lambda}_{x}}$ values for the photospheric and transition phase $\left(R_{x}^{\mathrm{pt}}\right)$. Those values are listed in Column 7 of Table A1.

To obtain monochromatic fluxes corrected for reddening $\left(\bar{f}_{x}^{\text {corr }}\right)$ we use

$\bar{f}_{x}^{\mathrm{corr}}=\bar{f}_{x} \cdot 10^{0.4\left(E_{B-V}^{\mathrm{G}}+E_{B-V}^{\mathrm{h}}\right) R_{\bar{\lambda}_{x}} .}$

Using the set of $\left(\bar{\lambda}_{x}, \bar{f}_{x}^{\text {corr }}\right)$ values, we construct the pSED, from which the quasi-bolometric flux is computed with the trapezoidal rule, i.e.,

$F^{\text {BVRIJHK }}=\frac{1}{2} \sum_{i=1}^{6}\left(\bar{\lambda}_{x_{i+1}}-\bar{\lambda}_{x_{i}}\right)\left(\bar{f}_{x_{i+1}}^{\text {corr }}+\bar{f}_{x_{i}}^{\text {corr }}\right)$,

where $x_{j}=B, V, R, I, J, H, K$ for $j=1, \ldots, 7$. The error on $F^{B V R I J H K}$ due to error in photometry, $\sigma_{m_{x}}$, is given by

$\sigma_{F B V R I J H K}=\frac{\ln (10)}{5}\left(\left[\left(\bar{\lambda}_{x_{1}}-\bar{\lambda}_{x_{2}}\right) \bar{f}_{x_{1}}^{\text {corr }} \sigma_{m_{x_{1}}}\right]^{2}\right.$

16 https://ssb.stsci.edu/cdbs/current_calspec/alpha_lyr_stis_010.fits

17 Since they consider an energy-counting detector, we have to divide the transmissions by $\lambda$ before to use them in equation (A1).

18 http://classic.sdss.org/dr7/instruments/imager/index.html

19 https://github.com/ZwickyTransientFacility/ztf_information 
To estimate the error on $F^{B V R I J H K}$ due to uncertainties on $E_{B-V}^{\mathrm{G}}$ and $E_{B-V}^{\mathrm{h}}$, we define the pSED total-to-selective extinction ratio as

$R_{\mathrm{p}}=-\frac{2.5}{E_{B-V}^{\mathrm{G}}+E_{B-V}^{\mathrm{h}}} \log \left(\frac{F_{\mathrm{unc}}^{B V R I J H K}}{F^{B V R I J H K}}\right)$.

Here $F_{\text {unc }}^{B V R I J H}$ is the quasi-bolometric flux uncorrected for $E_{B-V}^{\mathrm{G}}$ and $E_{B-V}^{\mathrm{h}}$, i.e., replacing $\bar{f}_{x}^{\text {corr }}$ in equation (B5) by $\bar{f}_{x}$. With this, the error on $F^{B V R I J H K}$ due to the uncertainties on $E_{B-V}^{\mathrm{G}}$ and $E_{B-V}^{\mathrm{h}}$, in magnitude scale, is

$\sigma\left(2.5 \log \left(F^{\text {BVRIJHK }}\right)\right)=R_{\mathrm{p}} \sqrt{\sigma_{E_{B-V}^{\mathrm{G}}}^{2}+\sigma_{E_{B-V}^{\mathrm{h}}}^{2}}$.

\section{APPENDIX C: MODEL SELECTION}

Let a set of $N$ data $X_{i}=\left\{x_{i}, y_{i}, \sigma_{y_{i}}\right\}(i=1, \ldots, N)$ and a set of $M$ models $\left\{f_{j, \theta}\right\}(j=1, \ldots, M)$ with parameters $\theta$. To select the model that best represents the data, we use the Bayesian information criterion (BIC, Schwarz 1978), performing the following procedure. First, we compute the parameters $\theta$ of each model, maximizing its log-likelihood

$\ln \mathcal{L}\left(f_{j, \theta} \mid X_{i}\right)=-\frac{1}{2} \sum_{i}^{N}\left[\ln \left(\sigma_{i}^{2}\right)+\frac{\left(y_{i}-f_{j, \theta}\left(x_{i}\right)\right)^{2}}{\sigma_{i}^{2}}\right]$,

where $\sigma_{i}^{2}=\sigma_{y_{i}}^{2}+\sigma_{0}^{2}$, being $\sigma_{0}$ the error not accounted for the errors in $y_{i}$. Next, for each model we compute

$\mathrm{BIC}_{j}=-2 \ln \mathcal{L}_{j}^{\max }+k \ln N$

(Schwarz 1978), where $\ln \mathcal{L}_{j}^{\max }$ is the maximum loglikelihood and $k$ is the number of free parameters. Then, we evaluate the Bayesian weights

$p_{j}=e^{-0.5\left(\mathrm{BIC}_{j}-\mathrm{BIC}_{l}\right)}\left(\sum_{m=1}^{M} e^{-0.5\left(\mathrm{BIC}_{m}-\mathrm{BIC}_{l}\right)}\right)^{-1}$

(Burnham \& Anderson 2002), where $l$ is the index of the model with the lowest BIC value. As reference, if $p_{l} / p_{j}>13.0$ (e.g. Liddle 2007), then there is a strong evidence in favour of the $l$ th model over the $j$ th one. Finally, among all models with $p_{l} / p_{j} \leq 13.0$, by the principle of parsimony, we select the one with less parameters.

In the case of least-square regressions (e.g. when $\sigma_{y_{i}}$ values are not available), we replace equation (C2) by

$\mathrm{BIC}_{j}=\ln \left(s_{j}^{2}\right)+k / N \cdot \ln N$

(e.g. Rodríguez et al. 2019), where $s_{j}^{2}$ is the average of the squared residuals around the $j$ th model.

\section{APPENDIX D: ${ }^{56}$ NI MASS EQUATION}

Let a normal SN II with a set of $N$ measurements of $\left\{m_{x, i}\right\}$ magnitudes ( $K$-corrected, see Appendix I) at times $t_{i}$, and a set of parameters $\left\{t_{0}, \mu, E_{B-V}^{\mathrm{G}}, E_{B-V}^{\mathrm{h}}, z_{\text {helio }}^{\mathrm{SN}}\right\}$. The ${ }^{56} \mathrm{Ni}$ mass (in $\mathrm{M}_{\odot}$ ) can be computed using equations (17) and (18), which can be written as

$\log M_{56_{\mathrm{Ni}}}=\left\langle A_{i}-D_{i}\right\rangle+B$
Here the angle brackets denote the value that maximizes the log-likelihood of a constant-only model (equation $\mathrm{C} 1$ ) where, using $\mathrm{BC}_{x}=\mathrm{ZP}_{x}^{\mathrm{BC}}+\beta_{x} \Delta t / 100 \mathrm{~d}$,

$A_{i}=-\frac{m_{x, i}}{2.5}+\frac{\left(0.39-\beta_{x} / 2.5\right) t_{i}}{\left(1+z_{\text {helio }}^{\mathrm{SN}}\right) 100 \mathrm{~d}}$

$B=\frac{\mu-\mathrm{ZP}_{x}^{\mathrm{BC}}+A_{x}^{\mathrm{tot}}}{2.5}-\frac{\left(0.39-\beta_{x} / 2.5\right) t_{0}}{\left(1+z_{\text {helio }}^{\mathrm{SN}}\right) 100 \mathrm{~d}}-3.076$,

where $A_{x}^{\text {tot }}=\left(E_{B-V}^{\mathrm{G}}+E_{B-V}^{\mathrm{h}}\right) R_{\bar{\lambda}_{x}}$. To evaluate whether it is necessary to include the $D_{i}$ correction in equation (D1), we have to check the constancy of the $\log M_{56_{\mathrm{Ni}}}$ estimates with respect to $t_{i}$. For this, we use the model selection procedure given in Appendix $\mathrm{C}$ using the $\left\{t_{i}, A_{i}, \sigma_{A_{i}}\right\}$ data along with a zero-order and a linear polynomial. If data can be represented by a zero-order polynomial, then $D_{i}=0$ in equation (D1). In this case, the $\log M_{56} \mathrm{Ni}$ error is given by

$\sigma_{\log M_{56_{\mathrm{Ni}}}}=\sqrt{\sigma_{\left\langle A_{i}\right\rangle}^{2}+\sigma_{B}^{2}}$.

Here

$\sigma_{B}^{2}=\frac{\sigma_{\mu}^{2}+\sigma_{\mathrm{ZP}_{x}^{\mathrm{BC}}}^{2}+\sigma_{A_{x}^{\text {tot }}}^{2}}{6.25}+\left[\frac{\left(0.39-\beta_{x} / 2.5\right) \sigma_{t_{0}}}{\left(1+z_{\text {helio }}^{\mathrm{SN}}\right) 100 \mathrm{~d}}\right]^{2}$,

where $\sigma$ denotes the error in the subscripted quantity, and $\sigma_{\left\langle A_{i}\right\rangle}=\hat{\sigma}_{A, k} / \sqrt{N}$, being

$\hat{\sigma}_{X, k}=\left[\frac{1}{N-k} \sum_{i}^{N}\left(X_{i}-\left\langle X_{i}\right\rangle\right)^{2}\right]^{1 / 2}$

the sample standard deviation, and $k$ the number of free parameters.

If data are not well represented by a zero-order polynomial, then there is a dependence of the $\log M_{56_{\mathrm{Ni}}}$ estimates on $t_{i}$, so we have to include the $D_{i}$ term. If the time derivative of $A$ is negative as in the case of SN 2014G (lefthand side of Fig. 11), then we use the $D_{i}\left(T_{0}\right)$ term given by equations (19) and (20). The value of $T_{0}$ is computed through a log-likelihood maximization (equation $\mathrm{C} 1$ ). Once the value of $T_{0}$ is determined, we compute $\log M_{56} \mathrm{Ni}$ using equation (D1) and $D_{i}=D_{i}\left(T_{0}\right)$. The error in $\log M_{56 \mathrm{Ni}}$ is given by equation (D4) replacing $\sigma_{\left\langle A_{i}\right\rangle}$ by $\hat{\sigma}_{A-D, k} / \sqrt{N}$ if the $\log M_{56_{\mathrm{Ni}}}$ estimates are independent on $t_{i}$ (using the procedure given in Appendix C), otherwise we replace $\sigma_{\left\langle A_{i}\right\rangle}$ by $\hat{\sigma}_{A-D, k}$. If the time derivative of $A$ is positive as in the case of SN 2005cs (Fig. 12), then we assume the result obtained with $D_{i}=0$, and $\sigma_{\left\langle A_{i}\right\rangle}=\hat{\sigma}_{A, k}$ in equation (D4).

\section{APPENDIX E: LOG-NORMAL MEAN}

Let the quantity $x$ (e.g. $M_{56_{\mathrm{Ni}}}, D, S_{x}$ ) and its logtransformation $y=\log x$. If $y$ has a normal PDF with mean $\bar{y}$ and standard deviation $\sigma_{y}$, then $x$ has a log-normal distribution. The mean of the latter $\operatorname{PDF}(\bar{x})$ is not $10^{\bar{y}}$ (which indeed is the median) but

$\bar{x}=10^{\bar{y}+0.5 \ln (10) \sigma_{y}^{2}}$

(e.g. Angus 1994). The standard deviation of the $x$ PDF is

$\sigma_{x}=|\bar{x}| \sqrt{10^{\ln (10) \sigma_{y}^{2}}-1}$. 
On the other hand, if $x$ has a normal PDF then $\bar{y}$ is not $\log \bar{x}$ but

$\bar{y}=\log \bar{x}-0.5 \log \left(1+\left(\sigma_{x} / \bar{x}\right)^{2}\right)$,

while the standard deviation of the $y \mathrm{PDF}$ is

$\sigma_{y}=\sqrt{\ln \left(1+\left(\sigma_{x} / \bar{x}\right)^{2}\right)} / \ln (10)$.

\section{APPENDIX F: C3 METHOD}

The C3 method (Rodríguez et al. 2014, 2019) relies on the assumption that, during the plateau phase, all normal SNe II have similar linear $V-I$ versus $B-V$ C3s. For an SN with a measurement of $V-I$ and $B-V$ (corrected for $E_{B-V}^{\mathrm{G}}$ and $K$-correction, e.g. Rodríguez et al. 2019) at time $t_{i}$ (during the plateau phase), the host galaxy color excess is given by

$E_{B-V, i}^{\mathrm{h}}=A_{i}+\mathrm{ZP}_{\mathrm{C} 3}$.

Here $\mathrm{ZP}_{\mathrm{C} 3}$ is the zero-point for the $\mathrm{C} 3$ method, and

$A_{i}=\left[(V-I)_{i}-m_{\mathrm{C} 3} \cdot(B-V)_{i}\right] /\left(R_{V}-R_{I}-m_{\mathrm{C} 3}\right)$

(e.g. Rodríguez et al. 2019), where $R_{V}=3.1, R_{I}=1.72$, and $m_{\mathrm{C} 3}=0.45 \pm 0.07$ is the $V-I$ versus $B-V$ C3 slope (Rodríguez et al. 2019). For an $\mathrm{SN}$ with a set of $N$ measurement of $\left\{(B-V)_{i},(V-I)_{i}\right\}$, the $E_{B-V}^{\mathrm{h}}$ value is given by

$E_{B-V}^{\mathrm{h}}=\left\langle A_{i}\right\rangle+\mathrm{ZP}_{\mathrm{C} 3}$,

where the angle brackets denote the value that maximizes the log-likelihood of a constant-only model (equation C1).

The $\mathrm{ZP}_{\mathrm{C} 3}$ value can be computed from a set of reddening-free $\mathrm{SNe}$. For each of those $\mathrm{SNe}$, following equation (F3), we have $\mathrm{ZP}_{\mathrm{C} 3}^{\mathrm{SN}}=-\left\langle A_{i}\right\rangle^{\mathrm{SN}}$. Using this set of $\mathrm{ZP}_{\mathrm{C} 3}^{\mathrm{SN}}$ values, we calculate the mean and the $\hat{\sigma}$ value, which we adopt as $\mathrm{ZP}_{\mathrm{C} 3}$ and its error, respectively. To determine $\mathrm{ZP}_{\mathrm{C} 3}$ we use SNe 2005ay, 2008gz, 2013ej, and 2014cx, which are found to have low reddening by other methods (see Table L3), and SN 2003bn (Galbany et al. 2016), which is also affected by low reddening (e.g. Olivares E. et al. 2010). Using those $\mathrm{SNe}$, we obtain $\mathrm{ZP}_{\mathrm{C} 3}=-0.116 \pm 0.024 \mathrm{mag}$. This value is equivalent to the $\mathrm{ZP}_{\mathrm{C} 3}$ inferred by Rodríguez et al. (2019) from SNe 2003bn and 2013ej, but with a lower error as we use more $\mathrm{SNe}$ to determine the $\mathrm{ZP}_{\mathrm{C} 3}$ value.

The expression for the $E_{B-V}^{\mathrm{h}}$ error is

$\sigma_{E_{B-V}^{\mathrm{h}}}=\sqrt{\hat{\sigma}_{A, k}^{2}+\sigma_{\mathrm{ZP}}^{2}+\sigma_{m}^{2}}$

where $\hat{\sigma}_{A, k}$ is given by equation (D6), and

$\sigma_{m}=\left|\frac{E_{B-V}^{\mathrm{h}}-\left\langle(B-V)_{i}\right\rangle+\overline{\left\langle(B-V)_{i}^{\mathrm{ZP}}\right\rangle}}{R_{V}-R_{I}-m_{\mathrm{C} 3}}\right| \sigma_{m_{\mathrm{C} 3}}$

is the error induced by the $m_{\mathrm{C} 3}$ uncertainty. In the latter expression, $\overline{\left\langle(B-V)_{i}^{\mathrm{ZP}}\right\rangle}=1.065$ is the mean $\left\langle(B-V)_{i}^{\mathrm{ZP}}\right\rangle$ value of the five $\mathrm{SNe}$ we use to calibrate the C3 method.

For example, Fig. $\mathrm{F} 1$ shows the $E_{B-V, i}^{\mathrm{h}}$ estimates for SN 2009N obtained with the C3 method. For that SN we compute $E_{B-V}^{\mathrm{h}}=0.277 \pm 0.082 \mathrm{mag}$.
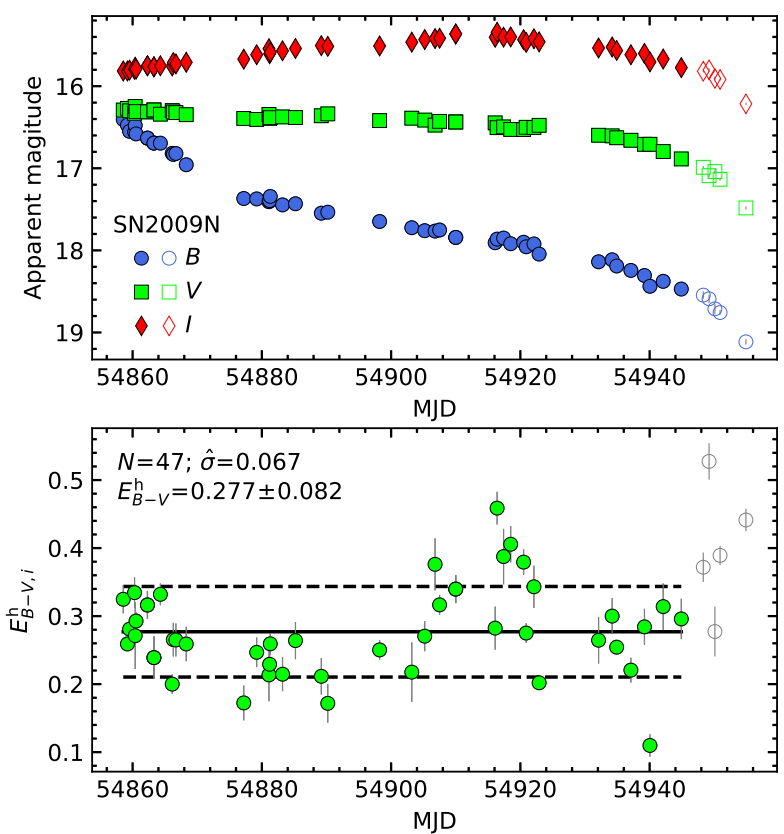

Figure F1. Top panel: $B V I$ light curves of SN 2009N during the plateau phase. Bottom panel: $E_{B-V, i}^{\mathrm{h}}$ estimates obtained with the C3 method. Filled symbols are the data used to compute $E_{B-V}^{\mathrm{h}}$. The solid line corresponds to the $E_{B-V}^{\mathrm{h}}$ value that maximizes the likelihood, and dashed lines are the $\pm 1 \hat{\sigma}$ limits around $E_{B-V}^{\mathrm{h}}$. Error bars are $1 \sigma$ errors due to uncertainties on photometry.

\section{APPENDIX G: SPECTROSCOPIC REDDENING}

Let a set of $N$ SN spectra $\left\{f_{i}\right\}$ corrected for $E_{B-V}^{\mathrm{G}}$ and $z_{\text {helio }}^{\mathrm{SN}}$, where each spectrum has a dispersion $\Delta_{i}$ (in $\AA /$ pixel) and $n_{i}$ flux measurements at wavelengths $\lambda_{i, k}\left(k=1, \ldots, n_{i}\right)$, and a set of $M$ reddening-free spectra $\left\{F_{j}\right\}$. For each $\left\{f_{i}, F_{j}\right\}$ combination, the $E_{B-V}^{\mathrm{h}}$ value can be inferred minimizing

$\hat{\sigma}_{i, j}^{2}=\frac{\Delta_{i}^{-1}}{n_{i}-2} \sum_{k}^{n_{i}}\left[\log \left[\frac{f_{i}\left(\lambda_{i, k}\right)}{F_{j}\left(\lambda_{i, k}\right)}\right]-A+\frac{E_{B-V}^{\mathrm{h}} R_{\lambda_{i, k}}}{2.5}\right]^{2}$.

Here $A$ is a constant accounting for differences in SN distances and photosphere radius sizes, and $R_{\lambda}$ is the extinction curve for the host-galaxy dust along the line of sight. The best estimation for $E_{B-V}^{\mathrm{h}}$ is that provided by the $\left\{f_{i}, F_{j}\right\}$ combination with the lowest $\hat{\sigma}_{i, j}$ value.

In this work we use the D13 and L17 spectral models as $\left\{F_{j}\right\}$, and adopt the extinction curve of Fitzpatrick (1999) with $R_{V}=3.1$. For an input SN we select spectra: (1) earlier that $40 \mathrm{~d}$ since the explosion, (2) with a minimum wavelength coverage of $360-700 \mathrm{~nm}$, and (3) with differences between synthetic and observed colours lower than $0.1 \mathrm{mag}$. These criteria are such that the selected spectra are suitable to measure $E_{B-V}^{\mathrm{h}}$ with an error of $0.05 \mathrm{mag}$ (e.g. Olivares E. et al. 2010). To be conservative, we adopt an error of $0.1 \mathrm{mag}$. For example, Fig. G1 shows the $\left\{f_{i}, F_{j}\right\}$ combination for SN $2009 \mathrm{~N}$ that minimizes equation (G1). For that $\mathrm{SN}$ we compute $E_{B-V}^{\mathrm{h}}=0.202$ mag. 


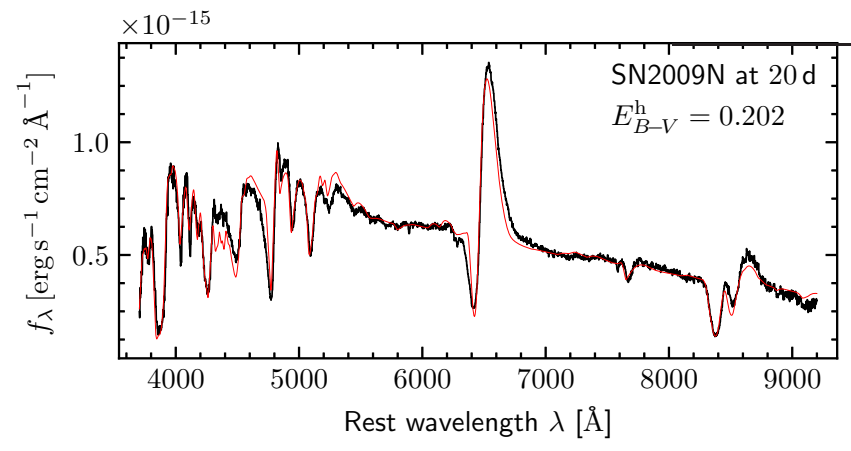

Figure G1. Spectrum of SN 2009N (corrected for $E_{B-V}^{\mathrm{G}}$ and $z_{\text {helio }}^{\mathrm{SN}}$, black line) and model (reddened for $E_{B-V}^{\mathrm{h}}=0.202 \mathrm{mag}$, red line) that minimizes equation (G1).

\section{APPENDIX H: MAGNITUDE TRANSFORMATIONS}

We present magnitude transformations between Sloan and Johnson-Kron-Cousins filters, and between $r_{\text {ZTF }}$ and $R$. All magnitudes are corrected for $E_{B-V}^{\mathrm{G}}, E_{B-V}^{\mathrm{h}}$, and $K$-correction, while reported uncertainties are $1 \hat{\sigma}$ errors.

Using $12 \mathrm{SNe}$ in out set having $V R I$ and $r i$ photometry in the radioactive tail (see Table L1), we find that $r-R$ and $i-I$ have a small linear dependence on $V-I$, i.e.,

$c_{y}=a+b(V-I)$,

where $c_{y}=r-R, i-I$. Fig. H1 shows the mean $r-R(\langle r-R\rangle)$ and $i-I(\langle i-I\rangle)$ values of each $\mathrm{SN}$ as a function of the mean $V-I(\langle V-I\rangle)$. The average of equation (H1) is $\left\langle c_{y}\right\rangle=$ $a+b\langle V-I\rangle$, so we can compute $a$ and $b$ fitting a straight line to the data plotted in the figure. We obtain

$r-R=0.17(V-I)-0.03 \pm 0.08$

and

$i-I=0.12(V-I)+0.38 \pm 0.10$.

Using the same technique for $V-R$ versus $V-I$ during the photospheric phase (69 SNe, Fig. H1), we obtain

$V-R=0.59(V-I)+0.05 \pm 0.05$.

To convert $r$ to $R$ magnitudes near the maximum light, we use $18 \mathrm{SNe}$ with $r R$ photometry at $\Delta t<20 \mathrm{~d}$ (see Table L1). We find that $r-R$ is consistent with a constant value of $r-R=0.12 \pm 0.08 \mathrm{mag}$ (see Fig. H2(a)).

To compute conversions between magnitudes at $\Delta t=50 \mathrm{~d}$ we use 18,25 , and $24 \mathrm{SNe}$ for $g / V, r / R$, and $i / I$ transformations, respectively. We find $(g-V)_{50 \mathrm{~d}}=-0.20+$ $0.61(B-V)_{50 \mathrm{~d}} \pm 0.06$ (see Fig H2(b)), while the $(r-R)_{50 \mathrm{~d}}$ and $(i-I)_{50 \mathrm{~d}}$ values are consistent with constant values of $0.13 \pm 0.05$ and $0.39 \pm 0.06$, respectively (see Fig H2(c)-(d)).

The public ZTF photometry is not in the SDSS photometric system but in the native ZTF one, so we cannot just adopt $r_{\mathrm{ZTF}}=r$. To assess how similar the $r_{\mathrm{ZTF}}$ photometry is to the $r$ - and $R$-band photometry, we compute synthetic magnitudes (Appendix A) using SN spectra. From the spectra of the $\mathrm{SNe}$ in our set, we select those having differences between synthetic and observed $V-r, V-R, V-i$, and/or $V-I$ colours lower than 0.1 mag.

The left-hand panels of Fig. H3 show the $r_{\mathrm{ZTF}}-r$ (top panel) and $r_{\mathrm{ZTF}}-R$ (bottom panel) values, computed with
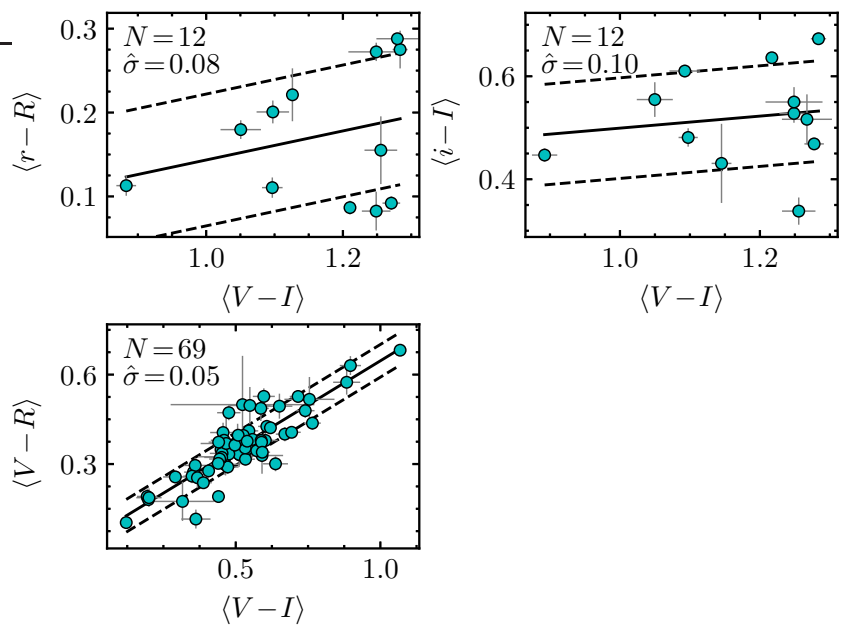

Figure H1. Upper-left (upper-right) panel: mean $r-R(i-I)$ against mean $V-I$ for 12 normal SNe II during the radioactive tail. Bottom panel: mean $V-R$ versus mean $V-I$ for 69 normal SNe II during the photospheric phase. Solid and dashed lines are straight line fits and $\pm 1 \hat{\sigma}$ limits, respectively. Error bars are $1 \sigma$ errors.
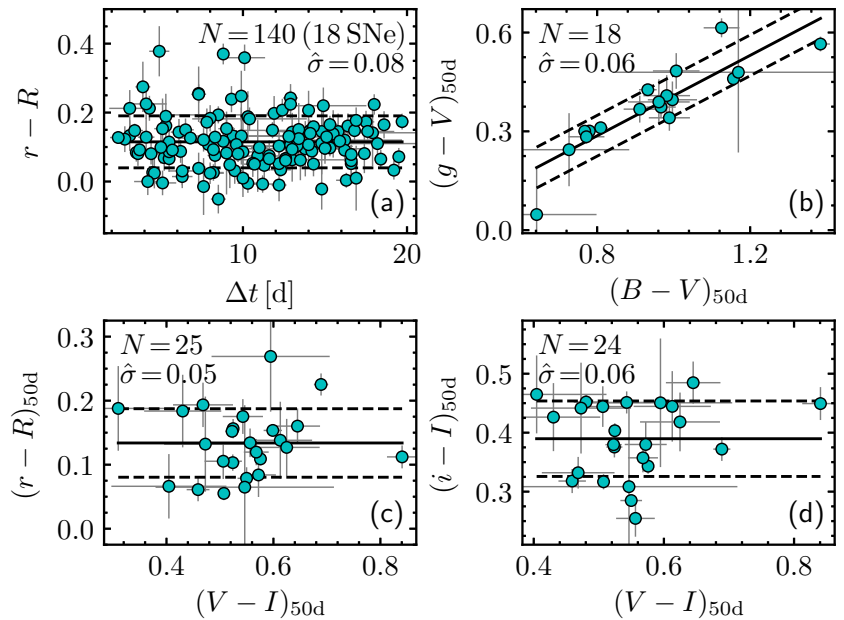

Figure H2. Panel (a): $r-R$ versus time since explosion for $\Delta t<20$ d. Panel (b): $g-V$ against $B-V$ at $\Delta t=50$ d. Panels (c) and (d): $r-R$ and $i-I$ versus $V-I$ at $\Delta t=50 \mathrm{~d}$, respectively. Solid and dashed lines are the best fits and the $\pm 1 \hat{\sigma}$ limits, respectively. Error bars are $1 \sigma$ errors.

308 spectra of 36 SNe during the photospheric and transition phase, against the time since explosion. We see that the $r_{\mathrm{ZTF}}-R$ values have a lower dependence on time and a lower scatter than the $r_{\mathrm{ZTF}}-r$ estimates (the $y$-axis scale is the same in the two panels). Therefore, the $r_{\mathrm{ZTF}}$ light curves during the photospheric and transition phase are more similar to the $R$-band light curves than the $r$-band ones. Given the low $\hat{\sigma}$ value $(0.008 \mathrm{mag})$ around the mean $r_{\mathrm{ZTF}}-R(0.138 \mathrm{mag})$, we consider the time dependence to be negligible and adopt $r_{\mathrm{ZTF}}-R=0.138 \pm 0.008 \mathrm{mag}$ to transform $r_{\text {ZTF }}$ into $R$ magnitudes. We find similar results for the radioactive tail (right-hand panels, using 56 spectra of $13 \mathrm{SNe}$ ), for which we adopt $r_{\mathrm{ZTF}}-R=0.142 \pm 0.024 \mathrm{mag}$.

In the literature we find only one normal SN II having $r_{\text {ZTF }}$ and $R$ photometry (SN 2018aoq; Tsvetkov et al. 2019). 

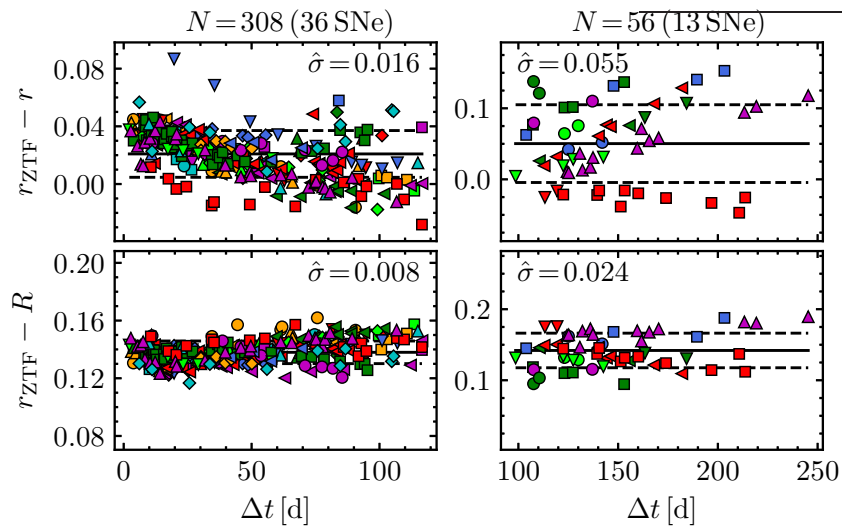

Figure H3. $r_{\mathrm{ZTF}}-r$ (top panels) and $r_{\mathrm{ZTF}}-R$ (bottom panels) values computed from spectra of normal SNe II in the photospheric and transition phase (left-hand panels) and in the radioactive tail (right-hand panels), against the time since explosion. Solid and dashed lines are mean values and $\pm 1 \hat{\sigma}$ limits, respectively.

For that SN we measure a mean $r_{\mathrm{ZTF}}-R$ of $0.17 \pm 0.04 \mathrm{mag}$ (16 points in the photospheric phase), which is consistent within its error with our result from synthetic magnitudes.

\section{APPENDIX I: $K$-CORRECTIONS}

We express the $x$-band $K$-correction $\left(K_{x, j}\right)$ such that the apparent magnitude $\left(m_{x, j}\right)$ corrected for $K$-correction is $m_{x, j}^{K}=m_{x, j}-K_{x, j}$, where

$K_{x, j}=-2.5 \log \left(1+z_{\text {helio }}^{\mathrm{SN}}\right)+k_{x, j}^{s}$,

being

$k_{x, j}^{s}=2.5 \log \left(\frac{d \lambda S_{x, \lambda} \lambda f_{j, \lambda}}{d \lambda^{\prime} S_{x, \lambda} \lambda^{\prime} f_{j, \lambda^{\prime}}}\right)$

and $\lambda^{\prime}=\lambda /\left(1+z_{\text {helio }}^{\mathrm{SN}}\right)$. To compute $k_{x}^{s}$ we use the spectral

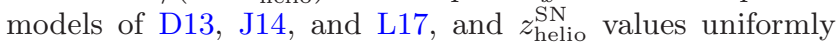
distributed between 0.001 and 0.043 . Expressing $k_{x}^{s}$ as polynomials on $\Delta t$, we obtain

$k_{x, j}^{s}=\left(\sum_{i=0} k_{i} \cdot\left[\frac{\Delta t_{j}}{100 \mathrm{~d}}\right]^{i} \pm \hat{\sigma}\right) z_{\text {helio }}^{\mathrm{SN}}$.

Table I1 lists the parameters for the photospheric and radioactive tail phase. At $\Delta t=200 \mathrm{~d}$ (the midpoint of the time range we use to estimate $\left.M_{56 \mathrm{Ni}}\right) K_{x}$ values are $<0.03 \mathrm{mag}$ for $z_{\text {helio }}^{\mathrm{SN}}=0.009$ (the median redshift in our set), while for $z_{\text {helio }}^{\mathrm{SN}}>0.02$ (15 out of $110 \mathrm{SNe}$ in our sample) $K_{x}$ values start to be comparable to the typical photometry errors.

\section{APPENDIX J: INDIRECT STEEPNESS VALUE}

In order to calculate $S_{x}$ indirectly from steepnesses in bands other than $x$, we compute steepness transformations.

Fig. J1 shows $S_{V}$ against the steepness for grRiI bands. Using the model selection procedure (Appendix C), we find that the five correlations plotted in the figure are well represented by straight lines. In general, for the 30 possible combinations with the $g \operatorname{VrRiI}$ bands, we find that correlations between the $x$ - and $b$-band steepness are given by

$S_{x}=c_{x, b}+d_{x, b} S_{b}$.
Table I1. $K$-correction parameters.

\begin{tabular}{lccccccc}
\hline$x$ & $k_{0}^{*}$ & $k_{1}^{*}$ & $k_{2}^{*}$ & $\hat{\sigma}^{*}$ & $k_{0}^{\dagger}$ & $k_{1}^{\dagger}$ & $\hat{\sigma}^{\dagger}$ \\
\hline$B$ & -1.50 & 19.8 & -10.8 & 1.32 & - & - & - \\
$g$ & -1.23 & 16.1 & -10.2 & 0.90 & - & - & - \\
$V$ & -1.46 & 8.85 & -5.27 & 0.66 & 8.10 & -1.69 & 2.97 \\
$r$ & -0.05 & 6.56 & -4.12 & 0.72 & 4.12 & 0.88 & 2.21 \\
$r_{\text {ZTF }}$ & -0.70 & 4.21 & -2.54 & 0.24 & 0.60 & 0.77 & 1.22 \\
$R$ & -0.42 & 3.56 & -2.09 & 0.21 & 1.48 & 0.57 & 0.92 \\
$i$ & -1.05 & 2.33 & -0.87 & 0.37 & 1.05 & 0.26 & 1.00 \\
$I$ & -1.00 & 3.60 & -1.50 & 0.24 & 6.31 & -1.69 & 1.07 \\
\hline \multicolumn{7}{l}{ Note. Parameters for the photospheric $(*)$ and radioactive tail }
\end{tabular}

(†) phase.

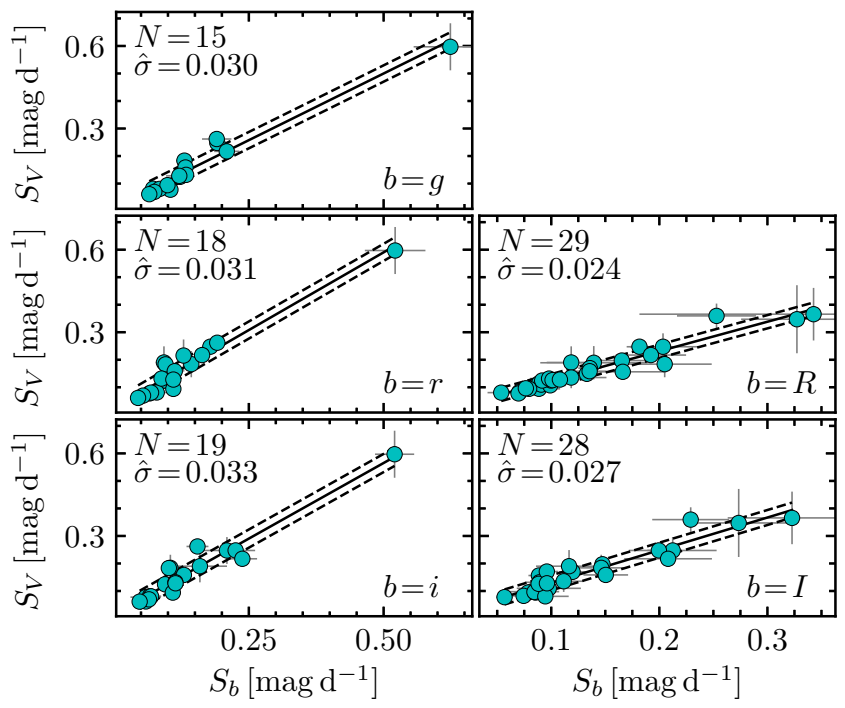

Figure J1. $V$-band steepness against the steepness for the grRiI bands. Solid lines are straight-line fits to the data, while dashed ones are $\pm 1 \hat{\sigma}$ limits around the fits. Error bars are $1 \sigma$ errors.

Table L8 reports the $c_{x, b}$ and $d_{x, b}$ parameters, their bootstrap errors, and the $\hat{\sigma}_{x, b}$ values.

For an SN with a set of $n$ steepness estimates, $\left\{S_{b}\right\}_{b \neq x}$, we can compute a set of $n S_{x}$ values, $\left\{S_{x}\right\}$, using equation (J1). Our best indirect measurement of $S_{x}$ will be the weighted mean of the $\left\{S_{x}\right\}$ values, $S_{x}^{*}$, given by

$S_{x}^{*}=\frac{1}{\sum_{b \neq x} w_{x, b}} \sum_{b \neq x}\left(c_{x, b}+d_{x, b} S_{b}\right) w_{x, b}$

where $w_{x, b}=\left[d_{x, b}^{2} \sigma_{S_{b}}^{2}+\hat{\sigma}_{x, b}^{2}\right]^{-1}$. The error on $S_{x}^{*}$ is simply

$\sigma_{S_{x}^{*}}=\sqrt{\frac{1}{\sum_{b \neq x} w_{x, b}}}$.

\section{APPENDIX K: REFERENCE LIST FOR THE DATA}

Here the references for Tables L1, L2, L3, and L4.

(1) Barbon et al. (1982); (2) Buta (1982); (3)

Tsvetkov (1983); (4) Pennypacker et al. (1989); (5)

Ruiz-Lapuente et al. (1990); (6) Benetti et al. (1991);

(7) Turatto et al. (1993); (8) Schmidt et al. (1993); (9)

Benetti et al. (1994); (10) Cappellaro et al. (1995); (11)

Blanton et al. (1995); (12) Galbany et al. (2016); (13)

Clocchiatti et al. (1996); (14) Pastorello et al. (2004);

(15) Inserra et al. (2013); (16) Benetti et al. (2001); (17) 
Elmhamdi et al. (2003a); (18) Krisciunas et al. (2009); (19) Faran et al. (2014a); (20) Rubenstein (1999); (21) Pastorello et al. (2009b); (22) Leonard et al. (2002); (23) Pozzo et al. (2006); (24) Tsvetkov et al. (2007); (25) Spiro et al. (2014); (26) Hendry et al. (2005); (27) Faran et al. (2014b); (28) Gurugubelli et al. (2008); (29) Zhang et al. (2006); (30) Vinkó et al. (2006); (31) Vinkó et al. (2009); (32) Meikle et al. (2011); (33) Anderson et al. (2014); (34) Sahu et al. (2006); (35) Misra et al. (2007); (36) Maguire et al. (2010); (37) Arcavi et al. (2012); (38) Tsvetkov et al. (2006); (39) Dessart et al. (2008); (40) Pastorello et al. (2009a); (41) Hicken et al. (2017); (42) Brown et al. (2009); (43) Andrews et al. (2011); (44) Andrews et al. (2010); (45) Inserra et al. (2011); (46) de Jaeger et al. (2019); (47) Van Dyk et al. (2012); (48) Van Dyk (2013); (49) Pignata (2013); (50) Roy et al. (2011a); (51) Roy et al. (2011b); (52) Takáts et al. (2014); (53) Tsvetkov (2014); (54) Inserra et al. (2012); (55) Elias-Rosa et al. (2011); (56) Takáts et al. (2015); (57) Fraser et al. (2011); (58) Rubin et al. (2016); (59) Tomasella et al. (2013); (60) Bayless et al. (2013); (61) Bose et al. (2013); (62) Munari et al. (2013); (63) Dall'Ora et al. (2014); (64) Barbarino et al. (2015); (65) Tomasella et al. (2018); (66) Bose et al. (2015a); (67) Zhang et al. (2014); (68) Valenti et al. (2016); (69) Valenti et al. (2015); (70) Richmond (2014); (71) Bose et al. (2015b); (72) Huang et al. (2015); (73) Dhungana et al. (2016); (74) Yuan et al. (2016); (75) Bullivant et al. (2018); (76) Bose et al. (2016); (77) Terreran et al. (2016); (78) Huang et al. (2016); (79) Dastidar et al. (2021); (80) Singh et al. (2018); (81) Wyrzykowski et al. (2014); (82) Dastidar et al. (2019); (83) Dastidar et al. (2018); (84) Bostroem et al. (2019); (85) Huang et al. (2018); (86) Bose et al. (2019); (87) Müller-Bravo et al. (2020); (88) Nakaoka et al. (2018); (89) Hosseinzadeh et al. (2018); (90) Singh et al. (2019); (91) Tartaglia et al. (2018); (92) Afsariardchi et al. (2019); (93) Tartaglia et al. (2021); (94) Rho et al. (2018); (95) Tsvetkov et al. (2018); (96) Tinyanont et al. (2019); (97) Van Dyk et al. (2019); (98) Szalai et al. (2019); (99) Buta \& Keel (2019); (100) Andrews et al. (2019); (101) Dong et al. (2020); (102) Reguitti et al. (2021); (103) Anand et al. (2018); (104) de Jaeger et al. (2020); (105) Riess et al. (2016); (106) McQuinn et al. (2017); (107) Freedman et al. (2019); (108) Saha et al. (2006); (109) Carlin et al. (2016); (110) Monachesi et al. (2016); (111) Zgirski et al. (2017); (112) Jang \& Lee (2017); (113) Rizzi et al. (2007); (114) Tikhonov \& Galazutdinova (2020); (115) Hamuy (2001); (116) Rodríguez et al. (2014); (117) Olivares E. et al. (2010); (118) Turatto et al. (1998); (119) Pastorello et al. (2006); (120) Quimby et al. (2007); (121) Li et al. (2007); (122) Yaron et al. (2017); (123) Childress et al. (2016); (124) Thompson (1982); (125) Gutiérrez et al. (2017a); (126) Harutyunyan et al. (2008); (127) Hendry et al. (2006); (128) Shivvers et al. (2017); (129) Liao et al. (2019); (130) Itagaki (2016); (131) Dimai (2016); (132) Wiggins (2017).

\section{APPENDIX L: TABLES}

This paper has been typeset from a $\mathrm{TEX}_{\mathrm{E}} / \mathrm{LAT}_{\mathrm{E}} \mathrm{X}$ file prepared by the author. 
Table L1. SN sample.

\begin{tabular}{|c|c|c|c|c|c|c|c|}
\hline $\mathrm{SN}$ & Host galaxy & $\begin{array}{l}E_{B-V}^{G} \\
(\mathrm{mag})\end{array}$ & $\begin{array}{c}c z_{\text {helio }}^{\text {SN }} \\
\left(\mathrm{km} \mathrm{s}^{-1}\right)\end{array}$ & $\begin{array}{c}\mu \\
(\mathrm{mag})\end{array}$ & $\begin{array}{l}E_{B-V}^{\mathrm{h}} \\
(\mathrm{mag})\end{array}$ & $\begin{array}{c}t_{0} \\
(\mathrm{MJD})\end{array}$ & References \\
\hline $1980 \mathrm{~K}$ & NGC 6946 & 0.291 & 40 & $29.44 \pm 0.09$ & $-0.106 \pm 0.084$ & $44528.2 \pm 5.9$ & $1,2,3$ \\
\hline 1986I & M99 & 0.034 & 2407 & $30.71 \pm 0.40$ & $0.212 \pm 0.142$ & $46556.4 \pm 1.9$ & 4 \\
\hline $1988 \mathrm{~A}$ & M58 & 0.035 & 1517 & $31.17 \pm 0.35$ & $0.090 \pm 0.111$ & $47176.2 \pm 0.3$ & $5,6,7$ \\
\hline $1990 \mathrm{E}$ & NGC 1035 & 0.022 & 1362 & $30.83 \pm 0.26$ & $0.598 \pm 0.072$ & $47934.4 \pm 1.4$ & 8,9 \\
\hline $1990 \mathrm{~K}$ & NGC 150 & 0.012 & 1584 & $31.57 \pm 0.24$ & $0.227 \pm 0.034$ & $48013.8 \pm 4.2$ & 10 \\
\hline $1991 \mathrm{G}$ & NGC 4088 & 0.017 & 757 & $30.76 \pm 0.17$ & $0.025 \pm 0.071$ & $48281.5 \pm 5.3$ & 11 \\
\hline 1991al & LEDA 140858 & 0.044 & 4572 & $32.80 \pm 0.12$ & $0.067 \pm 0.026$ & $48446.7 \pm 3.7$ & 12 \\
\hline $1992 \mathrm{H}$ & NGC 5377 & 0.015 & 1793 & $32.07 \pm 0.17$ & $0.167 \pm 0.123$ & $48656.4 \pm 4.5$ & 13 \\
\hline $1992 \mathrm{ba}$ & NGC 2082 & 0.050 & 1135 & $30.78 \pm 0.29$ & $0.096 \pm 0.029$ & $48886.1 \pm 2.8$ & 12 \\
\hline $1994 \mathrm{~N}$ & UGC 5695 & 0.032 & 2940 & $33.24 \pm 0.20$ & $0.045 \pm 0.036$ & $49453.9 \pm 4.5$ & 14 \\
\hline $1995 \mathrm{ad}^{\dagger}$ & NGC 2139 & 0.029 & 1837 & $32.24 \pm 0.18$ & $0.090 \pm 0.111$ & $49972.0 \pm 4.2$ & 15 \\
\hline 1996W & NGC 4027 & 0.036 & 1617 & $31.86 \pm 0.19$ & $0.260 \pm 0.054$ & $50180.2 \pm 2.5$ & 15 \\
\hline 1997D & NGC 1536 & 0.017 & 1217 & $30.93 \pm 0.25$ & $0.090 \pm 0.111$ & $50361.0 \pm 15.0$ & 16 \\
\hline 1999ca & NGC 3120 & 0.094 & 2791 & $32.82 \pm 0.11$ & $0.076 \pm 0.033$ & $51273.4 \pm 2.9$ & 12 \\
\hline $1999 \mathrm{em}^{\dagger}$ & NGC 1637 & 0.035 & 800 & $30.31 \pm 0.09$ & $0.082 \pm 0.034$ & $51474.5 \pm 2.0$ & $12,17,18,19$ \\
\hline 1999ga & NGC 2442 & 0.173 & 1466 & $31.51 \pm 0.05$ & $0.511 \pm 0.084$ & $51419.5 \pm 20.0$ & 20,21 \\
\hline 1999gi & NGC 3184 & 0.014 & 503 & $30.40 \pm 0.18$ & $0.250 \pm 0.033$ & $51518.6 \pm 1.8$ & 19,22 \\
\hline $2001 X$ & NGC 5921 & 0.034 & 1480 & $31.52 \pm 0.21$ & $0.063 \pm 0.031$ & $51963.6 \pm 2.6$ & 19 \\
\hline $2001 \mathrm{dc}$ & NGC 5777 & 0.009 & 2145 & $32.69 \pm 0.16$ & $0.694 \pm 0.076$ & $52046.0 \pm 5.0$ & 14 \\
\hline $2002 \mathrm{gw}$ & NGC 922 & 0.016 & 3143 & $32.98 \pm 0.22$ & $0.138 \pm 0.036$ & $52554.8 \pm 3.0$ & 12 \\
\hline $2002 \mathrm{hh}$ & NGC 6946 & 1.065 & 110 & $29.44 \pm 0.09$ & $1.545 \pm 0.182$ & $52575.6 \pm 2.5$ & 23,24 \\
\hline $2002 \mathrm{hx}$ & PGC 23727 & 0.045 & 9299 & $35.53 \pm 0.08$ & $0.140 \pm 0.043$ & $52580.3 \pm 3.7$ & 12 \\
\hline 2003B & NGC 1097 & 0.023 & 1141 & $30.62 \pm 0.25$ & $0.023 \pm 0.033$ & $52622.2 \pm 4.2$ & 12 \\
\hline $2003 \mathrm{~T}$ & UGC 4864 & 0.027 & 8368 & $35.33 \pm 0.10$ & $0.154 \pm 0.040$ & $52652.6 \pm 4.6$ & 12 \\
\hline $2003 Z$ & NGC 2742 & 0.033 & 1289 & $31.62 \pm 0.23$ & $0.109 \pm 0.050$ & $52665.1 \pm 2.4$ & 19,25 \\
\hline $2003 \mathrm{fb}$ & UGC 11522 & 0.155 & 5081 & $34.05 \pm 0.13$ & $0.371 \pm 0.046$ & $52779.0 \pm 4.4$ & 12 \\
\hline $2003 \mathrm{gd}$ & M74 & 0.060 & 657 & $29.95 \pm 0.08$ & $0.144 \pm 0.040$ & $52716.5 \pm 21.0$ & $12,19,26$ \\
\hline 2003hd & ESO $543-G 17$ & 0.011 & 12031 & $35.97 \pm 0.08$ & $0.124 \pm 0.042$ & $52857.2 \pm 2.8$ & 12 \\
\hline $2003 \mathrm{hk}$ & NGC 1085 & 0.032 & 6880 & $34.77 \pm 0.12$ & $0.142 \pm 0.054$ & $52868.3 \pm 2.7$ & 12,27 \\
\hline $2003 \mathrm{hn}^{\dagger}$ & NGC 1448 & 0.012 & 1305 & $31.31 \pm 0.04$ & $0.169 \pm 0.040$ & $52865.6 \pm 4.0$ & 12,18 \\
\hline 2003 ho & ESO $235-G 58$ & 0.033 & 4091 & $33.70 \pm 0.18$ & $0.640 \pm 0.050$ & $52848.5 \pm 3.3$ & 12 \\
\hline 2003iq & NGC 772 & 0.062 & 2331 & $32.41 \pm 0.16$ & $0.122 \pm 0.038$ & $52919.4 \pm 0.9$ & 12,19 \\
\hline $2004 \mathrm{~A}$ & NGC 6207 & 0.013 & 852 & $30.87 \pm 0.26$ & $0.177 \pm 0.043$ & $53012.5 \pm 1.7$ & 28 \\
\hline $2004 \mathrm{dj}^{\dagger}$ & NGC 2403 & 0.034 & 221 & $27.46 \pm 0.11$ & $0.094 \pm 0.035$ & $53180.6 \pm 15.6$ & $29,30,31,32$ \\
\hline 2004 eg & UGC 3053 & 0.390 & 2414 & $32.46 \pm 0.24$ & $0.160 \pm 0.150$ & $53169.5 \pm 30.0$ & 25 \\
\hline 2004 ej & NGC 3095 & 0.060 & 2723 & $32.76 \pm 0.13$ & $0.145 \pm 0.116$ & $53231.5 \pm 4.2$ & 33 \\
\hline $2004 \mathrm{et}^{\dagger}$ & NGC 6946 & 0.293 & 40 & $29.44 \pm 0.09$ & $0.073 \pm 0.043$ & $53270.5 \pm 0.3$ & $19,34,35,36$ \\
\hline $2004 f x$ & MCG -02-14-3 & 0.088 & 2673 & $32.73 \pm 0.19$ & $0.090 \pm 0.115$ & $53305.1 \pm 1.4$ & 33 \\
\hline 2005af & NGC 4945 & 0.159 & 563 & $27.75 \pm 0.12$ & $0.090 \pm 0.117$ & $53320.8 \pm 17.0$ & 33 \\
\hline $2005 \mathrm{au}$ & NGC 5056 & 0.010 & 5592 & $34.43 \pm 0.12$ & $0.117 \pm 0.073$ & $53441.4 \pm 6.1$ & 37 \\
\hline 2005ay & NGC 3938 & 0.018 & 850 & $30.68 \pm 0.21$ & $0.035 \pm 0.037$ & $53450.7 \pm 1.8$ & 19,38 \\
\hline $2005 \operatorname{cs}^{\dagger *}$ & M51a & 0.032 & 463 & $29.67 \pm 0.07$ & $0.124 \pm 0.037$ & $53548.4 \pm 0.3$ & $19,38,39,40$ \\
\hline $2005 d x$ & ESO $550-\mathrm{G} 2$ & 0.021 & 8012 & $35.09 \pm 0.09$ & $0.090 \pm 0.142$ & $53615.7 \pm 4.6$ & 33 \\
\hline $2006 \mathrm{my}$ & NGC 4651 & 0.012 & 788 & $31.40 \pm 0.10$ & $-0.062 \pm 0.152$ & $53942.5 \pm 20.0$ & 36 \\
\hline $2006 \mathrm{ov}^{\ddagger}$ & M61 & 0.019 & 1566 & $31.40 \pm 0.63$ & $0.275 \pm 0.105$ & $53973.5 \pm 6.0$ & 25,41 \\
\hline 2007 aa & NGC 4030 & 0.023 & 1574 & $31.99 \pm 0.27$ & $0.046 \pm 0.100$ & $54131.9 \pm 4.1$ & $33,41,42$ \\
\hline $2007 \mathrm{hv}$ & UGC 2858 & 0.418 & 5054 & $34.05 \pm 0.16$ & $0.000 \pm 0.100$ & $54346.6 \pm 2.8$ & 41 \\
\hline 2007 it & NGC 5530 & 0.099 & 1193 & $30.56 \pm 0.24$ & $0.109 \pm 0.111$ & $54348.0 \pm 0.6$ & 33,43 \\
\hline 2007od & UGC 12846 & 0.031 & 1734 & $31.63 \pm 0.25$ & $0.134 \pm 0.070$ & $54388.2 \pm 4.1$ & $41,44,45,46$ \\
\hline $2008 \mathrm{~K}$ & ESO 504-G4 & 0.033 & 7964 & $35.29 \pm 0.10$ & $0.090 \pm 0.111$ & $54466.8 \pm 4.8$ & 33 \\
\hline $2008 \mathrm{M}$ & ESO $121-G 26$ & 0.039 & 2192 & $32.62 \pm 0.20$ & $0.090 \pm 0.112$ & $54475.3 \pm 3.3$ & 33 \\
\hline 2008aw & NGC 4939 & 0.035 & 3110 & $33.19 \pm 0.17$ & $0.226 \pm 0.043$ & $54519.3 \pm 3.9$ & 33 \\
\hline $2008 \mathrm{bk}^{\dagger}$ & NGC 7793 & 0.017 & 230 & $27.66 \pm 0.08$ & $0.090 \pm 0.111$ & $54547.1 \pm 2.6$ & $47,48,49$ \\
\hline $2008 \mathrm{gz}$ & NGC 3672 & 0.036 & 1862 & $32.22 \pm 0.15$ & $-0.031 \pm 0.040$ & $54693.5 \pm 5.0$ & 50 \\
\hline 2008 in ${ }^{\dagger キ *}$ & M61 & 0.019 & 1566 & $31.40 \pm 0.63$ & $0.037 \pm 0.044$ & $54824.2 \pm 0.1$ & $41,46,51$ \\
\hline $2009 \mathrm{~N}^{\ddagger *}$ & NGC 4487 & 0.018 & 905 & $30.90 \pm 0.38$ & $0.265 \pm 0.045$ & $54850.1 \pm 3.4$ & $41,46,52$ \\
\hline 2009at & NGC 5301 & 0.015 & 1503 & $31.82 \pm 0.22$ & $0.551 \pm 0.079$ & $54899.1 \pm 2.0$ & 46 \\
\hline 2009ay & NGC 6479 & 0.034 & 6650 & $34.74 \pm 0.13$ & $0.252 \pm 0.087$ & $54898.3 \pm 4.4$ & $41,46,53$ \\
\hline $2009 \mathrm{bw}^{\dagger}$ & UGC 2890 & 0.197 & 1155 & $31.07 \pm 0.26$ & $0.160 \pm 0.049$ & $54916.5 \pm 3.0$ & 54 \\
\hline $2009 \mathrm{dd}$ & NGC 4088 & 0.017 & 757 & $30.76 \pm 0.17$ & $0.285 \pm 0.057$ & $54916.0 \pm 4.2$ & 15,41 \\
\hline 2009hd & M66 & 0.029 & 727 & $30.15 \pm 0.07$ & $1.206 \pm 0.056$ & $55001.6 \pm 3.9$ & 55 \\
\hline $2009 \mathrm{ib}^{*}$ & NGC 1559 & 0.026 & 1304 & $31.41 \pm 0.05$ & $0.147 \pm 0.038$ & $55040.3 \pm 4.0$ & 56 \\
\hline $2009 \mathrm{md}^{\dagger}$ & NGC 3389 & 0.023 & 1308 & $32.06 \pm 0.12$ & $0.165 \pm 0.048$ & $55161.9 \pm 7.9$ & 57 \\
\hline 2010aj & MCG -01-32-35 & 0.029 & 6497 & $34.78 \pm 0.12$ & $0.082 \pm 0.048$ & $55261.8 \pm 3.5$ & 15,41 \\
\hline PTF10gva & NSA 49030 & 0.026 & 8253 & $35.33 \pm 0.10$ & $0.111 \pm 0.111$ & $55320.3 \pm 0.9$ & 58 \\
\hline $2011 \mathrm{fd}$ & NGC 2273B & 0.063 & 2101 & $32.41 \pm 0.22$ & $0.090 \pm 0.111$ & $55777.9 \pm 4.5$ & 46 \\
\hline PTF11go & MCG +09-19-105 & 0.010 & 8037 & $35.22 \pm 0.10$ & $0.160 \pm 0.150$ & $55570.9 \pm 1.4$ & 58 \\
\hline PTF11htj & SDSS J211603.12+123124.4 & 0.060 & 5096 & $34.04 \pm 0.15$ & $0.160 \pm 0.150$ & $55751.9 \pm 1.5$ & 58 \\
\hline PTF11izt & anonymous & 0.047 & 5996 & $34.52 \pm 0.11$ & $0.160 \pm 0.150$ & $55766.8 \pm 1.3$ & 58 \\
\hline $2012 \mathrm{~A}^{*}$ & NGC 3239 & 0.027 & 753 & $30.66 \pm 0.24$ & $0.040 \pm 0.049$ & $55929.3 \pm 2.6$ & 46,59 \\
\hline 2012 aw $^{*}$ & M95 & 0.024 & 778 & $29.93 \pm 0.05$ & $0.115 \pm 0.041$ & $56002.0 \pm 0.5$ & $46,60,61,62,63$ \\
\hline $2012 \mathrm{br}$ & SDSS J122417.04+185529.4 & 0.031 & 6805 & $34.90 \pm 0.12$ & $0.199 \pm 0.260$ & $56000.9 \pm 0.2$ & 58 \\
\hline $2012 \mathrm{~cd}$ & CGCG 271-34 & 0.025 & 3525 & $33.54 \pm 0.19$ & $0.123 \pm 0.134$ & $56019.4 \pm 1.9$ & 58 \\
\hline $2012 \mathrm{ec}^{\dagger \ddagger *}$ & NGC 1084 & 0.023 & 1407 & $31.07 \pm 0.25$ & $0.102 \pm 0.048$ & $56144.5 \pm 3.4$ & 46,64 \\
\hline PTF12grj & WISEA J012039.07+044621.5 & 0.024 & 10193 & $35.62 \pm 0.09$ & $0.160 \pm 0.150$ & $56123.4 \pm 0.6$ & 58 \\
\hline PTF12hsx & SDSS J005503.33+421954.0 & 0.079 & 5696 & $34.34 \pm 0.14$ & $0.167 \pm 0.221$ & $56113.0 \pm 0.5$ & 58 \\
\hline $2013 \mathrm{~K}$ & ESO 9-G10 & 0.122 & 2418 & $32.48 \pm 0.21$ & $0.512 \pm 0.066$ & $56294.5 \pm 4.2$ & 65 \\
\hline $2013 a b^{\ddagger *}$ & NGC 5669 & 0.023 & 1368 & $31.38 \pm 0.20$ & $0.028 \pm 0.040$ & $56339.6 \pm 0.6$ & 46,66 \\
\hline $2013 \mathrm{am}^{*}$ & M65 & 0.021 & 1114 & $30.36 \pm 0.29$ & $0.536 \pm 0.069$ & $56371.8 \pm 0.4$ & $46,65,67$ \\
\hline 2013bu & NGC 7331 & 0.078 & 440 & $30.84 \pm 0.10$ & $0.523 \pm 0.062$ & $56396.6 \pm 2.0$ & 46,68 \\
\hline
\end{tabular}


Table L1 - continued

\begin{tabular}{|c|c|c|c|c|c|c|c|}
\hline $\mathrm{SN}$ & Host galaxy & $\begin{array}{l}E_{B-V}^{G} \\
(\mathrm{mag})\end{array}$ & $\begin{array}{c}c z_{\text {helio }}^{\text {SN }} \\
\left(\mathrm{km} \mathrm{s}^{-1}\right)\end{array}$ & $\begin{array}{c}\mu \\
(\mathrm{mag})\end{array}$ & $\begin{array}{l}E_{B-V}^{\mathrm{h}} \\
(\mathrm{mag})\end{array}$ & $\begin{array}{c}t_{0} \\
\text { (MJD) }\end{array}$ & References \\
\hline 2013by & ESO $138-G 10$ & 0.188 & 1144 & $30.46 \pm 0.29$ & $0.196 \pm 0.100$ & $56401.6 \pm 3.6$ & 69 \\
\hline $2013 \mathrm{ej}^{\dagger \ddagger *}$ & M74 & 0.060 & 657 & $29.95 \pm 0.08$ & $0.044 \pm 0.040$ & $56496.8 \pm 0.2$ & $46,70,71,72,73,74$ \\
\hline $2013 \mathrm{fs}^{*}$ & NGC 7610 & 0.035 & 3554 & $33.32 \pm 0.18$ & $0.027 \pm 0.037$ & $56570.8 \pm 0.5$ & 68,75 \\
\hline $2013 \mathrm{hj}$ & MCG -02-24-3 & 0.045 & 2072 & $32.51 \pm 0.13$ & $0.047 \pm 0.039$ & $56636.8 \pm 0.9$ & 76 \\
\hline iPTF13dkz & SDSS J013611.64+333703.6 & 0.039 & 4797 & $33.85 \pm 0.17$ & $0.144 \pm 0.180$ & $56547.9 \pm 0.3$ & 58 \\
\hline LSQ13dpa & LCSB S1492O & 0.032 & 7045 & $35.00 \pm 0.11$ & $0.160 \pm 0.150$ & $56642.2 \pm 2.0$ & 68 \\
\hline $2014 \mathrm{G}^{\dagger \ddagger *}$ & NGC 3448 & 0.010 & 1160 & $31.96 \pm 0.14$ & $0.268 \pm 0.046$ & $56669.3 \pm 0.8$ & $46,76,77$ \\
\hline $2014 \mathrm{cx} \ddagger *$ & NGC 337 & 0.096 & 1646 & $31.51 \pm 0.22$ & $-0.021 \pm 0.045$ & $56901.9 \pm 0.3$ & 78 \\
\hline $2014 \mathrm{dw}$ & NGC 3568 & 0.092 & 2444 & $32.39 \pm 0.13$ & $0.182 \pm 0.127$ & $56957.5 \pm 10.0$ & 68 \\
\hline ASASSN-14dq & UGC 11860 & 0.060 & 3125 & $32.97 \pm 0.21$ & $0.108 \pm 0.038$ & $56841.0 \pm 5.5$ & 80 \\
\hline ASASSN-14ha & NGC 1566 & 0.008 & 1504 & $30.86 \pm 0.15$ & $0.090 \pm 0.111$ & $56909.5 \pm 0.6$ & 68 \\
\hline OGLE14-18 & ESO $87-G 3$ & 0.049 & 8082 & $35.18 \pm 0.10$ & $0.144 \pm 0.137$ & $56701.7 \pm 0.9$ & 81 \\
\hline $2015 \mathrm{~V}$ & UGC 11000 & 0.031 & 1369 & $31.63 \pm 0.22$ & $0.031 \pm 0.060$ & $57112.3 \pm 4.3$ & 46 \\
\hline $2015 \mathrm{~W}$ & UGC 3617 & 0.118 & 3984 & $33.70 \pm 0.19$ & $0.157 \pm 0.063$ & $57015.0 \pm 6.5$ & 46,68 \\
\hline 2015an & IC 2367 & 0.009 & 2448 & $32.79 \pm 0.13$ & $0.114 \pm 0.111$ & $57268.0 \pm 1.6$ & 82 \\
\hline 2015ba & IC 1029 & 0.015 & 2383 & $32.80 \pm 0.16$ & $0.416 \pm 0.047$ & $57347.5 \pm 4.9$ & 83 \\
\hline $2015 \mathrm{cz}$ & NGC 582 & 0.045 & 4352 & $33.68 \pm 0.16$ & $0.472 \pm 0.065$ & $57290.4 \pm 7.5$ & 79 \\
\hline 2016aqf & NGC 2101 & 0.047 & 1204 & $31.01 \pm 0.25$ & $0.180 \pm 0.100$ & $57442.6 \pm 0.3$ & 87 \\
\hline $2016 \mathrm{bkv}^{*}$ & NGC 3184 & 0.014 & 592 & $30.40 \pm 0.18$ & $0.036 \pm 0.078$ & $57467.5 \pm 1.2$ & 88,89 \\
\hline 2016gfy & NGC 2276 & 0.086 & 2416 & $32.64 \pm 0.22$ & $0.163 \pm 0.045$ & $57641.3 \pm 2.6$ & 90 \\
\hline 2016ija & NGC 1532 & 0.013 & 1040 & $30.55 \pm 0.17$ & $1.950 \pm 0.150$ & $57712.1 \pm 1.0$ & 91 \\
\hline 2017it & anonymous & 0.029 & 12891 & $36.21 \pm 0.07$ & $-0.087 \pm 0.042$ & $57746.2 \pm 0.7$ & 92 \\
\hline 2017ahn & NGC 3318 & 0.067 & 2775 & $32.84 \pm 0.10$ & $0.233 \pm 0.148$ & $57791.8 \pm 0.5$ & 93 \\
\hline 2017 eaw $^{\dagger \ddagger *}$ & NGC 6946 & 0.293 & 40 & $29.44 \pm 0.09$ & $0.059 \pm 0.037$ & $57886.2 \pm 0.6$ & $94,95,96,97,98,99$ \\
\hline $2017 \mathrm{gmr}^{\dagger \ddagger *}$ & NGC 988 & 0.023 & 1510 & $31.08 \pm 0.19$ & $0.332 \pm 0.045$ & $57999.2 \pm 0.6$ & 100 \\
\hline $2018 \mathrm{cuf}$ & IC 5092 & 0.028 & 3248 & $33.10 \pm 0.19$ & $0.221 \pm 0.100$ & $58291.8 \pm 0.3$ & 101 \\
\hline 2018hwm & IC 2327 & 0.022 & 2684 & $33.08 \pm 0.19$ & $0.150 \pm 0.069$ & $58424.8 \pm 0.9$ & 102 \\
\hline
\end{tabular}

Notes. Column 1: SN names. Column 2: SN host galaxy names, Column 3: Galactic colour excesses with random errors of 16 per cent. Column 4: heliocentric

SN redshifts. Column 5 and 6: host galaxy distance moduli and colour excesses, respectively. Column 7: explosion epochs. Column 8: references for the photometry (codes are in Appendix K).

† Selected to compute BCs.

${ }^{\ddagger}$ With $V R I$ and $r i$ photometry in the radioactive tail.

*Used to compute $r-R$ for $\Delta t<20 \mathrm{~d}$.

Table L2. Host galaxy distance moduli. The full table is available online as supplementary data.

\begin{tabular}{llcccccc}
\hline Host galaxy & SN & $\mu_{\mathrm{CPL}}$ & $\mu_{\mathrm{TRGB}}$ & $\mu_{\mathrm{TF}}$ & $\mu_{\mathrm{HLL}}$ & $\mu_{\mathrm{SVF}}$ & $\mu$ \\
\hline NGC 6946 & $1980 \mathrm{~K}$ & - & $29.44 \pm 0.09^{103}$ & - & - & - & $29.44 \pm 0.09$ \\
& $2002 \mathrm{hh}$ & - & - & - & - & - & $29.44 \pm 0.09$ \\
& $2004 \mathrm{et}$ & - & - & - & - & - & $29.44 \pm 0.09$ \\
\hline
\end{tabular}

Notes. Column 1: SN host galaxy names. Column 2: SN names, Columns 3 and 4: Cepheids and TRGB distances from the literature, respectively. Column 5: Tully-Fisher distances from EDD. Column 6: distances compute with the HubbleLemaître law. Column 6: distances inferred from smoothed velocity fields (Kourkchi et al. 2020). Column 8: adopted distance moduli. References for the data (superscript numbers) are in Appendix K.

Table L3. Host galaxy colour excesses. The full table is available online as supplementary data.

\begin{tabular}{lccccc}
\hline $\mathrm{SN}$ & $E_{B-V}^{\mathrm{h}, \mathrm{C} 3}$ & $E_{B-V}^{\mathrm{h}, V-I}$ & $E_{B-V}^{\mathrm{h}, \mathrm{spec}}$ & $E_{B-V}^{\mathrm{h}, \mathrm{NalD}}$ & $E_{B-V}^{\mathrm{h}}$ \\
\hline $1980 \mathrm{~K}$ & $-0.106 \pm 0.084$ & - & - & - & $0.106 \pm 0.084$ \\
$1986 \mathrm{I}$ & - & - & - & $0.122 \pm 0.090^{4}$ & $0.212 \pm 0.142$ \\
$1988 \mathrm{~A}$ & - & - & - & $0.000 \pm 0.014^{115}$ & $0.090 \pm 0.111$ \\
\hline Notes. Column 1: SN names. Column $2: E_{B-V}^{\mathrm{h}}$ values computed with the C3 method. Column 3: $E_{B-V}^{\mathrm{h}}$ \\
values measured with the $V-I$ colour method. Column 4: $E_{B-V}^{\mathrm{h}}$ computed with the spectrum-fitting \\
technique. Column 5: $E_{B-V}^{\mathrm{h}}$ values estimated from the pEW of the host galaxy Na I D line. Column 6: \\
adopted host galaxy colour excesses. References for the data (superscript numbers) are in Appendix K.
\end{tabular}

Table L4. SN explosion epochs. The full table is available online as supplementary data.

\begin{tabular}{lcccll}
\hline SN & $t_{\ln }(\mathrm{MJD})$ & $t_{\mathrm{fd}}(\mathrm{MJD})$ & $t_{0}$ (MJD) & Phase source & Spectral source \\
\hline $1980 \mathrm{~K}$ & 44494.0 & 44540.0 & $44528.2 \pm 5.9$ & 124, IAUC 3532 & WISeREP \\
1986I & - & 46558.0 & $46556.4 \pm 1.9$ & IAUC 4219 & 4 \\
1988A & 47175.7 & 47176.8 & $47176.2 \pm 0.3$ & IAUC 4533, IAUC 4540 & 125 \\
\hline Notes. Column 1: SN names. Column 2 and 3: SN last non-detection and first detection \\
epochs, respectively. Column 4: explosion epochs estimated with the SNII_ETOS code $( \pm 1 \hat{\sigma}$ \\
error), unless otherwise noted. Column 5: references for $t_{\ln }$ and $t_{\mathrm{fd}}$. Column 6: data source of \\
spectroscopy used to compute $t_{0}$ with the SNII_ETOS code. Reference codes are in Appendix K.
\end{tabular}


Table L5. ${ }^{56} \mathrm{Ni}$ masses and light-curve parameters.

\begin{tabular}{|c|c|c|c|c|c|c|c|c|}
\hline $\mathrm{SN}$ & $x$ & $\begin{array}{l}\text { Range } \\
\text { (d) }\end{array}$ & $N$ & $\begin{array}{l}T_{0} \\
\text { (d) }\end{array}$ & $\begin{array}{c}\log \left(M_{56_{\mathrm{Ni}}}\left[\mathrm{M}_{\odot}\right]\right) \\
(\mathrm{dex})\end{array}$ & $\begin{array}{l}M_{56} \mathrm{Ni} \\
\left(\mathrm{M}_{\odot}\right)\end{array}$ & $\begin{array}{l}M_{V}^{50 \mathrm{~d}} \\
(\mathrm{mag})\end{array}$ & $\rho$ \\
\hline $1980 \mathrm{~K}$ & $\bar{V}$ & $100-285$ & 9 & - & $-1.462 \pm 0.147$ & $0.03655 \pm 0.01273$ & $-17.348 \pm 0.379$ & -0.655 \\
\hline $1986 \mathrm{I}$ & $I$ & $171-213$ & 4 & - & $-1.302 \pm 0.198$ & $0.05535 \pm 0.02661$ & $-16.911 \pm 0.590$ & -0.900 \\
\hline $1988 \mathrm{~A}$ & $R$ & $185-189$ & 3 & - & $-1.109 \pm 0.185$ & $0.08519 \pm 0.03800$ & $-16.488 \pm 0.488$ & -0.930 \\
\hline $1990 \mathrm{E}$ & I & $141-300$ & 7 & - & $-1.383 \pm 0.123$ & $0.04309 \pm 0.01245$ & $-16.702 \pm 0.341$ & -0.899 \\
\hline $1990 \mathrm{~K}$ & $R$ & $100-223$ & 7 & 222 & $-1.470 \pm 0.118$ & $0.03516 \pm 0.00973$ & $-16.705 \pm 0.275$ & -0.810 \\
\hline $1991 \mathrm{G}$ & $I$ & $135-303$ & 7 & - & $-1.778 \pm 0.099$ & $0.01711 \pm 0.00395$ & $-15.365 \pm 0.277$ & -0.798 \\
\hline 1991al & $I$ & $101-129$ & 4 & - & $-1.629 \pm 0.068$ & $0.02379 \pm 0.00375$ & $-15.974 \pm 0.159$ & -0.670 \\
\hline $1992 \mathrm{H}$ & $\mathrm{V}$ & $140-292$ & 7 & - & $-0.788 \pm 0.178$ & $0.17721 \pm 0.07579$ & $-17.670 \pm 0.413$ & -0.899 \\
\hline $1992 \mathrm{ba}$ & $I$ & $150-197$ & 3 & - & $-1.744 \pm 0.126$ & $0.01881 \pm 0.00557$ & $-15.733 \pm 0.305$ & -0.925 \\
\hline $1994 \mathrm{~N}$ & $R$ & $260-290$ & 3 & - & $-2.283 \pm 0.106$ & $0.00537 \pm 0.00133$ & $-14.955 \pm 0.233$ & -0.801 \\
\hline $1995 \mathrm{ad}$ & $I$ & $104-229$ & 7 & 258 & $-1.230 \pm 0.115$ & $0.06099 \pm 0.01644$ & $-17.086 \pm 0.387$ & -0.858 \\
\hline $1996 \mathrm{~W}$ & $I$ & $250-309$ & 3 & - & $-0.952 \pm 0.097$ & $0.11451 \pm 0.02590$ & $-17.469 \pm 0.256$ & -0.823 \\
\hline 1997D & I & $125-176$ & 6 & - & $-2.064 \pm 0.144$ & $0.00912 \pm 0.00311$ & $<-14.436 \pm 0.423$ & -0.824 \\
\hline $1999 \mathrm{ca}$ & $I$ & $203-223$ & 7 & - & $-1.846 \pm 0.070$ & $0.01444 \pm 0.00234$ & $-16.804 \pm 0.167$ & -0.644 \\
\hline $1999 \mathrm{em}$ & $I$ & $132-311$ & 11 & - & $-1.296 \pm 0.062$ & $0.05110 \pm 0.00733$ & $-16.692 \pm 0.138$ & -0.661 \\
\hline 1999ga & $I$ & $121-196$ & 3 & - & $-1.446 \pm 0.120$ & $0.03720 \pm 0.01048$ & $<-16.693 \pm 0.285$ & -0.497 \\
\hline $1999 \mathrm{gi}$ & $I$ & $133-177$ & 7 & 186 & $-1.333 \pm 0.087$ & $0.04739 \pm 0.00959$ & $-16.250 \pm 0.207$ & -0.845 \\
\hline $2001 X$ & $I$ & $123-144$ & 4 & - & $-1.395 \pm 0.097$ & $0.04129 \pm 0.00934$ & $-16.348 \pm 0.231$ & -0.877 \\
\hline $2001 \mathrm{dc}$ & I & $119-153$ & 7 & - & $-2.119 \pm 0.110$ & $0.00785 \pm 0.00202$ & $-15.059 \pm 0.283$ & -0.709 \\
\hline $2002 \mathrm{gw}$ & $I$ & $137-153$ & 3 & - & $-1.631 \pm 0.103$ & $0.02406 \pm 0.00579$ & $-16.017 \pm 0.246$ & -0.869 \\
\hline $2002 \mathrm{hh}$ & $I$ & $167-286$ & 14 & - & $-1.082 \pm 0.069$ & $0.08385 \pm 0.01341$ & $-16.882 \pm 0.285$ & -0.611 \\
\hline $2002 \mathrm{hx}$ & $I$ & $96-160$ & 7 & - & $-1.186 \pm 0.064$ & $0.06587 \pm 0.00976$ & $-16.671 \pm 0.165$ & -0.612 \\
\hline 2003B & $I$ & $223-282$ & 3 & - & $-2.223 \pm 0.115$ & $0.00620 \pm 0.00167$ & $-14.768 \pm 0.271$ & -0.876 \\
\hline $2003 \mathrm{~T}$ & V & $121-137$ & 3 & - & $-1.344 \pm 0.101$ & $0.04653 \pm 0.01097$ & $-16.674 \pm 0.164$ & -0.595 \\
\hline $2003 Z$ & I & $151-208$ & 3 & - & $-2.262 \pm 0.116$ & $0.00567 \pm 0.00154$ & $-14.610 \pm 0.277$ & -0.821 \\
\hline $2003 \mathrm{fb}$ & V & $116-147$ & 5 & - & $-1.482 \pm 0.111$ & $0.03406 \pm 0.00885$ & $-16.016 \pm 0.206$ & -0.725 \\
\hline 2003gd & $I$ & $128-301$ & 17 & 303 & $-1.694 \pm 0.099$ & $0.02076 \pm 0.00480$ & $<-16.405 \pm 0.149$ & -0.407 \\
\hline 2003hd & $I$ & $104-138$ & 4 & - & $-1.362 \pm 0.063$ & $0.04391 \pm 0.00640$ & $-17.056 \pm 0.156$ & -0.629 \\
\hline $2003 \mathrm{hk}$ & $R$ & $117-145$ & 3 & - & $-1.572 \pm 0.105$ & $0.02759 \pm 0.00677$ & $-17.101 \pm 0.211$ & -0.635 \\
\hline $2003 \mathrm{hn}$ & I & $121-151$ & 8 & 187 & $-1.412 \pm 0.056$ & $0.03905 \pm 0.00506$ & $-16.819 \pm 0.136$ & -0.514 \\
\hline 2003ho & $R$ & $102-118$ & 3 & - & $-1.601 \pm 0.105$ & $0.02580 \pm 0.00633$ & $-16.554 \pm 0.267$ & -0.717 \\
\hline 2003iq & $I$ & $120-126$ & 3 & - & $-1.318 \pm 0.082$ & $0.04895 \pm 0.00933$ & $-16.763 \pm 0.200$ & -0.817 \\
\hline $2004 \mathrm{~A}$ & $I$ & $122-241$ & 21 & - & $-1.604 \pm 0.116$ & $0.02579 \pm 0.00701$ & $-15.898 \pm 0.291$ & -0.913 \\
\hline $2004 \mathrm{dj}$ & $I$ & $131-284$ & 17 & - & $-1.902 \pm 0.130$ & $0.01311 \pm 0.00401$ & $-15.846 \pm 0.178$ & -0.320 \\
\hline $2004 \mathrm{eg}$ & $I$ & $150-203$ & 4 & - & $-2.126 \pm 0.188$ & $0.00822 \pm 0.00373$ & $<-15.077 \pm 0.551$ & -0.744 \\
\hline $2004 \mathrm{ej}$ & $V$ & $120-194$ & 14 & - & $-1.793 \pm 0.166$ & $0.01733 \pm 0.00687$ & $-16.494 \pm 0.380$ & -0.881 \\
\hline 2004 et & $I$ & $135-319$ & 23 & 372 & $-1.037 \pm 0.071$ & $0.09307 \pm 0.01532$ & $-17.645 \pm 0.213$ & -0.759 \\
\hline $2004 f x$ & $V$ & $109-161$ & 24 & - & $-1.802 \pm 0.173$ & $0.01708 \pm 0.00708$ & $-15.720 \pm 0.400$ & -0.906 \\
\hline 2005af & $V$ & $123-162$ & 20 & 234 & $-1.477 \pm 0.179$ & $0.03630 \pm 0.01562$ & $<-15.212 \pm 0.383$ & -0.836 \\
\hline $2005 \mathrm{au}$ & $I$ & $102-137$ & 5 & - & $-1.195 \pm 0.085$ & $0.06506 \pm 0.01286$ & $-17.291 \pm 0.273$ & -0.718 \\
\hline 2005ay & $R$ & $117-236$ & 5 & - & $-1.778 \pm 0.109$ & $0.01721 \pm 0.00439$ & $-15.512 \pm 0.239$ & -0.828 \\
\hline $2005 \mathrm{cs}$ & $I$ & $150-311$ & 18 & - & $-2.241 \pm 0.076$ & $0.00583 \pm 0.00103$ & $-15.371 \pm 0.133$ & -0.474 \\
\hline $2005 \mathrm{dx}$ & V & $103-120$ & 3 & - & $-2.064 \pm 0.194$ & $0.00954 \pm 0.00448$ & $-15.583 \pm 0.445$ & -0.878 \\
\hline $2006 \mathrm{my}$ & $I$ & $177-261$ & 8 & - & $-1.674 \pm 0.141$ & $0.02233 \pm 0.00745$ & $<-15.361 \pm 0.473$ & -0.768 \\
\hline 2006 ov & $I$ & $145-231$ & 9 & - & $-2.051 \pm 0.267$ & $0.01074 \pm 0.00728$ & $<-16.568 \pm 0.707$ & -0.961 \\
\hline 2007 aa & $i$ & $106-121$ & 7 & - & $-1.522 \pm 0.143$ & $0.03174 \pm 0.01074$ & $-16.556 \pm 0.407$ & -0.907 \\
\hline $2007 \mathrm{hv}$ & $i$ & $113-131$ & 6 & - & $-1.326 \pm 0.125$ & $0.04920 \pm 0.01446$ & $-16.698 \pm 0.400$ & -0.887 \\
\hline $2007 \mathrm{it}$ & $I$ & $137-312$ & 7 & - & $-0.987 \pm 0.130$ & $0.10776 \pm 0.03299$ & $-17.400 \pm 0.417$ & -0.899 \\
\hline 2007od & $I$ & $239-316$ & 3 & - & $>-2.482 \pm 0.148$ & $>0.00349 \pm 0.00123$ & $-17.438 \pm 0.332$ & -0.713 \\
\hline $2008 \mathrm{~K}$ & $V$ & $122-182$ & 4 & - & $-1.611 \pm 0.180$ & $0.02669 \pm 0.01155$ & $-16.806 \pm 0.378$ & -0.713 \\
\hline $2008 \mathrm{M}$ & $V$ & $96-120$ & 5 & - & $-1.605 \pm 0.172$ & $0.02686 \pm 0.01107$ & $-16.504 \pm 0.396$ & -0.900 \\
\hline 2008aw & $V$ & $103-133$ & 5 & 117 & $-1.074 \pm 0.114$ & $0.08729 \pm 0.02331$ & $-17.228 \pm 0.230$ & -0.700 \\
\hline $2008 \mathrm{bk}$ & $I$ & $175-292$ & 5 & - & $-2.064 \pm 0.094$ & $0.00883 \pm 0.00193$ & $-14.992 \pm 0.366$ & -0.806 \\
\hline $2008 \mathrm{gz}$ & $I$ & $127-273$ & 26 & - & $-1.253 \pm 0.081$ & $0.05683 \pm 0.01069$ & $<-16.366 \pm 0.194$ & -0.784 \\
\hline 2008in & $I$ & $113-152$ & 9 & - & $-1.640 \pm 0.258$ & $0.02733 \pm 0.01778$ & $-16.134 \pm 0.644$ & -0.979 \\
\hline $2009 \mathrm{~N}$ & $I$ & $109-191$ & 23 & - & $-1.891 \pm 0.161$ & $0.01377 \pm 0.00528$ & $-15.357 \pm 0.404$ & -0.951 \\
\hline 2009at & $I$ & $115-142$ & 3 & - & $-1.750 \pm 0.115$ & $0.01842 \pm 0.00496$ & $-16.153 \pm 0.332$ & -0.841 \\
\hline 2009ay & $R$ & $100-178$ & 8 & - & $-0.932 \pm 0.116$ & $0.12120 \pm 0.03296$ & $-17.720 \pm 0.309$ & -0.790 \\
\hline 2009 bw & $I$ & $138-235$ & 10 & 236 & $-1.737 \pm 0.120$ & $0.01904 \pm 0.00536$ & $-16.393 \pm 0.316$ & -0.897 \\
\hline 2009 dd & $R$ & $139-298$ & 6 & - & $-1.474 \pm 0.112$ & $0.03471 \pm 0.00910$ & $-16.385 \pm 0.259$ & -0.716 \\
\hline $2009 \mathrm{hd}$ & $I$ & $158-271$ & 5 & 220 & $-1.959 \pm 0.066$ & $0.01112 \pm 0.00170$ & $-16.632 \pm 0.188$ & -0.677 \\
\hline $2009 \mathrm{ib}$ & $R$ & $149-262$ & 4 & - & $-1.356 \pm 0.075$ & $0.04472 \pm 0.00778$ & $-15.790 \pm 0.131$ & -0.524 \\
\hline 2009 md & $I$ & $123-180$ & 4 & - & $-2.097 \pm 0.078$ & $0.00813 \pm 0.00147$ & $-15.365 \pm 0.195$ & -0.689 \\
\hline $2010 \mathrm{aj}$ & $I$ & $95-107$ & 4 & - & $-2.088 \pm 0.075$ & $0.00829 \pm 0.00144$ & $-16.764 \pm 0.203$ & -0.690 \\
\hline PTF10gva & $r$ & $175-213$ & 3 & - & $-1.111 \pm 0.138$ & $0.08146 \pm 0.02655$ & - & - \\
\hline $2011 \mathrm{fd}$ & $I$ & $126-260$ & 12 & 292 & $-1.498 \pm 0.125$ & $0.03311 \pm 0.00973$ & - & - \\
\hline PTF11go & $R$ & $97-160$ & 15 & - & $-1.593 \pm 0.160$ & $0.02732 \pm 0.01042$ & - & - \\
\hline PTF11htj & $r$ & $133-173$ & 6 & - & $-1.309 \pm 0.175$ & $0.05324 \pm 0.02236$ & - & - \\
\hline PTF11izt & $R$ & $111-182$ & 49 & - & $-1.651 \pm 0.159$ & $0.02388 \pm 0.00905$ & - & - \\
\hline 2012A & $I$ & $125-310$ & 9 & - & $-1.766 \pm 0.111$ & $0.01771 \pm 0.00460$ & $-16.454 \pm 0.284$ & -0.888 \\
\hline 2012aw & $I$ & $221-309$ & 22 & 537 & $-1.269 \pm 0.055$ & $0.05426 \pm 0.00690$ & $-16.847 \pm 0.135$ & -0.602 \\
\hline $2012 \mathrm{br}$ & $R$ & $99-263$ & 37 & - & $-1.219 \pm 0.254$ & $0.07166 \pm 0.04576$ & - & - \\
\hline $2012 \mathrm{~cd}$ & $r$ & $108-216$ & 6 & 228 & $-1.038 \pm 0.168$ & $0.09874 \pm 0.03967$ & - & - \\
\hline $2012 \mathrm{ec}$ & $I$ & $111-163$ & 8 & - & $-1.545 \pm 0.115$ & $0.02953 \pm 0.00796$ & $-16.336 \pm 0.290$ & -0.891 \\
\hline PTF12grj & $r$ & $107-139$ & 6 & - & $-1.565 \pm 0.169$ & $0.02937 \pm 0.01187$ & - & - \\
\hline PTF12hsx & $R$ & $135-223$ & 575 & - & $-1.090 \pm 0.225$ & $0.09296 \pm 0.05158$ & - & - \\
\hline $2013 \mathrm{~K}$ & $R$ & $211-303$ & 5 & - & $-1.593 \pm 0.123$ & $0.02657 \pm 0.00768$ & $-16.711 \pm 0.297$ & -0.851 \\
\hline 2013ab & $I$ & $107-218$ & 19 & 312 & $-1.482 \pm 0.095$ & $0.03376 \pm 0.00747$ & $-16.199 \pm 0.234$ & -0.867 \\
\hline 2013am & $I$ & $230-314$ & 5 & - & $-1.695 \pm 0.163$ & $0.02166 \pm 0.00842$ & $-15.470 \pm 0.358$ & -0.743 \\
\hline 2013bu & $I$ & $130-281$ & 9 & - & $-1.888 \pm 0.093$ & $0.01324 \pm 0.00287$ & $-16.104 \pm 0.217$ & -0.604 \\
\hline
\end{tabular}


Table L5 - continued

\begin{tabular}{|c|c|c|c|c|c|c|c|c|}
\hline $\mathrm{SN}$ & $x$ & $\begin{array}{l}\text { Range } \\
\text { (d) }\end{array}$ & $N$ & $\begin{array}{l}T_{0} \\
\text { (d) }\end{array}$ & $\begin{array}{c}\log \left(M_{56_{\mathrm{Ni}}}\left[\mathrm{M}_{\odot}\right]\right) \\
(\mathrm{dex})\end{array}$ & $\begin{array}{c}M_{56} \mathrm{Ni} \\
\left(\mathrm{M}_{\odot}\right) \\
\end{array}$ & $\begin{array}{l}M_{V}^{50 \mathrm{~d}} \\
(\mathrm{mag}) \\
\end{array}$ & $\rho$ \\
\hline 2013by & $i$ & $104-153$ & 10 & 176 & $-1.512 \pm 0.151$ & $0.03268 \pm 0.01171$ & $-17.397 \pm 0.436$ & -0.902 \\
\hline 2013ej & $I$ & $107-226$ & 107 & 172 & $-1.551 \pm 0.060$ & $0.02839 \pm 0.00394$ & $-16.950 \pm 0.149$ & -0.675 \\
\hline $2013 \mathrm{fs}$ & $r$ & $102-107$ & 4 & - & $-1.323 \pm 0.106$ & $0.04897 \pm 0.01213$ & $-16.739 \pm 0.213$ & -0.762 \\
\hline 2013hj & $I$ & $117-168$ & 19 & - & $-1.092 \pm 0.073$ & $0.08206 \pm 0.01389$ & $-17.564 \pm 0.178$ & -0.767 \\
\hline iPTF13dkz & $r$ & $152-174$ & 5 & - & $-1.173 \pm 0.205$ & $0.07506 \pm 0.03750$ & - & - \\
\hline LSQ13dpa & $i$ & $144-152$ & 3 & - & $-1.101 \pm 0.135$ & $0.08317 \pm 0.02649$ & $-17.181 \pm 0.470$ & -0.913 \\
\hline $2014 \mathrm{G}$ & $I$ & $95-165$ & 30 & 127 & $-1.146 \pm 0.077$ & $0.07258 \pm 0.01297$ & $-17.203 \pm 0.199$ & -0.793 \\
\hline $2014 \mathrm{cx}$ & $I$ & $120-150$ & 10 & - & $-1.251 \pm 0.103$ & $0.05771 \pm 0.01388$ & $-16.668 \pm 0.263$ & -0.884 \\
\hline 2014cy & $R$ & $128-164$ & 4 & 113 & $-1.820 \pm 0.117$ & $0.01569 \pm 0.00431$ & $-15.584 \pm 0.269$ & -0.861 \\
\hline $2014 d w$ & $r$ & $97-143$ & 18 & 85 & $-1.544 \pm 0.158$ & $0.03053 \pm 0.01149$ & $-16.238 \pm 0.487$ & -0.734 \\
\hline ASASSN-14dq & $I$ & $108-204$ & 10 & 266 & $-1.308 \pm 0.101$ & $0.05055 \pm 0.01192$ & $-16.967 \pm 0.249$ & -0.826 \\
\hline ASASSN-14ha & $i$ & $140-181$ & 18 & - & $-2.030 \pm 0.120$ & $0.00970 \pm 0.00273$ & $-15.963 \pm 0.369$ & -0.859 \\
\hline OGLE14-18 & $I$ & $182-235$ & 6 & - & $-1.367 \pm 0.123$ & $0.04471 \pm 0.01292$ & - & - \\
\hline $2015 \mathrm{~V}$ & $I$ & $124-217$ & 33 & - & $-1.643 \pm 0.107$ & $0.02345 \pm 0.00587$ & $-15.751 \pm 0.287$ & -0.871 \\
\hline $2015 \mathrm{~W}$ & $i$ & $253-289$ & 4 & - & $-1.372 \pm 0.110$ & $0.04385 \pm 0.01129$ & $-17.129 \pm 0.314$ & -0.714 \\
\hline 2015an & $i$ & $148-218$ & 13 & - & $-1.575 \pm 0.113$ & $0.02752 \pm 0.00728$ & $-17.184 \pm 0.363$ & -0.873 \\
\hline $2015 \mathrm{ba}$ & $i$ & $166-259$ & 3 & - & $-1.840 \pm 0.114$ & $0.01496 \pm 0.00400$ & $-17.020 \pm 0.221$ & -0.613 \\
\hline $2015 \mathrm{cz}$ & $I$ & $125-143$ & 4 & - & $-1.344 \pm 0.093$ & $0.04634 \pm 0.01004$ & $-17.158 \pm 0.272$ & -0.750 \\
\hline ASASSN-15oz & $i$ & $185-261$ & 18 & 126 & $-0.875 \pm 0.124$ & $0.13890 \pm 0.04048$ & $-17.682 \pm 0.316$ & -0.887 \\
\hline $2016 \mathrm{X}$ & $I$ & $98-196$ & 37 & 205 & $-1.349 \pm 0.147$ & $0.04741 \pm 0.01652$ & $-16.856 \pm 0.371$ & -0.937 \\
\hline 2016aqf & $i$ & $154-307$ & 11 & 332 & $-1.670 \pm 0.137$ & $0.02247 \pm 0.00727$ & $-16.024 \pm 0.394$ & -0.902 \\
\hline $2016 \mathrm{bkv}$ & $I$ & $202-307$ & 29 & - & $-1.817 \pm 0.106$ & $0.01570 \pm 0.00389$ & $-14.537 \pm 0.300$ & -0.800 \\
\hline 2016gfy & $I$ & $122-238$ & 11 & 307 & $-1.122 \pm 0.103$ & $0.07766 \pm 0.01868$ & $-17.145 \pm 0.263$ & -0.883 \\
\hline 2016ija & $i$ & $96-105$ & 6 & - & $-1.582 \pm 0.144$ & $0.02766 \pm 0.00943$ & $-16.691 \pm 0.490$ & -0.916 \\
\hline 2017it & $I$ & $118-135$ & 18 & - & $-1.257 \pm 0.060$ & $0.05587 \pm 0.00776$ & $-16.817 \pm 0.149$ & -0.626 \\
\hline 2017ahn & $i$ & $101-142$ & 5 & 98 & $-1.316 \pm 0.132$ & $0.05059 \pm 0.01574$ & $-16.751 \pm 0.464$ & -0.914 \\
\hline 2017eaw & $I$ & $128-313$ & 136 & - & $-1.087 \pm 0.069$ & $0.08289 \pm 0.01325$ & $-17.265 \pm 0.203$ & -0.752 \\
\hline $2017 \mathrm{gmr}$ & $I$ & $103-146$ & 6 & - & $-0.857 \pm 0.093$ & $0.14222 \pm 0.03081$ & $-17.655 \pm 0.235$ & -0.852 \\
\hline 2018 cuf & $i$ & $123-273$ & 18 & - & $-1.417 \pm 0.121$ & $0.03980 \pm 0.01131$ & $-16.898 \pm 0.359$ & -0.877 \\
\hline 2018hwm & $r$ & $171-191$ & 3 & - & $-2.205 \pm 0.124$ & $0.00650 \pm 0.00189$ & $-15.041 \pm 0.284$ & -0.822 \\
\hline
\end{tabular}

Table L6. $M_{R}^{\max }$ value for the LOSS set and our SN sample.

\begin{tabular}{|c|c|c|c|c|c|c|c|c|c|}
\hline $\mathrm{SN}^{\dagger}$ & $\begin{array}{l}M_{R}^{\max } \\
(\operatorname{mag})\end{array}$ & $\mathrm{SN}^{\ddagger}$ & $\begin{array}{l}M_{R}^{\max } \\
(\mathrm{mag})\end{array}$ & $\mathrm{SN}^{\ddagger}$ & $\begin{array}{l}M_{R}^{\max } \\
(\mathrm{mag})\end{array}$ & $\mathrm{SN}^{\ddagger}$ & $\begin{array}{l}M_{R}^{\max } \\
(\operatorname{mag})\end{array}$ & $\mathrm{SN}^{\ddagger}$ & $\begin{array}{l}M_{R}^{\max } \\
\text { (mag) }\end{array}$ \\
\hline 1999an & -16.931 & $1980 \mathrm{~K}$ & -18.764 & $2003 \mathrm{hk}$ & -18.303 & 2009ay & -18.552 & LSQ13dpa & -17.694 \\
\hline $1999 \mathrm{bg}$ & -16.473 & 1986I & -16.730 & 2003hn & -17.602 & 2009bw & -17.309 & $2014 \mathrm{G}$ & -18.411 \\
\hline $1999 \mathrm{br}$ & -15.526 & $1988 \mathrm{~A}$ & -16.639 & 2003ho & -17.415 & 2009dd & -16.993 & $2014 c x$ & -16.957 \\
\hline $1999 \mathrm{em}$ & -17.192 & $1990 \mathrm{E}$ & -17.401 & 2003iq & -17.347 & 2009hd & -17.327 & 2014 cy & -16.122 \\
\hline 1999gi & -16.728 & $1990 \mathrm{~K}$ & -17.784 & $2004 \mathrm{~A}$ & -16.159 & $2009 \mathrm{ib}$ & -16.266 & $2014 d w$ & -17.312 \\
\hline $2000 \mathrm{dc}$ & -17.921 & $1991 \mathrm{G}$ & -15.729 & $2004 \mathrm{dj}$ & -16.232 & $2009 \mathrm{md}$ & -15.779 & ASASSN-14dq & -17.653 \\
\hline 2000el & -16.312 & 1991al & -16.836 & $2004 \mathrm{eg}$ & -15.693 & $2010 \mathrm{aj}$ & -17.836 & ASASSN-14ha & -16.379 \\
\hline $2001 \mathrm{bq}$ & -17.577 & $1992 \mathrm{H}$ & -18.067 & $2004 \mathrm{ej}$ & -17.026 & PTF10gva & -18.794 & OGLE14-18 & -17.103 \\
\hline $2001 \mathrm{dc}$ & -15.283 & $1992 \mathrm{ba}$ & -16.218 & 2004 et & -17.974 & $2011 \mathrm{fd}$ & -16.998 & $2015 \mathrm{~V}$ & -15.970 \\
\hline $2001 f z$ & -15.865 & $1994 \mathrm{~N}$ & -15.371 & $2004 f x$ & -15.984 & PTF11go & -16.743 & $2015 \mathrm{~W}$ & -17.893 \\
\hline $2002 b x$ & -16.901 & $1995 \mathrm{ad}$ & -17.782 & 2005 af & -17.413 & PTF11htj & -16.946 & 2015an & -17.697 \\
\hline 2002ce & -15.319 & 1996W & -17.798 & $2005 \mathrm{au}$ & -17.974 & PTF11izt & -16.348 & 2015ba & -17.750 \\
\hline $2002 d q$ & -16.232 & 1997D & -14.986 & 2005ay & -15.912 & $2012 \mathrm{~A}$ & -17.176 & $2015 \mathrm{cz}$ & -17.627 \\
\hline $2002 \mathrm{ds}$ & -17.197 & 1999ca & -18.086 & $2005 \mathrm{cs}$ & -15.700 & 2012aw & -17.181 & ASASSN-15oz & -18.478 \\
\hline $2002 \mathrm{gd}$ & -15.939 & $1999 \mathrm{em}$ & -17.192 & $2005 d x$ & -16.381 & $2012 \mathrm{br}$ & -17.764 & $2016 X$ & -17.588 \\
\hline $2002 \mathrm{hh}$ & -17.107 & 1999ga & -17.320 & 2006my & -16.896 & $2012 \mathrm{~cd}$ & -18.680 & 2016aqf & -16.136 \\
\hline $2003 Z$ & -14.958 & 1999gi & -16.728 & 2006 ov & -16.195 & $2012 \mathrm{ec}$ & -16.695 & 2016bkv & -15.741 \\
\hline 2003 ao & -16.339 & $2001 X$ & -16.726 & 2007aa & -16.703 & PTF12grj & -16.963 & 2016gfy & -17.599 \\
\hline 2003hl & -17.364 & $2001 d c$ & -15.283 & $2007 \mathrm{hv}$ & -17.158 & PTF12hsx & -17.331 & 2016ija & -17.238 \\
\hline 2003iq & -17.347 & 2002gw & -16.310 & 2007it & -18.234 & $2013 \mathrm{~K}$ & -16.430 & 2017it & -17.178 \\
\hline 2004 et & -17.974 & $2002 \mathrm{hh}$ & -17.107 & $2008 \mathrm{~K}$ & -17.869 & $2013 a b$ & -16.804 & 2017ahn & -18.286 \\
\hline $2004 \mathrm{fc}$ & -16.642 & $2002 h x$ & -17.596 & $2008 \mathrm{M}$ & -17.236 & 2013am & -15.958 & 2017eaw & -17.828 \\
\hline $2004 f x$ & -15.984 & 2003B & -15.248 & 2008aw & -18.181 & 2013bu & -16.947 & $2017 \mathrm{gmr}$ & -18.005 \\
\hline $2005 \mathrm{ad}$ & -15.882 & $2003 \mathrm{~T}$ & -17.131 & 2008bk & -15.245 & 2013by & -18.411 & 2018cuf & -17.224 \\
\hline 2005ay & -15.912 & $2003 Z$ & -14.958 & $2008 \mathrm{gz}$ & -17.679 & 2013ej & -17.900 & 2018hwm & -15.191 \\
\hline 2006be & -16.972 & $2003 \mathrm{fb}$ & -16.582 & 2008in & -16.596 & $2013 \mathrm{fs}$ & -17.623 & - & - \\
\hline 2006bp & -17.622 & 2003gd & -16.859 & $2009 \mathrm{~N}$ & -15.609 & 2013hj & -18.270 & - & - \\
\hline $2006 \mathrm{ca}$ & -17.202 & 2003hd & -17.635 & 2009at & -17.283 & iPTF13dkz & -16.470 & - & - \\
\hline
\end{tabular}

${ }^{\dagger}$ LOSS set. Original $M_{R}^{\max }$ values (listed in Li et al. 2011) were recalibrated using $E_{B-V}^{G}$ values from Schlafly \& Finkbeiner (2011), $\mu$ estimates listed in Column 8 of Table L2, and $E_{B-V}^{\mathrm{h}}$ values reported in Column 6 of Table L3.

$\ddagger$ Our SN sample. 
Table L7. Steepness parameters. The full table is available online as supplementary data.

\begin{tabular}{|c|c|c|c|c|c|c|c|c|c|c|c|c|}
\hline SN & $S_{g}$ & $S_{V}$ & $S_{r}$ & $S_{R}$ & $S_{i}$ & $S_{I}$ & $S_{q}^{*}$ & $S_{V}^{*}$ & $S_{r}^{*}$ & $S_{R}^{*}$ & $S_{i}^{*}$ & $S_{I}^{*}$ \\
\hline $1980 \mathrm{~K}$ & - & $0.159(39)$ & - & - & - & - & $0.149(49)$ & - & $0.118(42)$ & $0.134(40)$ & $0.131(44)$ & $0.125(37)$ \\
\hline $1991 \mathrm{G}$ & - & $0.120(46)$ & - & $0.089(18)$ & - & $0.069(20)$ & $0.109(14)$ & $0.104(23)$ & $0.080(14)$ & $0.083(26)$ & $0.086(24)$ & $0.090(22)$ \\
\hline $1992 \mathrm{ba}$ & - & $0.098(23)$ & - & - & - & $0.082(25)$ & $0.102(21)$ & $0.108(40)$ & $0.076(19)$ & $0.084(23)$ & $0.081(26)$ & $0.079(28)$ \\
\hline
\end{tabular}

Notes. Steepness values are in mag d ${ }^{-1}$ units. Numbers in parentheses are $1 \sigma$ errors in units of the last significant digits.

*Values estimated from the steepnesses in other bands (see Section 4.5).

Table L8. $S_{b}$ to $S_{x}$ transformation parameters.

\begin{tabular}{cccccccccccc}
\hline$x$ & $b$ & $\begin{array}{c}c_{x, b} \\
\left(\mathrm{mag} \mathrm{d}^{-1}\right)\end{array}$ & $d_{x, b}$ & $\begin{array}{c}\hat{\sigma}_{x, b} \\
\left(\mathrm{mag} \mathrm{d}^{-1}\right)\end{array}$ & $N$ & $x$ & $b$ & $\begin{array}{c}c_{x, b} \\
\left(\mathrm{mag} \mathrm{d}^{-1}\right)\end{array}$ & $d_{x, b}$ & $\begin{array}{c}\hat{\sigma}_{x, b} \\
\left(\mathrm{mag} \mathrm{d}^{-1}\right)\end{array}$ \\
\hline$g$ & $V$ & $-0.008 \pm 0.027$ & $0.986 \pm 0.208$ & 0.030 & 15 & $V$ & $g$ & $0.015 \pm 0.033$ & $0.969 \pm 0.272$ & 0.030 & 15 \\
$g$ & $r$ & $0.004 \pm 0.015$ & $1.161 \pm 0.145$ & 0.021 & 15 & $V$ & $r$ & $0.026 \pm 0.018$ & $1.129 \pm 0.157$ & 0.031 \\
$g$ & $R$ & $0.042 \pm 0.006$ & $0.817 \pm 0.056$ & 0.012 & 8 & $V$ & $R$ & $0.017 \pm 0.008$ & $1.072 \pm 0.075$ & 0.024 & 29 \\
$g$ & $i$ & $-0.000 \pm 0.025$ & $1.118 \pm 0.227$ & 0.030 & 15 & $V$ & $i$ & $0.016 \pm 0.010$ & $1.099 \pm 0.045$ & 0.033 & 19 \\
$g$ & $I$ & $0.054 \pm 0.016$ & $0.677 \pm 0.089$ & 0.020 & 8 & $V$ & $I$ & $0.011 \pm 0.011$ & $1.187 \pm 0.093$ & 0.027 & 28 \\
$r$ & $g$ & $-0.001 \pm 0.008$ & $0.842 \pm 0.017$ & 0.018 & 15 & $R$ & $g$ & $-0.041 \pm 0.010$ & $1.146 \pm 0.063$ & 0.014 & 8 \\
$r$ & $V$ & $-0.014 \pm 0.023$ & $0.832 \pm 0.162$ & 0.027 & 18 & $R$ & $V$ & $-0.002 \pm 0.010$ & $0.853 \pm 0.077$ & 0.022 & 29 \\
$r$ & $R$ & $0.026 \pm 0.011$ & $0.686 \pm 0.114$ & 0.015 & 10 & $R$ & $r$ & $-0.015 \pm 0.020$ & $1.256 \pm 0.198$ & 0.021 & 10 \\
$r$ & $i$ & $-0.001 \pm 0.020$ & $0.939 \pm 0.184$ & 0.031 & 17 & $R$ & $i$ & $0.039 \pm 0.024$ & $0.680 \pm 0.123$ & 0.037 & 11 \\
$r$ & $I$ & $0.022 \pm 0.011$ & $0.717 \pm 0.081$ & 0.015 & 10 & $R$ & $I$ & $0.002 \pm 0.009$ & $1.053 \pm 0.067$ & 0.025 & 28 \\
$i$ & $g$ & $0.007 \pm 0.024$ & $0.853 \pm 0.201$ & 0.027 & 15 & $I$ & $g$ & $-0.040 \pm 0.035$ & $1.189 \pm 0.199$ & 0.027 & 8 \\
$i$ & $V$ & $-0.004 \pm 0.009$ & $0.848 \pm 0.051$ & 0.029 & 19 & $I$ & $V$ & $0.005 \pm 0.010$ & $0.755 \pm 0.072$ & 0.022 & 28 \\
$i$ & $r$ & $0.011 \pm 0.010$ & $0.986 \pm 0.029$ & 0.032 & 17 & $I$ & $r$ & $-0.009 \pm 0.017$ & $1.203 \pm 0.145$ & 0.020 & 10 \\
$i$ & $R$ & $0.022 \pm 0.029$ & $0.872 \pm 0.291$ & 0.042 & 11 & $I$ & $R$ & $0.012 \pm 0.008$ & $0.855 \pm 0.055$ & 0.022 & 28 \\
$i$ & $I$ & $0.000 \pm 0.016$ & $1.037 \pm 0.109$ & 0.028 & 11 & $I$ & $i$ & $0.024 \pm 0.015$ & $0.792 \pm 0.084$ & 0.024 & 11 \\
\hline
\end{tabular}

Table L9. Absolute magnitudes at $50 \mathrm{~d}$ since explosion. The full table is available online as supplementary data.

\begin{tabular}{lccccc}
\hline SN & $M_{g}^{50 \mathrm{~d}}$ & $M_{r}^{50 \mathrm{~d}}$ & $M_{R}^{50 \mathrm{~d}}$ & $M_{i}^{50 \mathrm{~d}}$ & $M_{I}^{50 \mathrm{~d}}$ \\
\hline $1980 \mathrm{~K}$ & $-17.099 \pm 0.429^{*}$ & $>-18.255 \pm 0.270^{*}$ & $>-18.385 \pm 0.253$ & $>-17.897 \pm 0.219^{*}$ & $>-18.287 \pm 0.194$ \\
$1986 \mathrm{I}$ & - & $-16.490 \pm 0.548^{*}$ & $-16.619 \pm 0.532$ & $-16.739 \pm 0.489^{*}$ & $-17.129 \pm 0.471$ \\
$1990 \mathrm{E}$ & - & $-17.084 \pm 0.324^{*}$ & $-17.214 \pm 0.314$ & $-16.915 \pm 0.302^{*}$ & $-17.304 \pm 0.289$ \\
\hline
\end{tabular}

${ }^{*}$ Values estimated using photometry in other bands (see Section 4.6 ).

Table L10. SZB sample.

\begin{tabular}{|c|c|c|c|c|c|c|c|c|c|}
\hline IAU SN & $\begin{array}{l}E_{B-V}^{G} \\
(\mathrm{mag})\end{array}$ & $\begin{array}{c}c z_{\text {helio }}^{\mathrm{SN}} \\
\left(\mathrm{km} \mathrm{s}^{-1}\right)\end{array}$ & $\begin{array}{c}\mu \\
(\mathrm{mag})\end{array}$ & $\begin{array}{c}t_{0} \\
(\mathrm{MJD})\end{array}$ & $\begin{array}{l}M_{R}^{\max } \\
(\mathrm{mag})\end{array}$ & $\begin{array}{c}M_{r_{\mathrm{ZTF}}^{50 \mathrm{~d}}}^{\text {(mag) }} \\
\end{array}$ & $\begin{array}{c}S_{r_{\mathrm{ZTF}}} \\
\left(\mathrm{mag} \mathrm{d}^{-1}\right)\end{array}$ & $\begin{array}{c}\log M_{56 \mathrm{Ni}}^{\mathrm{NMS}} \\
(\mathrm{dex})\end{array}$ & $\begin{array}{c}\log M_{56 \mathrm{Ni}}^{\text {tail }} \\
(\mathrm{dex})\end{array}$ \\
\hline $2018 \mathrm{ccb}$ & 0.139 & 4740 & $33.90 \pm 0.12$ & $58267.9 \pm 1.4$ & -17.313 & $-16.67 \pm 0.39$ & $0.116 \pm 0.009$ & $-1.505 \pm 0.145$ & $-1.399 \pm 0.162$ \\
\hline $2018 \mathrm{fpx}$ & 0.210 & 7500 & $35.03 \pm 0.09$ & $58356.6 \pm 4.0$ & $<-18.246$ & $-17.75 \pm 0.39$ & $0.059 \pm 0.011$ & $-1.033 \pm 0.153$ & $-0.971 \pm 0.161$ \\
\hline 2018 hcp & 0.030 & 5934 & $34.59 \pm 0.11$ & $58377.0 \pm 1.5$ & -17.381 & $-16.94 \pm 0.39$ & $0.055 \pm 0.004$ & $-1.218 \pm 0.144$ & $-1.215 \pm 0.160$ \\
\hline 2019va & 0.016 & 2637 & $33.03 \pm 0.19$ & $58497.6 \pm 3.1$ & -17.488 & $-17.20 \pm 0.41$ & $0.065 \pm 0.004$ & $-1.205 \pm 0.148$ & $-0.962 \pm 0.170$ \\
\hline $2019 v \mathrm{v}$ & 0.011 & 6039 & $34.60 \pm 0.12$ & $58500.6 \pm 1.0$ & -18.469 & $-17.88 \pm 0.39$ & $0.074 \pm 0.011$ & $-1.069 \pm 0.149$ & $-0.872 \pm 0.160$ \\
\hline 2019clp & 0.031 & 7127 & $35.01 \pm 0.11$ & $58569.0 \pm 0.5$ & -18.490 & $-17.66 \pm 0.39$ & $0.068 \pm 0.009$ & $-1.103 \pm 0.147$ & $-1.074 \pm 0.158$ \\
\hline $2019 \mathrm{cvz}$ & 0.011 & 5578 & $34.39 \pm 0.13$ & $58577.6 \pm 1.0$ & -17.282 & $-17.09 \pm 0.39$ & $0.076 \pm 0.008$ & $-1.274 \pm 0.146$ & $-1.177 \pm 0.162$ \\
\hline 2019dma & 0.113 & 4880 & $33.89 \pm 0.15$ & $58560.2 \pm 6.4$ & $<-17.395$ & $-17.22 \pm 0.41$ & $0.143 \pm 0.017$ & $-1.426 \pm 0.150$ & $-1.585 \pm 0.167$ \\
\hline 2019 etp & 0.044 & 4683 & $33.77 \pm 0.16$ & $58607.2 \pm 2.7$ & $<-17.788$ & $-17.45 \pm 0.40$ & $0.048 \pm 0.004$ & $-1.054 \pm 0.147$ & $-1.065 \pm 0.165$ \\
\hline 2019ffn & 0.102 & 7800 & $35.09 \pm 0.11$ & $58616.9 \pm 0.4$ & -18.300 & $-17.81 \pm 0.39$ & $0.071 \pm 0.003$ & $-1.078 \pm 0.143$ & $-1.039 \pm 0.159$ \\
\hline $2019 \mathrm{gqk}$ & 0.079 & 3900 & $33.35 \pm 0.19$ & $58625.6 \pm 4.9$ & $<-16.894$ & $-16.69 \pm 0.42$ & $0.056 \pm 0.004$ & $-1.288 \pm 0.149$ & $-1.247 \pm 0.171$ \\
\hline $2019 \mathrm{hhh}$ & 0.281 & 4500 & $33.72 \pm 0.17$ & $58635.4 \pm 4.5$ & -17.059 & $-16.69 \pm 0.42$ & $0.081 \pm 0.004$ & $-1.395 \pm 0.149$ & $-1.254 \pm 0.172$ \\
\hline $2019 \mathrm{hkj}$ & 0.071 & 5400 & $34.17 \pm 0.15$ & $58620.6 \pm 9.1$ & $<-17.051$ & $-16.87 \pm 0.40$ & $0.103 \pm 0.008$ & $-1.421 \pm 0.146$ & $-1.332 \pm 0.167$ \\
\hline 2019iex & 0.063 & 4211 & $33.54 \pm 0.19$ & $58658.9 \pm 1.5$ & -17.503 & $-17.21 \pm 0.41$ & $0.173 \pm 0.012$ & $-1.486 \pm 0.149$ & $-1.339 \pm 0.171$ \\
\hline 2019jyw & 0.029 & 3489 & $33.16 \pm 0.21$ & $58659.5 \pm 2.9$ & -17.850 & $-17.03 \pm 0.43$ & $0.070 \pm 0.004$ & $-1.269 \pm 0.150$ & $-1.140 \pm 0.174$ \\
\hline $2019 \mathrm{lkx}$ & 0.454 & 3000 & $32.81 \pm 0.23$ & $58677.0 \pm 1.5$ & -17.220 & $-16.85 \pm 0.47$ & $0.052 \pm 0.002$ & $-1.229 \pm 0.157$ & $-1.224 \pm 0.190$ \\
\hline 2019vus & 0.025 & 7500 & $35.08 \pm 0.09$ & $58806.6 \pm 1.0$ & $<-17.374$ & $-16.94 \pm 0.38$ & $0.057 \pm 0.009$ & $-1.224 \pm 0.148$ & $-1.174 \pm 0.163$ \\
\hline $2020 \mathrm{ckb}$ & 0.075 & 7503 & $34.98 \pm 0.11$ & $58861.9 \pm 4.5$ & $<-17.867$ & $-17.50 \pm 0.39$ & $0.159 \pm 0.037$ & $-1.383 \pm 0.158$ & $-1.188 \pm 0.159$ \\
\hline $2020 \mathrm{dpw}$ & 0.320 & 1424 & $31.68 \pm 0.17$ & $58904.8 \pm 0.7$ & $<-16.945$ & $-16.75 \pm 0.42$ & $0.078 \pm 0.004$ & $-1.368 \pm 0.150$ & $-1.257 \pm 0.174$ \\
\hline $2020 \mathrm{dvt}$ & 0.013 & 4200 & $33.82 \pm 0.18$ & $58908.5 \pm 2.0$ & -17.398 & $-16.91 \pm 0.41$ & $0.217 \pm 0.023$ & $-1.628 \pm 0.150$ & $-1.719 \pm 0.168$ \\
\hline 2020ekk & 0.041 & 4273 & $33.80 \pm 0.16$ & $58918.7 \pm 0.6$ & -17.905 & $-17.22 \pm 0.40$ & $0.156 \pm 0.020$ & $-1.452 \pm 0.150$ & $-1.666 \pm 0.165$ \\
\hline $2020 \mathrm{gcv}$ & 0.031 & 8513 & $35.27 \pm 0.10$ & $58934.5 \pm 6.5$ & $<-17.629$ & $-17.32 \pm 0.40$ & $0.090 \pm 0.014$ & $-1.267 \pm 0.152$ & $-1.171 \pm 0.159$ \\
\hline 2020 ifc & 0.173 & 4683 & $33.79 \pm 0.15$ & $58930.5 \pm 5.7$ & -15.851 & $-15.62 \pm 0.41$ & $0.133 \pm 0.139$ & $-1.698 \pm 0.290$ & $-1.760 \pm 0.167$ \\
\hline 2020nja & 0.026 & 5656 & $34.29 \pm 0.15$ & $59015.2 \pm 4.9$ & $<-16.702$ & $-16.50 \pm 0.40$ & $0.074 \pm 0.008$ & $-1.412 \pm 0.148$ & $-1.519 \pm 0.166$ \\
\hline 2020umi & 0.058 & 5610 & $34.43 \pm 0.15$ & $59119.5 \pm 1.0$ & -17.860 & $-17.00 \pm 0.40$ & $0.067 \pm 0.018$ & $-1.253 \pm 0.163$ & $-1.214 \pm 0.169$ \\
\hline
\end{tabular}

